\title{
REGULARITY THEORY FOR 2-DIMENSIONAL ALMOST MINIMAL CURRENTS III: BLOWUP
}

\author{
CAMILLO DE LELLIS, EMANUELE SPADARO AND LUCA SPOLAOR
}

\begin{abstract}
We analyze the asymptotic behavior of a 2-dimensional integral current which is almost minimizing in a suitable sense at a singular point. Our analysis is the second half of an argument which shows the discreteness of the singular set for the following three classes of 2-dimensional currents: area minimizing in Riemannian manifolds, semicalibrated and spherical cross sections of 3 -dimensional area minimizing cones.
\end{abstract}

This paper is the fourth and last in a series of works aimed at establishing an optimal regularity theory for 2-dimensional integral currents which are almost minimizing in a suitable sense. Building upon the monumental work of Almgren [1], Chang in [3] established that 2-dimensional area-minimizing currents in Riemannian manifolds are classical minimal surfaces, namely they are regular (in the interior) except for a discrete set of branching singularities. The argument of Chang is however not entirely complete since a key starting point of his analysis, the existence of the so-called "branched center manifold", is only sketched in the appendix of [3] and requires the understanding (and a suitable modification) of the most involved portion of the monograph [1].

An alternative proof of Chang's theorem has been found by Rivière and Tian in [13] for the special case of $J$-holomorphic curves. Later on the approach of Rivière and Tian has been generalized by Bellettini and Rivière in [2] to handle a case which is not covered by [3], namely that of special Legendrian cycles in $\mathbb{S}^{5}$.

Meanwhile the first and second author revisited Almgren's theory giving a much shorter version of his program for proving that area-minimizing currents are regular up to a set of Hausdorff codimension 2, cf. [4, 5, 6, 7, 8]. In this note and its companion papers [10,9] we build upon the latter works in order to give a complete regularity theory which includes both the theorems of Chang and Bellettini-Rivière as special cases. In order to be more precise, we introduce the following terminology (cf. [11, Definition 0.3]).

Definition 0.1. Let $\Sigma \subset R^{m+n}$ be a $C^{2}$ submanifold and $U \subset \mathbb{R}^{m+n}$ an open set.

(a) An $m$-dimensional integral current $T$ with finite mass and $\operatorname{spt}(T) \subset \Sigma \cap U$ is areaminimizing in $\Sigma \cap U$ if $\mathbf{M}(T+\partial S) \geq \mathbf{M}(T)$ for any $m+$ 1-dimensional integral current $S$ with $\operatorname{spt}(S) \subset \subset \Sigma \cap U$.

(b) A semicalibration (in $\Sigma$ ) is a $C^{1} m$-form $\omega$ on $\Sigma$ such that $\left\|\omega_{x}\right\|_{c} \leq 1$ at every $x \in \Sigma$, where $\|\cdot\|_{c}$ denotes the comass norm on $\Lambda^{m} T_{x} \Sigma$. An $m$-dimensional integral current $T$ with $\operatorname{spt}(T) \subset \Sigma$ is semicalibrated by $\omega$ if $\omega_{x}(\vec{T})=1$ for $\|T\|$-a.e. $x$.

(c) An $m$-dimensional integral current $T$ supported in $\partial \mathbf{B}_{\bar{R}}(p) \subset \mathbb{R}^{m+n}$ is a spherical cross-section of an area-minimizing cone if $p \times T$ is area-minimizing. 
In what follows, given an integer rectifiable current $T$, we denote by $\operatorname{Reg}(T)$ the subset of $\operatorname{spt}(T) \backslash \operatorname{spt}(\partial T)$ consisting of those points $x$ for which there is a neighborhood $U$ such that $T\llcorner U$ is a (costant multiple of) a regular submanifold. Correspondingly, $\operatorname{Sing}(T)$ is the set $\operatorname{spt}(T) \backslash(\operatorname{spt}(\partial T) \cup \operatorname{Reg}(T))$. Observe that $\operatorname{Reg}(T)$ is relatively open in $\operatorname{spt}(T) \backslash \operatorname{spt}(\partial T)$ and thus $\operatorname{Sing}(T)$ is relatively closed. The main result of this and the works $[10,9]$ is then the following

Theorem 0.2. Let $m=2$ and $T$ be as in (a), (b) or (c) of Definition 0.1. Assume in addition that $\Sigma$ is of class $C^{3, \varepsilon_{0}}$ (in case (a) and (b)) and $\omega$ of class $C^{2, \varepsilon_{0}}$ (in case (b)) for some positive $\varepsilon_{0}$. Then $\operatorname{Sing}(T)$ is discrete.

Clearly Chang's result is covered by case (a). As for the case of special Lagrangian cycles considered by Bellettini and Rivière in [2] observe that they form a special subclass of both (b) and (c). Indeed these cycles arise as spherical cross-sections of 3-dimensional special lagrangian cones: as such they are then spherical cross sections of area-minimizing cones but they are also semicalibrated by a specific smooth form on $\mathbb{S}^{5}$.

Following the Almgren-Chang program, Theorem 0.2 will be established through a suitable "blow-up argument": this argument is presented here but requires several tools. The first important tool is the theory of multiple-valued functions, for which we will use the results and terminology of the papers $[4,5]$. The second tool is a suitable approximation result for area-minimizing currents with graphs of multiple valued functions, which for the case at hand has been established in the preceding note [10]. The last tool is the so-called "branched center manifold": this has been constructed in the paper [9]. We note in passing that all our arguments use heavily the uniqueness of tangent cones for $T$. This result is a, by now classical, theorem of White for area-minimizing 2-dimensional currents in the euclidean space, cf. [15]. Chang extended it to case (a) in the appendix of [3], whereas Pumberger and Rivière covered case (b) in [12]. A general derivation of these results for a wide class of almost minimizers has been given in [11]: the theorems in there cover, in particular, all the cases of Definition 0.1.

The proof of Theorem 0.2 is based, as in [3], on an induction statement, cf. Theorem 1.8 below. Although Theorem 1.8 is already stated in [9], we will recall it in the next section, where we also show how it implies Theorem 0.2. This and the previous paper [9] can be hence thought as the two halves in the proof of Theorem 1.8. After introducing some terminology, in Section 2 we will state the first half in Theorem 2.6 (which is proved in [9]) and the second half in Theorem 2.8, and we will then show how they fit together to prove Theorem 1.8. The remaining sections (the biggest portion of this note) are then dedicated to prove Theorem 2.8 .

Acknowledgments The research of Camillo De Lellis and Luca Spolaor has been supported by the ERC grant RAM (Regularity for Area Minimizing currents), ERC 306247.

\section{PREliminaries AND The MAin IndUCtion STATEMENT}

1.1. Basic notation and first main assumptions. For the notation concerning submanifolds $\Sigma \subset \mathbb{R}^{2+n}$ we refer to $[6$, Section 1$]$. With $\mathbf{B}_{r}(p)$ and $B_{r}(x)$ we denote, respectively, the open ball with radius $r$ and center $p$ in $\mathbb{R}^{2+n}$ and the open ball with radius $r$ 
and center $x$ in $\mathbb{R}^{2} . \mathbf{C}_{r}(p)$ and $\mathbf{C}_{r}(x)$ will always denote the cylinder $B_{r}(x) \times \mathbb{R}^{n}$, where $p=(x, y) \in \mathbb{R}^{2} \times \mathbb{R}^{n}$. We will often need to consider cylinders whose bases are parallel to other 2-dimensional planes, as well as balls in $m$-dimensional affine planes. We then introduce the notation $B_{r}(p, \pi)$ for $\mathbf{B}_{r}(p) \cap(p+\pi)$ and $\mathbf{C}_{r}(p, \pi)$ for $B_{r}(p, \pi)+\pi^{\perp}$. $e_{i}$ will denote the unit vectors in the standard basis, $\pi_{0}$ the (oriented) plane $\mathbb{R}^{2} \times\{0\}$ and $\vec{\pi}_{0}$ the 2 -vector $e_{1} \wedge e_{2}$ orienting it. Given an $m$-dimensional plane $\pi$, we denote by $\mathbf{p}_{\pi}$ and $\mathbf{p}_{\pi}^{\perp}$ the orthogonal projections onto, respectively, $\pi$ and its orthogonal complement $\pi^{\perp}$. For what concerns integral currents we use the definitions and the notation of [14]. Since $\pi$ is used recurrently for 2-dimensional planes, the 2-dimensional area of the unit circle in $\mathbb{R}^{2}$ will be denoted by $\omega_{2}$.

By [10, Lemma 1.1] in case (b) we can assume, without loss of generality, that the ambient manifold $\Sigma$ coincides with the euclidean space $\mathbb{R}^{2+n}$. In the rest of the paper we will therefore always make the following

Assumption 1.1. $T$ is an integral current of dimension 2 with bounded support and it satisfies one of the three conditions (a), (b) or (c) in Definition 0.1. Moreover

- In case (a), $\Sigma \subset \mathbb{R}^{2+n}$ is a $C^{3, \varepsilon_{0}}$ submanifold of dimension $2+\bar{n}=2+n-l$, which is the graph of an entire function $\Psi: \mathbb{R}^{2+\bar{n}} \rightarrow \mathbb{R}^{l}$ and satisfies the bounds

$$
\|D \Psi\|_{0} \leq c_{0} \quad \text { and } \quad \mathbf{A}:=\left\|A_{\Sigma}\right\|_{0} \leq c_{0},
$$

where $c_{0}$ is a positive (small) dimensional constant and $\left.\varepsilon_{0} \in\right] 0,1[$.

- In case (b) we assume that $\Sigma=\mathbb{R}^{2+n}$ and that the semicalibrating form $\omega$ is $C^{2, \varepsilon_{0}}$.

- In case (c) we assume that $T$ is supported in $\Sigma=\partial \mathbf{B}_{R}\left(p_{0}\right)$ for some $p_{0}$ with $\left|p_{0}\right|=R$, so that $0 \in \partial \mathbf{B}_{R}\left(p_{0}\right)$. We assume also that $T_{0} \partial \mathbf{B}_{R}\left(p_{0}\right)$ is $\mathbb{R}^{2+n-1}$ (namely $p_{0}=\left(0, \ldots, 0, \pm\left|p_{0}\right|\right)$ and we let $\Psi: \mathbb{R}^{2+n-1} \rightarrow \mathbb{R}$ be a smooth extension to the whole space of the function which describes $\Sigma$ in $\mathbf{B}_{2}(0)$. We assume then that (1.1) holds, which is equivalent to the requirement that $R^{-1}$ be sufficiently small.

In addition, since the conclusion of Theorem 0.2 is local, by [11, Proposition 0.4] we can also assume to be always in the following situation.

Assumption 1.2. In addition to Assumption 1.1 we assume the following:

(i) $\partial T\left\llcorner\mathbf{C}_{2}\left(0, \pi_{0}\right)=0\right.$;

(ii) $0 \in \operatorname{spt}(T)$ and the tangent cone at 0 is given by $\Theta(T, 0) \llbracket \pi_{0} \rrbracket$ where $\Theta(T, 0) \in$ $\mathbb{N} \backslash\{0\}$

(iii) $T$ is irreducible in any neighborhood $U$ of 0 in the following sense: it is not possible to find $S, Z$ non-zero integer rectifiable currents in $U$ with $\partial S=\partial Z=0$ (in $U$ ), $T=S+Z$ and $\operatorname{spt}(S) \cap \operatorname{spt}(Z)=\{0\}$.

In order to justify point (iii), observe that we can argue as in the proof of [11, Theorem 3.1]: assuming that in a certain neighborhood $U$ there is a decomposition $T=S+Z$ as above, it follows from [11, Proposition 2.2] that both $S$ and $Z$ fall in one of the classes of Definition 0.1. In turn this implies that $\Theta(S, 0), \Theta(Z, 0) \in \mathbb{N} \backslash\{0\}$ and thus $\Theta(S, 0)<$ $\Theta(T, 0)$. We can then replace $T$ with either $S$ or $Z$. Let $T_{1}=S$ and argue similarly if it is not irreducibile: obviously we can apply one more time the argument above and find a 
$T_{2}$ which satisfies all the requirements and has $0<\Theta\left(T_{2}, 0\right)<\Theta\left(T_{1}, 0\right)$. This process must stop after at most $N=\Theta(T, 0)$ steps: the final current is then necessarily irreducible.

1.2. Branching model. We next introduce an object which will play a key role in the rest of our work, because it is the basic local model of the singular behavior of a 2-dimensional area-minimizing current: for each positive natural number $Q$ we will denote by $\mathfrak{B}_{Q, \rho}$ the flat Riemann surface which is a disk with a conical singularity, in the origin, of angle $2 \pi Q$ and radius $\rho>0$. More precisely we have

Definition 1.3. $\mathfrak{B}_{Q, \rho}$ is topologically an open 2-dimensional disk, which we identify with the topological space $\left\{(z, w) \in \mathbb{C}^{2}: w^{Q}=z,|z|<\rho\right\}$. For each $\left(z_{0}, w_{0}\right) \neq 0$ in $\mathfrak{B}_{Q, \rho}$ we consider the connected component $\mathfrak{D}\left(z_{0}, w_{0}\right)$ of $\mathfrak{B}_{Q, \rho} \cap\left\{(z, w):\left|z-z_{0}\right|<\left|z_{0}\right| / 2\right\}$ which contains $\left(z_{0}, w_{0}\right)$. We then consider the smooth manifold given by the atlas

$$
\left.\left\{(\mathfrak{D}(z, w)),\left(x_{1}, x_{2}\right)\right):(z, w) \in \mathfrak{B}_{Q, \rho} \backslash\{0\}\right\},
$$

where $\left(x_{1}, x_{2}\right)$ is the function which gives the real and imaginary part of the first complex coordinate of a generic point of $\mathfrak{B}_{Q, \rho}$. On such smooth manifold we consider the following flat Riemannian metric: on each $\mathfrak{D}(z, w)$ with the chart $\left(x_{1}, x_{2}\right)$ the metric tensor is the usual euclidean one $d x_{1}^{2}+d x_{2}^{2}$. Such metric will be called the canonical flat metric and denoted by $e_{Q}$. The coordinates $\left(x_{1}, x_{2}\right)=z$ will be called standard flat coordinates.

When $Q=1$ we can extend smoothly the metric tensor to the origin and we obtain the usual euclidean 2-dimensional disk. For $Q>1$ the metric tensor does not extend smoothly to 0 , but we can nonetheless complete the induced geodesic distance on $\mathfrak{B}_{Q, \rho}$ in a neighborhood of 0 : for $(z, w) \neq 0$ the distance to the origin will then correspond to $|z|$. The resulting metric space is a well-known object in the literature, namely a flat Riemann surface with an isolated conical singularity at the origin (see for instance [16]). Note that for each $z_{0}$ and $0<r \leq \min \left\{\left|z_{0}\right|, \rho-\left|z_{0}\right|\right\}$ the set $\mathfrak{B}_{Q, \rho} \cap\left\{\left|z-z_{0}\right|<r\right\}$ consists then of $Q$ nonintersecting 2-dimensional disks, each of which is a geodesic ball of $\mathfrak{B}_{Q, \rho}$ with radius $r$ and center $\left(z_{0}, w_{i}\right)$ for some $w_{i} \in \mathbb{C}$ with $w_{i}^{Q}=z_{0}$. We then denote each of them by $B_{r}\left(z_{0}, w_{i}\right)$ and treat it as a standard disk in the euclidean 2-dimensional plane (which is correct from the metric point of view). We use however the same notation for the distance disk $B_{r}(0)$, namely for the set $\{(z, w):|z|<r\}$, although the latter is not isometric to the standard euclidean disk. Since this might be create some ambiguity, we will use the specification $\mathbb{R}^{2} \supset B_{r}(0)$ when referring to the standard disk in $\mathbb{R}^{2}$.

1.3. Admissible $Q$-branchings. When one of (or both) the parameters $Q$ and $\rho$ are clear from the context, the corresponding subscript (or both) will be omitted. We will consider repeatedly functions $u$ defined on $\mathfrak{B}$. We will always treat each point of $\mathfrak{B}$ as an element of $\mathbb{C}^{2}$, mostly using $z$ and $w$ for the horizontal and vertical complex coordinates. Often $\mathbb{C}$ will be identified with $\mathbb{R}^{2}$ and thus the coordinate $z$ will be treated as a two-dimensional real vector, avoiding the more cumbersome notation $\left(x_{1}, x_{2}\right)$.

Definition 1.4 (Q-branchings). Let $\alpha \in] 0,1[, b>1, Q \in \mathbb{N} \backslash\{0\}$ and $n \in \mathbb{N} \backslash\{0\}$. An admissible $\alpha$-smooth and $b$-separated $Q$-branching in $\mathbb{R}^{2+n}$ (shortly a $Q$-branching) is the 
graph

$$
\operatorname{Gr}(u):=\left\{(z, u(z, w)):(z, w) \in \mathfrak{B}_{Q, 2 \rho}\right\} \subset \mathbb{R}^{2+n}
$$

of a map $u: \mathfrak{B}_{Q, 2 \rho} \rightarrow \mathbb{R}^{n}$ satisfying the following assumptions. For some constants $C_{i}>0$ we have

- $u$ is continuous, $u \in C^{3, \alpha}$ on $\mathfrak{B}_{Q, \rho} \backslash\{0\}$ and $u(0)=0$;

- $\left|D^{j} u(z, w)\right| \leq C_{i}|z|^{1-j+\alpha} \forall(z, w) \neq 0$ and $j \in\{0,1,2,3\}$;

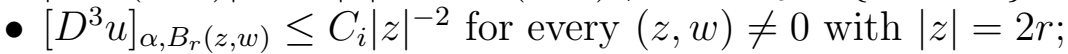

- If $Q>1$, then there is a positive constant $\left.c_{s} \in\right] 0,1[$ such that

$$
\min \left\{\left|u(z, w)-u\left(z, w^{\prime}\right)\right|: w \neq w^{\prime}\right\} \geq 4 c_{s}|z|^{b} \quad \text { for all }(z, w) \neq 0 .
$$

The map $\boldsymbol{\Phi}(z, w):=(z, u(z, w))$ will be called the graphical parametrization of the $Q$ branching.

Any $Q$-branching as in the Definition above is an immersed disk in $\mathbb{R}^{2+n}$ and can be given a natural structure as integer rectifiable current, which will be denoted by $\mathbf{G}_{u}$. For $Q=1$ a map $u$ as in Definition 1.4 is a (single valued) $C^{1, \alpha}$ map $u: \mathbb{R}^{2} \supset B_{2 \rho}(0) \rightarrow \mathbb{R}^{n}$. Although the term branching is not appropriate in this case, the advantage of our setup is that $Q=1$ will not be a special case in the induction statement of Theorem 1.8 below. Observe that for $Q>1$ the map $u$ can be thought as a $Q$-valued map $u: \mathbb{R}^{2} \supset B_{\rho}(0) \rightarrow \mathcal{A}_{Q}\left(\mathbb{R}^{n}\right)$, setting $u(z)=\sum_{\left(z, w_{i}\right) \in \mathfrak{B}} \llbracket u\left(z, w_{i}\right) \rrbracket$ for $z \neq 0$ and $u(0)=Q \llbracket 0 \rrbracket$. The notation $\operatorname{Gr}(u)$ and $\mathbf{G}_{u}$ is then coherent with the corresponding objects defined in [5] for general $Q$-valued maps.

1.4. The inductive statement. Before coming to the key inductive statement, we need to introduce some more terminology.

Definition 1.5 (Horned Neighborhood). Let $\operatorname{Gr}(u)$ be a $b$-separated $Q$-branching. For every $a>b$ we define the horned neighborhood $\mathbf{V}_{u, a}$ of $\operatorname{Gr}(u)$ to be

$$
\mathbf{V}_{u, a}:=\left\{(x, y) \in \mathbb{R}^{2} \times \mathbb{R}^{n}: \exists(x, w) \in \mathfrak{B}_{Q, 2 \rho} \text { with }|y-u(x, w)|<c_{s}|x|^{a}\right\},
$$

where $c_{s}$ is the constant in (1.3).

Definition 1.6 (Excess). Given an $m$-dimensional current $T$ in $\mathbb{R}^{m+n}$ with finite mass, its excess in the ball $\mathbf{B}_{r}(x)$ and in the cylinder $\mathbf{C}_{r}\left(p, \pi^{\prime}\right)$ with respect to the $m$-plane $\pi$ are

$$
\begin{aligned}
\mathbf{E}\left(T, \mathbf{B}_{r}(p), \pi\right) & :=\left(2 \omega_{m} r^{m}\right)^{-1} \int_{\mathbf{B}_{r}(p)}|\vec{T}-\vec{\pi}|^{2} d\|T\| \\
\mathbf{E}\left(T, \mathbf{C}_{r}\left(p, \pi^{\prime}\right), \pi\right) & :=\left(2 \omega_{m} r^{m}\right)^{-1} \int_{\mathbf{C}_{r}\left(p, \pi^{\prime}\right)}|\vec{T}-\vec{\pi}|^{2} d\|T\| .
\end{aligned}
$$

For cylinders we omit the third entry when $\pi=\pi^{\prime}$, i.e. $\mathbf{E}\left(T, \mathbf{C}_{r}(p, \pi)\right):=\mathbf{E}\left(T, \mathbf{C}_{r}(p, \pi), \pi\right)$. In order to define the spherical excess we consider $T$ as in Assumption 1.1 and we say that $\pi$ optimizes the excess of $T$ in a ball $\mathbf{B}_{r}(x)$ if

- In case (b)

$$
\mathbf{E}\left(T, \mathbf{B}_{r}(x)\right):=\min _{\tau} \mathbf{E}\left(T, \mathbf{B}_{r}(x), \tau\right)=\mathbf{E}\left(T, \mathbf{B}_{r}(x), \pi\right) ;
$$


- In case (a) and (c) $\pi \subset T_{x} \Sigma$ and

$$
\mathbf{E}\left(T, \mathbf{B}_{r}(x)\right):=\min _{\tau \subset T_{x} \Sigma} \mathbf{E}\left(T, \mathbf{B}_{r}(x), \tau\right)=\mathbf{E}\left(T, \mathbf{B}_{r}(x), \pi\right) .
$$

Note in particular that, in case (a) and (c), $\mathbf{E}\left(T, \mathbf{B}_{r}(x)\right)$ differs from the quantity defined in [8, Definition 1.1], where, although $\Sigma$ does not coincide with the ambient euclidean space, $\tau$ is allowed to vary among all planes, as in case (b). Thus a notation more consistent with that of [8] would be, in case (a) and (c), $\mathbf{E}^{\Sigma}\left(T, \mathbf{B}_{r}(x)\right)$. However, the difference is a minor one and we prefer to keep our notation simpler.

Our main induction assumption is then the following

Assumption 1.7 (Inductive Assumption). $T$ is as in Assumption 1.1 and 1.2. For some constants $\bar{Q} \in \mathbb{N} \backslash\{0\}$ and $0<\alpha<\frac{1}{2 Q}$ there is an $\alpha$-admissible $\bar{Q}$-branching $\operatorname{Gr}(u)$ with $u: \mathfrak{B}_{\bar{Q}, 2} \rightarrow \mathbb{R}^{n}$ such that

(Sep) If $\bar{Q}>1, u$ is $b$-separated for some $b>1$; a choice of some $b>1$ is fixed also in the case $\bar{Q}=1$, although in this case the separation condition is empty.

(Hor) $\operatorname{spt}(T) \subset \mathbf{V}_{u, a} \cup\{0\}$ for some $a>b$;

(Dec) There exist $\gamma>0$ and a $C_{i}>0$ with the following property. Let $p=\left(x_{0}, y_{0}\right) \in$ $\operatorname{spt}(T) \cap \mathbf{C}_{\sqrt{2}}(0)$ and $4 d:=\left|x_{0}\right|>0$, let $V$ be the connected component of $\mathbf{V}_{u, a} \cap$ $\left\{(x, y):\left|x-x_{0}\right|<d\right\}$ containing $p$ and let $\pi(p)$ be the plane tangent to $\operatorname{Gr}(u)$ at the only point of the form $\left(x_{0}, u\left(x_{0}, w_{i}\right)\right)$ which is contained in $V$. Then

$$
\mathbf{E}\left(T\left\llcorner V, \mathbf{B}_{\sigma}(p), \pi(p)\right) \leq C_{i}^{2} d^{2 \gamma-2} \sigma^{2} \quad \forall \sigma \in\left[\frac{1}{2} d^{(b+1) / 2}, d\right] .\right.
$$

The main inductive step is then the following theorem, where we denote by $T_{p, r}$ the rescaled current $\left(\iota_{p, r}\right)_{\sharp} T$, through the map $\iota_{p, r}(q):=(q-p) / r$.

Theorem 1.8 (Inductive statement). Let $T$ be as in Assumption 1.7 for some $\bar{Q}=Q_{0}$. Then,

(a) either $T$ is, in a neighborhood of 0, a $Q$ multiple of a $\bar{Q}$-branching $\operatorname{Gr}(v)$;

(b) or there are $r>0$ and $Q_{1}>Q$ such that $T_{0, r}$ satisfies Assumption 1.7 with $\bar{Q}=Q_{1}$.

Theorem 0.2 follows then easily from Theorem 1.8 and [11].

1.5. Proof of Theorem 0.2. As already mentioned, without loss of generality we can assume that Assumption 1.1 holds, cf. [11, Lemma 1.1] (the bounds on $\mathbf{A}$ and $\Psi$ can be achieved by a simple scaling argument). Fix now a point $p$ in $\operatorname{spt}(T) \backslash \operatorname{spt}(\partial T)$. Our aim is to show that $T$ is regular in a punctured neighborhood of $p$. Without loss of generality we can assume that $p$ is the origin. Upon suitably decomposing $T$ in some neighborhood of 0 we can easily assume that (I) in Assumption 1.7 holds, cf. the argument of Step 4 in the proof of [11, Theorem 3.1]. Thus, upon suitably rescaling and rotating $T$ we can assume that $\pi_{0}$ is the unique tangent cone to $T$ at 0 , cf. [11, Theorem 3.1]. In fact, by [11, Theorem 3.1] $T$ satisfies Assumption 1.7 with $\bar{Q}=1$ : it suffices to chose $u \equiv 0$ as admissible smooth branching. If $T$ were not regular in any punctured neighborhood of 0 , we could then apply Theorem 1.8 inductively to find a sequence of rescalings $T_{0, \rho_{j}}$ with $\rho_{j} \downarrow 0$ which satisfy Assumption 1.7 with $\bar{Q}=Q_{j}$ for some strictly increasing sequence of 
integers. It is however elementary that the density $\Theta(0, T)$ bounds $Q_{j}$ from above, which is a contradiction.

\section{The BRANCHED CENTER MANifold AND THE BLOW-UP THEOREM}

From now on we fix $T$ satisfying Assumption 1.7. Observe that, without loss of generality, we are always free to rescale homothetically our current $T$ with a factor larger than 1 and ignore whatever portion falls outside $\mathbf{C}_{2}(0)$. We will do this several times, with factors which will be assumed to be sufficiently large. Hence, if we can prove that something holds in a sufficiently small neighborhood of 0 , then we can assume, withouth loss of generality, that it holds on $\mathbf{C}_{2}$. For this reason we can assume that the constants $C_{i}$ in Definition 1.4 and Assumption 1.7 is as small as we want. In turns this implies that there is a well-defined orthogonal projection $\mathbf{P}: \mathbf{V}_{u, a} \cap \mathbf{C}_{1} \rightarrow \operatorname{Gr}(u) \cap \mathbf{C}_{2}$, which is a $C^{2, \alpha}$ map. We next recall [9, Lemma 2.1]:

Lemma 2.1. Let $T$ and $u$ be as in Assumption 1.7 for some $\bar{Q}$. Then the nearest point projection $\mathbf{P}: \mathbf{V}_{u, a} \cap \mathbf{C}_{1} \rightarrow \operatorname{Gr}(u)$ is a well-defined $C^{2, \alpha}$ map. In addition there is $Q \in$ $\mathbb{N} \backslash\{0\}$ such that $\Theta(0, T)=Q \bar{Q}$ and the unique tangent cone to $T$ at 0 is $Q \bar{Q} \llbracket \pi_{0} \rrbracket$. Finally, after possibly rescaling $T, \Theta(p, T) \leq Q$ for every $p \in \mathbf{C}_{2} \backslash\{0\}$ and, for every $x \in B_{2}(0)$, each connected component of $\left(x \times \mathbb{R}^{n}\right) \cap \mathbf{V}_{u, a}$ contains at least one point of $\operatorname{spt}(T)$.

Since we will assume during the rest of the paper that the above discussion applies, we summarize the relevant conclusions in the following

Assumption 2.2. $T$ satisfies Assumption 1.7 for some $\bar{Q}$ and with $C_{i}$ sufficiently small. $Q \geq 1$ is an integer, $\Theta(0, T)=Q \bar{Q}$ and $\Theta(p, T) \leq Q$ for all $p \in \mathbf{C}_{2} \backslash\{0\}$.

The overall plan to prove Theorem 1.8 is then the following:

(CM) We construct first a branched center manifold, i.e. a second admissible smooth branching $\varphi$ on $\mathfrak{B}_{\bar{Q}}$, and a corresponding $Q$-valued map $N$ defined on the normal bundle of $\operatorname{Gr}(\varphi)$, which approximates $T$ with a very high degree of accuracy (in particular more accurately than $u$ ) and whose average $\boldsymbol{\eta} \circ N$ is very small;

(BU) Assuming that alternative (a) in Theorem 1.8 does not hold, we study the asymptotic behavior of $N$ around 0 and use it to build a new admissible smooth branching $v$ on some $\mathfrak{B}_{k \bar{Q}}$ where $k \geq 2$ is a factor of $Q$ : this map will then be the one sought in alternative (b) of Theorem 1.8 and a suitable rescaling of $T$ will lie in a horned neighborhood of its graph.

The first part of the program is the one achieved in [9], whereas the second part will be completed in this note. Note that, when $Q=1$, from $(\mathrm{BU})$ we will conclude that alternative (a) necessarily holds: this will be a simple corollary of the general case, but we observe that it could also be proved resorting to the classical Allard's regularity theorem.

2.1. Smallness condition. In several occasions we will need that the ambient manifold $\Sigma$ is suitably flat and that the excess of the current $T$ is suitably small. This can, however, be easily achieved after scaling. More precisely we recall [9, Lemma 2.3]. 
Lemma 2.3. Let $T$ be as in the Assumptions 1.7 and 2.2. After possibly rescaling, rotating and modifying $\Sigma$ outside $\mathbf{C}_{2}(0)$ we can assume that, in case (a) and (c) of Definition 0.1,

(i) $\Sigma$ is a complete submanifold of $\mathbb{R}^{2+n}$;

(ii) $T_{0} \Sigma=\mathbb{R}^{2+\bar{n}} \times\{0\}$ and, $\forall p \in \Sigma, \Sigma$ is the graph of a $C^{3, \varepsilon_{0}}$ map $\Psi_{p}: T_{p} \Sigma \rightarrow\left(T_{p} \Sigma\right)^{\perp}$. Under these assumptions, we denote by $\boldsymbol{c}$ and $\boldsymbol{m}_{0}$ the following quantities

$$
\begin{array}{ll}
\boldsymbol{c}:=\sup \left\{\left\|D \Psi_{p}\right\|_{C^{2, \varepsilon_{0}}}: p \in \Sigma\right\} & \text { in the cases (a) and }(c) \text { of Definition } 0.1 \\
\boldsymbol{c}:=\|d \omega\|_{C^{1, \varepsilon_{0}}} & \text { in case }(b) \text { of Definition } 0.1 \\
\boldsymbol{m}_{0}:=\max \left\{\boldsymbol{c}^{2}, \mathbf{E}\left(T, \mathbf{C}_{2}, \pi_{0}\right), C_{i}^{2}, c_{s}^{2}\right\} &
\end{array}
$$

where $C_{i}$ and $c_{s}$ are the constants appearing in Definition 1.4 and Assumption 1.7. Then, for any $\varepsilon_{2}>0$, after possibly rescaling the current by a large factor, we can assume

$$
\boldsymbol{m}_{0} \leq \varepsilon_{2} .
$$

In order to carry on the plan outlined in the previous subsection, it is convenient to use a different parametrization of $Q$-branchings. If we remove the origin, any admissible $Q$-branching is a Riemannian submanifold of $\mathbb{R}^{2+n} \backslash\{0\}$ : this gives a Riemannian tensor $g:=\boldsymbol{\Phi}^{\sharp} e$ (where $e$ denotes the euclidean metric on $\mathbb{R}^{2+n}$ ) on the puntured disk $\mathfrak{B}_{Q, 2 \rho} \backslash\{0\}$. Note that in $(z, w)$ the difference between the metric tensor $g$ and the canonical flat metric $e_{Q}$ can be estimated by (a constant times) $|z|^{2 \alpha}$ : thus, as it happens for the canonical flat metric $e_{Q}$, when $Q>1$ it is not possible to extend the metric $g$ to the origin. However, using well-known arguments in differential geometry, we can find a conformal map from $\mathfrak{B}_{Q, r}$ onto a neighborhood of 0 which maps the conical singularity of $\mathfrak{B}_{Q, r}$ in the conical singularity of the $Q$-branching. In fact, we need the following accurate estimates for such a map, cf. [9, Proposition 2.4]:

Proposition 2.4 (Conformal parametrization). Given an admissible b-separated $\alpha$-smooth $Q$-branching $\operatorname{Gr}(u)$ with $\alpha<1 /(2 Q)$ there exist a constant $C_{0}(Q, \alpha)>0$, a radius $r>0$ and functions $\boldsymbol{\Psi}: \mathfrak{B}_{Q, r} \rightarrow \operatorname{Gr}(u)$ and $\lambda: \mathfrak{B}_{Q, r} \rightarrow \mathbb{R}_{+}$such that

(i) $\boldsymbol{\Psi}$ is a homeomorphism of $\mathfrak{B}_{Q, r}$ with a neighborhood of 0 in $\operatorname{Gr}(u)$;

(ii) $\boldsymbol{\Psi} \in C^{3, \alpha}\left(\mathfrak{B}_{Q, r} \backslash\{0\}\right)$, with the estimates

$$
\begin{aligned}
\left|D^{l}(\Psi(z, w)-(z, 0))\right| & \leq C_{0} C_{i}|z|^{1+\alpha-l} \quad \text { for } l=0, \ldots, 3, z \neq 0, \\
{\left[D^{3} \boldsymbol{\Psi}\right]_{\alpha, B_{r}(z, w)} } & \leq C_{0} C_{i}|z|^{-2} \quad \text { for } z \neq 0 \text { and } r=|z| / 2 ;
\end{aligned}
$$

(iii) $\boldsymbol{\Psi}$ is a conformal map with conformal factor $\lambda$, namely, if we denote by $e$ the ambient euclidean metric in $\mathbb{R}^{2+n}$ and by $e_{Q}$ the canonical euclidean metric of $\mathfrak{B}_{Q, r}$,

$$
g:=\Psi^{\sharp} e=\lambda e_{Q} \quad \text { on } \mathfrak{B}_{Q, r} \backslash\{0\} .
$$

(iv) The conformal factor $\lambda$ satisfies

$$
\begin{aligned}
\left|D^{l}(\lambda-1)(z, w)\right| & \leq C_{0} C_{i}|z|^{2 \alpha-l} & & \text { for } l=0,1, \ldots, 2 \\
{\left[D^{2} \lambda\right]_{\alpha, B_{r}(z, w)} } & \leq C_{0} C_{i}|z|^{\alpha-2} & \text { for } z & \neq 0 \text { and } r=|z| / 2 .
\end{aligned}
$$


Definition 2.5. A map $\Psi$ as in Proposition 2.4 will be called a conformal parametrization of an admissible $Q$-branching.

2.2. The center manifold and the approximation. We are now ready to state the two "halves" of Theorem 1.8. The first one is [9, Theorem 2.6], which we recall here for the reader's convenienve.

Theorem 2.6 (Center Manifold Approximation). Let $T$ be as in Assumptions 1.7 and 2.2. Then there exist $\eta_{0}, \gamma_{0}, r_{0}, C>0, b>1$, an admissible b-separated $\gamma_{0}$-smooth $\bar{Q}$ branching $\mathcal{M}$, a corresponding conformal parametrization $\Psi: \mathfrak{B}_{\bar{Q}, 2} \rightarrow \mathcal{M}$ and a $Q$-valued map $\mathcal{N}: \mathfrak{B}_{\bar{Q}, 2} \rightarrow \mathcal{A}_{Q}\left(\mathbb{R}^{2+n}\right)$ with the following properties:

(i) $\bar{Q} Q=\Theta(T, 0)$ and

$$
\begin{aligned}
|D(\boldsymbol{\Psi}(z, w)-(z, 0))| & \leq C \boldsymbol{m}_{0}^{1 / 2}|z|^{\gamma_{0}} \\
\left|D^{2} \boldsymbol{\Psi}(z, w)\right|+|z|^{-1}\left|D^{3} \boldsymbol{\Psi}(z, w)\right| & \leq C \boldsymbol{m}_{0}^{1 / 2}|z|^{\gamma_{0}-1}
\end{aligned}
$$

in particular, if we denote by $A_{\mathcal{M}}$ the second fundamental form of $\mathcal{M} \backslash\{0\}$,

$$
\left|A_{\mathcal{M}}(\mathbf{\Psi}(z, w))\right|+|z|^{-1}\left|D_{\mathcal{M}} A_{\mathcal{M}}(\mathbf{\Psi}(z, w))\right| \leq C \boldsymbol{m}_{0}^{1 / 2}|z|^{\gamma_{0}-1} .
$$

(ii) $\mathcal{N}_{i}(z, w)$ is orthogonal to the tangent plane, at $\boldsymbol{\Psi}(z, w)$, to $\mathcal{M}$.

(iii) If we define $S:=T_{0, r_{0}}$, then $\operatorname{spt}(S) \cap \mathbf{C}_{1} \backslash\{0\}$ is contained in a suitable horned neighborhood of the $\bar{Q}$-branching, where the orthogonal projection $\mathbf{P}$ onto it is welldefined. Moreover, for every $r \in] 0,1[$ we have

$$
\left\|\left.\mathcal{N}\right|_{B_{r}}\right\|_{0}+\sup _{p \in \operatorname{spt}(S) \cap \mathbf{P}^{-1}\left(\mathbf{\Psi}\left(B_{r}\right)\right)}|p-\mathbf{P}(p)| \leq C \boldsymbol{m}_{0}^{1 / 4} r^{1+\gamma_{0} / 2} .
$$

(iv) If we define

$$
\begin{gathered}
\mathbf{D}(r):=\int_{B_{r}}|D \mathcal{N}|^{2} \quad \text { and } \quad \mathbf{H}(r):=\int_{\partial B_{r}}|\mathcal{N}|^{2}, \\
\mathbf{F}(r):=\int_{0}^{r} \frac{\mathbf{H}(t)}{t^{2-\gamma_{0}}} d t \quad \text { and } \quad \mathbf{\Lambda}(r):=\mathbf{D}(r)+\mathbf{F}(r),
\end{gathered}
$$

then the following estimates hold for every $r \in] 0,1[$ :

$$
\begin{aligned}
\operatorname{Lip}\left(\left.\mathcal{N}\right|_{B_{r}}\right) & \leq C \min \left\{\boldsymbol{\Lambda}^{\eta_{0}}(r), \boldsymbol{m}_{0}^{\eta_{0}} r^{\eta_{0}}\right\} \\
\boldsymbol{m}_{0}^{\eta_{0}} \int_{B_{r}}|z|^{\gamma_{0}-1}|\boldsymbol{\eta} \circ \mathcal{N}(z, w)| & \leq C \boldsymbol{\Lambda}^{\eta_{0}}(r) \mathbf{D}(r)+C \mathbf{F}(r) .
\end{aligned}
$$

(v) Finally, if we set

$$
\mathcal{F}(z, w):=\sum_{i} \llbracket \Psi(z, w)+\mathcal{N}_{i}(z, w) \rrbracket
$$

then

$$
\left\|S-\mathbf{T}_{\mathcal{F}}\right\|\left(\mathbf{P}^{-1}\left(\boldsymbol{\Psi}\left(B_{r}\right)\right)\right) \leq C \boldsymbol{\Lambda}^{\eta_{0}}(r) \mathbf{D}(r)+C \mathbf{F}(r)
$$


The second main step is the analysis of the asymptotic behaviour of $\mathcal{N}$ around the origin, which is the main focus of this paper.

Remark 2.7. In order to state it, we agree to define $W^{1,2}$ functions on $\mathfrak{B}$ in the following fashion: removing the origin 0 from $\mathfrak{B}$ we have a $C_{l o c}^{3}$ (flat) Riemannian manifold embedded in $\mathbb{R}^{4}$ and we can define $W^{1,2}$ maps on it following [4]. Alternatively we can use the conformal parametrization $\mathbf{W}: \mathbb{R}^{2}=\mathbb{C} \rightarrow \mathfrak{B}_{\bar{Q}}$ given by $\mathbf{W}(z)=\left(z^{\bar{Q}}, z\right)$ and agree that $u \in W^{1,2}\left(\mathfrak{B}_{\bar{Q}}\right)$ if $u \circ \mathbf{W}$ is in $W^{1,2}\left(\mathbb{R}^{2}\right)$. Since discrete sets have zero 2-capacity, it is immediate to verify that these two definitions are equivalent.

In a similar fashion, we will ignore the origin when integrating by parts Lipschitz vector fields, treating $\mathfrak{B}_{\bar{Q}}$ as a $C^{1}$ Riemannian manifold. It is straightforward to show that our assumption is correct, for instance removing a disk of radius $\varepsilon$ centered at the origin, integrating by parts and then letting $\varepsilon \downarrow 0$.

Theorem 2.8 (Blowup Analysis). Under the assumptions of Theorem 2.6, the following dichotomy holds:

(i) either there exists $s>0$ such that $\left.\mathcal{N}\right|_{B_{s}} \equiv Q \llbracket 0 \rrbracket$;

(ii) or there exist constants $I_{0}>1, a_{0}, \bar{r}, C>0$ and an $I_{0}$-homogeneous nontrivial Dir-minimizing function $g: \mathfrak{B}_{\bar{Q}} \rightarrow \mathcal{A}_{Q}\left(\mathbb{R}^{2+n}\right)$ such that

$-\boldsymbol{\eta} \circ g \equiv 0$,

$-g=\sum_{i} \llbracket\left(0, \bar{g}_{i}, 0\right) \rrbracket$, where $\bar{g}_{i}(x) \in \mathbb{R}^{\bar{n}}$ and $\left(0, \bar{g}_{i}(x), 0\right) \in \mathbb{R}^{2} \times \mathbb{R}^{\bar{n}} \times \mathbb{R}^{l}$,

- and the following estimates hold:

$$
\begin{aligned}
\mathcal{G}(\mathcal{N}(z, w), g(z, w)) & \leq C|z|^{I_{0}+a_{0}} & & \forall(z, w) \in \mathfrak{B}_{Q},|z|<\bar{r}, \\
\int_{B_{r+\rho} \backslash B_{r-\rho}}|D \mathcal{N}|^{2} & \leq C r^{2 I_{0}+a_{0}}+C r^{2 I_{0}-1} \rho & & \forall 4 \rho \leq r<1, \\
\mathbf{H}(r) & \leq C r \mathbf{D}(r) & & \forall r<1 .
\end{aligned}
$$

Remark 2.9. Note that, when $\bar{Q}=\Theta(T, 0)$, we necessarily have $Q=1$ and the second alternative is excluded. In particular we conclude that $T$ coincides with $\llbracket \mathcal{M} \rrbracket$ in a neighborhood of 0 and thus it is a regular submanifold in a punctured neighborhood of 0 .

Remark 2.10. By a simple dyadic argument it follows from (2.17) and (2.18) that

$$
\int_{B_{r}}|D \mathcal{N}|^{2} \leq C r^{2 I_{0}} \quad \text { and } \quad \mathbf{F}(r) \leq C r^{2 I_{0}+\gamma_{0}} \quad \forall r<1 .
$$

Below we show how to conclude Theorem 1.8 from Theorem 2.8. The remaining part of the paper is dedicated to the proof of the latter, which will be split in six sections each corresponding to one of the following steps.

(i) In Section 3 we will deduce an almost minimizing property for the map $\mathcal{N}$ in terms of its Dirichlet energy.

(ii) In Section 4 we will apply the almost minimizing property and compare the Dirichlet energy of $\mathcal{N}$ with that of a suitable harmonic extension of its boundary value on any given ball. 
(iii) In Section 5 we use the comparison above and a first variation argument to derive a suitable Poincaré-type inequality for $\mathcal{N}$.

(iv) In Section 6 we compute again the first variations of the Dirichlet energy of $\mathcal{N}$ and use the Poincaré inequality to bound efficiently several error terms.

(v) Using the latter bounds, in Section 7 we will prove an almost monotonicity property for the frequency function and the existence and boundedness of its limit, which is indeed the number $I_{0}$ of Theorem 2.8. The almost minimality of $\mathcal{N}$ will then allow us to conclude an exponential rate of decay to this limit.

(vi) From the decay of the previous step we will capture in Section 8 the asymptotic behaviour of $\mathcal{N}$ and show the existence of the map $g$ of Theorem 2.8.

The overall strategy follows the ideas and some of the computations in [3]. However several adjustments are needed to carry on the proof in the cases (b) and (c) of Definition 0.1. In particular in Section 7 we need to introduce a suitable modification of the usual frequency function to handle case (b).

2.3. Proof of the inductive step. In the next sections we will prove Theorem 2.8. We start observing that if case (a) of Theorem 1.8 does not hold, then we are necessarily in case (ii) of Theorem 2.8. Therefore we only need to prove that Theorem 2.8(ii) implies Theorem 1.8(b).

We divide the proof in different steps.

Step 1. For a reason which will become clear later, it is convenient to slightly modify the map $g$ to a multivalued map $n(z, w)=\sum_{i} \llbracket n_{i}(z, w) \rrbracket$ in such a way that $n_{i}(z, w)$ is orthogonal to $\mathcal{M}$ at $\boldsymbol{\Psi}(z, w)$. To achieve this it suffices to project $g_{i}(z, w)=\left(0, \bar{g}_{i}(z, w), 0\right)$ on the normal bundle. Observe that, by the estimates on $\left|A_{\mathcal{M}}\right|$ and $\boldsymbol{\Psi}$, we have

$$
\begin{aligned}
\left|g_{i}(z, w)-n_{i}(z, w)\right| & \leq C C_{i}|z|^{\gamma_{0}}\left|g_{i}(z, w)\right| \\
|D n|(z, w) & \leq|D g|(z, w)+C C_{i}|z|^{\gamma_{0}-1}|g|(z, w) .
\end{aligned}
$$

We introduce the function $H: \mathfrak{B}_{\bar{Q}} \rightarrow \mathcal{A}_{Q}\left(\mathbb{R}^{2+n}\right)$ given by

$$
H(z, w)=\sum_{i=1}^{Q} \llbracket H_{i}(z, w) \rrbracket:=\sum_{i=1}^{Q} \llbracket \mathbf{\Psi}(z, w)+n_{i}(z, w) \rrbracket .
$$

Note that, since $g$ is $I_{0}$-homogeneous and Dir-minimizer, by [4, Proposition 5.1] there is a constant $C$ such that

$$
\left|g_{i}(z, w)-g_{j}(z, w)\right| \geq 2 C|z|^{I_{0}} \quad \text { whenever } g_{i}(z, w) \neq g_{j}(z, w) .
$$

In fact [4, Proposition 5.1] is stated for maps with domain $\mathbf{C}=\mathbb{R}^{2}$. However, if we define the map $\mathbf{W}: \mathbb{C} \rightarrow \mathfrak{B}_{\bar{Q}, \infty}$ as $\mathbf{W}(z)=\left(z^{\bar{Q}}, z\right)$, by the conformality of $\mathbf{W}$ it is easy to check that $g \circ \mathbf{W}$ is Dir-minimizer and $I_{0} Q$ homogeneous.

By (2.20) and (2.22), provided $z$ is small enough we have

$$
\left|H_{i}(z, w)-H_{j}(z, w)\right| \geq C|z|^{I_{0}} \quad \text { whenever } H_{i}(z, w) \neq H_{j}(z, w) .
$$


Let $\bar{a} \in] 0, a_{0}\left[\right.$ be a constant to be fixed momentarily and $\zeta:=I_{0}+\bar{a} / 2>1$. Set

$$
\mathbf{V}_{H, \zeta}:=\left\{H_{i}(z, w)+p \in \mathbb{R}^{2+n}:|p|<|z|^{\zeta}, i=1, \ldots, Q\right\} .
$$

We claim that there exists $s>0$ such that $\operatorname{spt}(T) \cap \mathbf{B}_{s} \backslash\{0\} \subset \mathbf{V}_{H, \zeta}$.

In order to prove this claim, we distinguish two cases. First we consider any point $p \in \operatorname{spt}(T) \cap \operatorname{spt}\left(\mathbf{T}_{\mathcal{F}}\right) \backslash\{0\}$. In this case $p=\boldsymbol{\Psi}(z, w)+\mathcal{N}_{i}(z, w)$ for some $(z, w) \in$ $\mathfrak{B}_{\bar{Q}} \backslash\{0\}$ and for some $i=1, \ldots, Q$. Without loss of generality, by (2.16) we can assume $\left|\mathcal{N}_{i}(z, w)-g_{i}(z, w)\right| \leq C|z|^{I_{0}+\bar{a}}$, i.e.

$$
\begin{aligned}
\left|p-H_{i}(z, w)\right| & =\left|\mathcal{N}_{i}(z, w)-n_{i}(z, w)\right| \leq\left|\mathcal{N}_{i}(z, w)-g_{i}(z, w)\right|+\left|g_{i}(z, w)-n_{i}(z, w)\right| \\
& \leq C|z|^{I_{0}+\bar{a}}+C|z|^{\gamma_{0}+I_{0}}
\end{aligned}
$$

which in particular implies $\operatorname{spt}(T) \cap \operatorname{spt}\left(\mathbf{T}_{\mathcal{F}}\right) \cap \mathbf{B}_{s} \subset \mathbf{V}_{H, \zeta}$ if $s$ is sufficiently small and we impose $\frac{\bar{a}}{2}<\gamma_{0}$.

For the second case we consider a point $p \in \operatorname{spt}(T) \backslash \operatorname{spt}\left(\mathbf{T}_{\mathcal{F}}\right)$ and assume by contradiction that $p \notin \mathbf{V}_{H, \zeta}$. In particular, in view of (2.24) we have that

$$
B:=\mathbf{B}_{\frac{|z| \zeta}{2}}(p) \cap \operatorname{spt}\left(\mathbf{T}_{\mathcal{F}}\right)=\emptyset
$$

if $|z|$ is sufficiently small. By the monotonicity formula we know that $\|T\|(B) \geq C|z|^{2 \zeta}$; nevertheless since $B \subset \mathbf{P}^{-1}\left(B_{2|z|} \backslash B_{|z| / 2}\right),(2.25)$ implies $\|T\|(B) \leq\left\|T-\mathbf{T}_{\mathcal{F}}\right\|(B)$ and from (2.15) and (2.19) we conclude $\|T\|(B) \leq C|z|^{2 I_{0}+2 \kappa}$ with $\kappa=\min \left\{2 \eta_{0} I_{0}, \gamma_{0}\right\}$. This gives a contradiction if $\bar{a}<2 \kappa$.

Step 2. From the previous step we can infer that $g$ is a constant multiple of an irreducible function, namely there exists $Q^{\prime}>0$ such that $\operatorname{card}(g(z, w))=Q^{\prime}$ for every $(z, w) \neq(0,0)$ and there exists a continuous map $h: \mathfrak{B}_{\bar{Q} Q^{\prime}} \rightarrow \mathbb{R}^{2+n}$ such that

$$
g(z, w)=\frac{Q}{Q^{\prime}} \sum_{\tilde{z}=z, \tilde{w} Q^{\prime}=w} \llbracket h(\tilde{z}, \tilde{w}) \rrbracket .
$$

If this is not the case, by [4, Proposition 5.1] we can decompose $g$ in the superposition of irreducible functions, i.e. there exists a unique decomposition $g=\sum_{j=1}^{J} k_{j} g_{j}$ where $g_{j}: \mathfrak{B}_{Q} \rightarrow \mathcal{A}_{q_{j}}\left(\mathbb{R}^{n}\right)$ are Dir-minimizing $I_{0}$-homogeneous functions, for some choice of positive integers $J, k_{j}, q_{j}$ such that $\sum_{j=1}^{J} k_{j} q_{j}=Q$.

Denoting by $H^{j}$ the corresponding maps

$$
H^{j}(z, w):=\sum_{l=1}^{q_{j}} \llbracket \mathbf{\Psi}(z, w)+\left(n^{j}\right)_{l}(z, w) \rrbracket
$$

and by $\mathbf{V}_{H^{j}, \zeta}$ the corresponding horned neighborhoods

$$
\mathbf{V}_{H^{j}, \zeta}:=\left\{\left(H^{j}\right)_{l}(z, w)+p \in \mathbb{R}^{2+n}:|p|<|z|^{\zeta}, l=1, \ldots, q_{j}\right\},
$$

it follows from (2.23) that the closures of the $V_{\zeta, H_{i}}$ intersect only at the origin. Setting $T_{i}:=$ $T\left\llcorner V_{\zeta, H_{i}}\right.$, we infer that $T=\sum_{i} T_{i}$ with $\operatorname{spt}\left(T_{i}\right) \cap \operatorname{spt}\left(T_{j}\right)=\{0\}$, against the irreducibility of $T$. Note that, since $\boldsymbol{\eta} \circ g=0$ it also follows that $Q^{\prime}>1$. 
Having established (2.26), let us define $\boldsymbol{\Theta}: \mathfrak{B}_{\bar{Q} Q^{\prime}} \rightarrow \mathbb{R}^{2+n}$ as

$$
\Theta(\tilde{z}, \tilde{w}):=\mathbf{\Psi}\left(\tilde{z}, \tilde{w}^{Q^{\prime}}\right)+h^{n}(\tilde{z}, \tilde{w}) \quad \forall(\tilde{z}, \tilde{w}) \in \mathfrak{B}_{\bar{Q} Q^{\prime}}
$$

where $h^{n}(\tilde{z}, \tilde{w})$ is the projection of $h(\tilde{z}, \tilde{w})$ on the space normal to $\mathcal{M}$ at the point $\boldsymbol{\Psi}\left(\tilde{z}, \tilde{w}^{Q^{\prime}}\right)$. It follows that $\operatorname{Im}(H)=\operatorname{Im}(\boldsymbol{\Theta})$ is an admissible $\bar{Q} Q^{\prime}$-branching (the Hölder regularity for the graphical parametrization follows from the fact that $I_{0}>1$ ). Moreover, from the homogeneity of $g$ we easily infer that $\operatorname{Im}(\boldsymbol{\Theta})$ is $I_{0}$-separated (for a suitable constant $c_{s}$ ). Note that for $\zeta^{\prime}:=I_{0}+\bar{a} / 4$ and $s$ sufficiently small $\mathbf{V}_{H, \zeta} \cap \mathbf{B}_{s} \subset \mathbf{V}_{\boldsymbol{\Theta}, \zeta^{\prime}} \cap \mathbf{B}_{s}$.

Step 3. Finally we prove the condition (Dec) of Assumption 1.7. Let $(z, w) \in \mathfrak{B}_{\bar{Q}}$ with $0<|z|<\sqrt{2}$, let $V$ be the connected component of $\mathbf{V}_{\Theta, \zeta^{\prime}} \cap\{(x, y):|x-z|<d\}$ with $d:=|z| / 4$ containing $\Theta(z, w)$, and $p \in \operatorname{spt}(T) \cap V$ with coordinates $p=(z, y)$. Denote by $\pi$ the oriented two-vector for $\operatorname{Im}(\boldsymbol{\Theta})$ at $\boldsymbol{\Theta}(z, w)$, and consider $\rho \in\left[\frac{1}{2} d^{\left(I_{0}+1\right) / 2}, d\right]$.

Since $\mathbf{B}_{\rho}(p) \cap \operatorname{spt}(T) \subset \mathbf{P}^{-1}\left(\mathbf{\Psi}\left(B_{|z|+2 \rho} \backslash B_{|z|-2 \rho}\right)\right)$, we start estimating as follows

$$
\begin{aligned}
\int_{\mathbf{B}_{\rho}(p)}|\vec{T}-\vec{\pi}|^{2} d \| T\llcorner V \| & \leq \int_{\mathbf{B}_{\rho}(p) \cap V}\left|\overrightarrow{\mathbf{T}}_{\mathcal{F}}-\vec{\pi}\right|^{2} d\left\|\mathbf{T}_{\mathcal{F}}\right\|+\left\|T-\mathbf{T}_{\mathcal{F}}\right\|\left(\mathbf{p}^{-1}\left(B_{\left|x_{0}\right|+2 \rho}\right)\right) \\
& \stackrel{(2.15)}{\leq} \int_{\mathbf{B}_{\rho}(p) \cap V}\left|\overrightarrow{\mathbf{T}}_{\mathcal{F}}-\vec{\pi}\right|^{2} d\left\|\mathbf{T}_{\mathcal{F}}\right\|+C|z|^{2 I_{0}+2 \kappa}
\end{aligned}
$$

Next, note that for $|z|$ small enough $\mathbf{P}\left(\mathbf{B}_{\rho}(p) \cap \mathbf{V}_{\boldsymbol{\Theta}, \zeta^{\prime}}\right) \subset \mathbf{\Psi}\left(B_{2 \rho}(z, w)\right)$.

We can consider the set of indices $A \subset\{1, \ldots, Q\}$ such that $\mathcal{F}_{i}(z, w) \in V$ for $i \in A$ and estimate as follows

$$
\begin{aligned}
\int_{\mathbf{B}_{\rho}(p) \cap V}\left|\overrightarrow{\mathbf{T}}_{\mathcal{F}}-\vec{\pi}\right|^{2} d\left\|\mathbf{T}_{\mathcal{F}}\right\| \leq & C \sum_{i \in A} \int_{B_{2 \rho}(z, w)}\left|\overrightarrow{\mathbf{T}}_{\mathcal{F}_{i}(\zeta, \omega)}-\overrightarrow{\mathbf{T}}_{\boldsymbol{\Theta}(\zeta, \omega)}\right|^{2} d \zeta+C \rho^{2} \operatorname{Lip}\left(\left.D \Theta\right|_{B_{2 \rho}(z, w)}\right)^{2} \\
\leq & C \sum_{i \in A} \int_{B_{2 \rho}(z, w)}\left|\overrightarrow{\mathbf{T}}_{\mathcal{F}_{i}(\zeta, \omega)}-\overrightarrow{\mathbf{T}}_{\mathbf{\Psi}(\zeta, \omega)}\right|^{2} d \zeta \\
& +C \int_{B_{2 \rho}(z, w)}\left|\overrightarrow{\mathbf{T}}_{\boldsymbol{\Psi}(\zeta, \omega)}-\overrightarrow{\mathbf{T}}_{\boldsymbol{\Theta}(\zeta, \omega)}\right|^{2} d \zeta+C \rho^{4}|z|^{2 \theta-2},
\end{aligned}
$$

where $\theta:=\min \left\{\gamma_{0}, I_{0}-1\right\}$ and we used that $\left|D^{2} \boldsymbol{\Theta}\right|(z, w) \leq C|z|^{\theta-1}$.

We can finally use the computation of the excess in curvilinear coordinates in [5, Proposition 3.4] to get

$$
\begin{aligned}
\sum_{i} \int_{B_{2 \rho}(z, w)}\left|\overrightarrow{\mathbf{T}}_{\mathcal{F}_{i}(\zeta, \omega)}-\overrightarrow{\mathbf{T}}_{\boldsymbol{\Psi}(\zeta, \omega)}\right|^{2} & \leq C \int_{B_{2 \rho}(z, w)}\left(|D \mathcal{N}|^{2}+|\zeta|^{2 \gamma_{0}-2}|\mathcal{N}|^{2}\right) \\
& \stackrel{(2.19)}{\leq} C \int_{B_{|z|+2 \rho} \backslash B_{|z|-2 \rho}}|D \mathcal{N}|^{2}+C|z|^{2 I_{0}+2 \gamma_{0}} \\
& \stackrel{(2.17)}{\leq} C|z|^{2 I_{0}+a_{0}}+C|z|^{2 I_{0}-1} \rho
\end{aligned}
$$


and similarly

$$
\begin{aligned}
\int_{B_{2 \rho}(z, w)}\left|\overrightarrow{\mathbf{T}}_{\boldsymbol{\Theta}(\zeta, \omega)}-\overrightarrow{\mathbf{T}}_{\boldsymbol{\Psi}(\zeta, \omega)}\right|^{2} & \leq C \int_{B_{2 \rho}(z, w)}\left(|D n|^{2}+|\zeta|^{2 \gamma_{0}-2}|n|^{2}\right) \\
& \leq C \int_{B_{2 \rho}(z, w)}\left(|D g|^{2}+|\zeta|^{2 \gamma_{0}-2}|g|^{2}\right) \\
& \leq C|z|^{2 I_{0}-2} \rho^{2}+C|z|^{2 I_{0}+2 \gamma_{0}}
\end{aligned}
$$

(observe that, in order to apply [5, Proposition 3.4] we need that $n$ takes value into the normal bundle).

Collecting all the estimates together, we have that there exists a suitable constant $\varpi$ such that

$$
\int_{\mathbf{B}_{\rho}(p)}|\vec{T}-\vec{\pi}|^{2} d \| T\left\llcorner V \| \leq C|z|^{2 I_{0}+2 \varpi}+C \rho|z|^{2 I_{0}-1}+C \rho^{4}|z|^{2 \varpi-2} \leq|z|^{\gamma-2} \rho^{4},\right.
$$

where the last inequality is easily verified for $\gamma>0$ and $|z|$ small enough. This shows (Dec) in Assumption 1.7 and completes the proof.

\section{Dirichlet Almost minimizing PRoperty}

The normal approximation $\mathcal{N}$ inherits from $T$ an almost minimizing property for the Dirichlet energy, where the errors involved are in fact expressed in terms of some specific norms of $\mathcal{N}$ itself and of its competitors.

For techincal reasons we introduce the maps $F:=\sum_{i=1}^{Q} \llbracket p+N_{i}(p) \rrbracket$, where $N:=\mathcal{N} \circ \Psi^{-1}$. In order to state the almost minimizing property of $\mathcal{N}$ we introduce an appropriate notion of competitor.

Definition 3.1. A Lipschitz map $\mathcal{L}: B_{r} \rightarrow \mathcal{A}_{Q}\left(\mathbb{R}^{n+2}\right)$ is called a competitor for $\mathcal{N}$ in the ball $B_{r}$ if

(a) $\left.\mathcal{L}\right|_{\partial B_{r}}=\left.\mathcal{N}\right|_{\partial B_{r}}$

(b) $\operatorname{spt}(\mathcal{G}(z, w)) \subset \Sigma$ for all $(z, w) \in B_{r}$, where $\mathcal{G}(z, w):=\sum_{j=1}^{Q} \llbracket \Psi(z, w)+\mathcal{L}_{j}(z, w) \rrbracket$.

We are now ready to state the almost minimizing property for $\mathcal{N}$. We use the notation $\mathbf{p}_{T_{p} \Sigma}$ for the orthogonal projection on the tangent space to $\Sigma$ at $p$. We recall that, given our choice of coordinates, $\mathbf{p}_{T_{0} \Sigma}$ is the projection on $\mathbb{R}^{2+\bar{n}} \times\{0\}$. Since this projection will be used several times, we will denote it by $\mathbf{p}_{0}$. By the $C^{3, \varepsilon}$ regularity of $\Sigma$, there exists a $\operatorname{map} \Psi_{0} \in C^{3, \varepsilon}\left(\mathbb{R}^{2+\bar{n}}, \mathbb{R}^{l}\right)$ such that

$$
\Psi_{0}(0)=0, D \Psi_{0}(0)=0 \text { and } \operatorname{Gr}\left(\Psi_{0}\right)=\Sigma .
$$

Next, for each function $\mathcal{L}$ satisfying Condition (b) in Definition 3.1 we consider the map $\overline{\mathcal{L}}:=\mathbf{p}_{0} \circ \mathcal{L}$, which is a multivalued $\overline{\mathcal{L}}: \mathfrak{B} \rightarrow \mathcal{A}_{Q}\left(\mathbb{R}^{2+\bar{n}}\right)$. We observe that it is possible to determine $\mathcal{L}$ from $\overline{\mathcal{L}}$. In particular, fix coordinates $(\xi, \eta) \in \mathbb{R}^{2+\bar{n}} \times \mathbb{R}^{l}$ and let $\mathcal{L}=\sum \llbracket \mathcal{L}_{i} \rrbracket$, $\overline{\mathcal{L}}=\sum \llbracket \overline{\mathcal{L}}_{i} \rrbracket$, where $\overline{\mathcal{L}}_{i}=\mathbf{p}_{0} \circ \mathcal{L}_{i}$. Then the formula relating $\mathcal{L}_{i}$ and $\overline{\mathcal{L}}_{i}$ is

$$
\mathcal{L}_{i}(z, w)=\left(\overline{\mathcal{L}}_{i}(z, w), \Psi_{0}\left(\mathbf{p}_{0}(\mathbf{\Psi}(z, w))+\overline{\mathcal{L}}_{i}(z, w)\right)-\Psi_{0}\left(\mathbf{p}_{0}(\mathbf{\Psi}(z, w))\right) .\right.
$$


Proposition 3.2. There exists a constant $C_{3.2}>0$ such that the following holds. If $r \in(0,1)$ and $\mathcal{L}: B_{r} \rightarrow \mathcal{A}_{Q}\left(\mathbb{R}^{2+n}\right)$ is a Lipschitz competitor for $\mathcal{N}$ with $\|\mathcal{L}\|_{\infty} \leq r$ and $\operatorname{Lip}(\mathcal{L}) \leq C_{3.2}^{-1}$, then

$$
\int_{B_{r}}|D \mathcal{N}|^{2} \leq\left(1+C_{3.2} r\right) \int_{B_{r}}|D \overline{\mathcal{L}}|^{2}+C_{3.2} \operatorname{Err}_{1}\left(\mathcal{N}, B_{r}\right)+C_{3.2} \operatorname{Err}_{2}\left(\mathcal{L}, B_{r}\right)+C_{3.2} r^{2} \mathbf{D}^{\prime}(r),
$$

where $\overline{\mathcal{L}}:=\mathbf{p}_{0} \circ \mathcal{L}$ and the the error terms $\operatorname{Err}_{1}\left(\mathcal{N}, B_{r}\right), \operatorname{Err}_{2}\left(\mathcal{L}, B_{r}\right)$ are given by the following expressions:

$$
\operatorname{Err}_{1}\left(\mathcal{N}, B_{r}\right)=\Lambda^{\eta_{0}}(r) \mathbf{D}(r)+\mathbf{F}(r)+\mathbf{H}(r)+\boldsymbol{m}_{0}^{1 / 2} r^{1+\gamma_{0}} \int_{\partial B_{r}}|\boldsymbol{\eta} \circ \mathcal{N}|
$$

and

$$
\operatorname{Err}_{2}\left(\mathcal{L}, B_{r}\right)=\boldsymbol{m}_{0}^{1 / 2} \int_{B_{r}}|z|^{\gamma_{0}-1}|\boldsymbol{\eta} \circ \mathcal{L}|
$$

For the proof of Proposition 3.2 we consider separately the three cases:

(a) $T$ is mass minimizing;

(b) $T$ is semicalibrated;

(c) $T$ is the cross-section of a mass minimizing three-dimensional cone.

For notational convenience we set $L:=\mathcal{L} \circ \Psi^{-1}, G:=\mathcal{G} \circ \Psi^{-1}$.

Observe also that, by Lemma A.1 and A.2, it is enough to prove that

$\int_{B_{r}}|D \mathcal{N}|^{2} \leq\left(1+C_{3.2} r\right) \int_{B_{r}}|D \mathcal{L}|^{2}+C \operatorname{Err}_{1}\left(\mathcal{N}, B_{r}\right)+C \operatorname{Err}_{2}\left(\mathcal{L}, B_{r}\right)+\frac{C}{r} \int_{B_{r}}|\mathcal{L}|^{2}+C r^{2} \mathbf{D}^{\prime}(r)$.

Indeed Lemma A.2 implies that

$$
\begin{aligned}
\int_{B_{r}}|D \mathcal{L}|^{2} & \leq(1+C r) \int_{B_{r}}|D \overline{\mathcal{L}}|^{2}+C r \int_{\partial B_{r}}|\overline{\mathcal{L}}|^{2} \leq(1+C r) \int_{B_{r}}|D \overline{\mathcal{L}}|^{2}+C r \int_{\partial B_{r}}|\mathcal{L}|^{2} \\
& =(1+C r) \int_{B_{r}}|D \overline{\mathcal{L}}|^{2}+C r \int_{\partial B_{r}}|\mathcal{N}|^{2} \leq(1+C r) \int_{B_{r}}|D \overline{\mathcal{L}}|^{2}+C \operatorname{Err}_{1}\left(\mathcal{N}, B_{r}\right),
\end{aligned}
$$

whereas Lemma A.1 implies

$$
\frac{1}{r} \int_{B_{r}}|\mathcal{L}|^{2} \leq C r \int_{B_{r}}|D \mathcal{L}|^{2}+C \int_{\partial B_{r}}|\mathcal{L}|^{2} \leq C r \int_{B_{r}}|D \overline{\mathcal{L}}|^{2}+C \operatorname{Err}_{1}\left(\mathcal{N}, B_{r}\right) .
$$

3.1. Proof of Proposition 3.2 case (a): $T$ mass minimizing. We fix $\mathcal{L}, \overline{\mathcal{L}}, L, \mathcal{G}, \overline{\mathcal{G}}$ and $G$ as above. Let us set

$$
Z:=T-\mathbf{T}_{\left.\mathcal{F}\right|_{B_{r}}}+\mathbf{T}_{\mathcal{G}}
$$


Since $\left.\mathcal{F}\right|_{\partial B_{r}}=\left.\mathcal{G}\right|_{\partial B_{r}}$, from [5] it follows that $\partial\left(\mathbf{T}_{\mathcal{G}}-\mathbf{T}_{\mathcal{F}_{B_{r}}}\right)=0$. Moreover $\operatorname{spt}(Z) \subset \Sigma$ and therefore we must have $\mathbf{M}(T) \leq \mathbf{M}(Z)$. Taking into account (2.15), we conclude that

$$
\begin{aligned}
\mathbf{M}\left(\mathbf{T}_{\left.\mathcal{F}\right|_{B_{r}}}\right) & \leq \mathbf{M}\left(T\left\llcorner\mathbf{p}^{-1}\left(\boldsymbol{\Psi}\left(B_{r}\right)\right)\right)+\left\|T-\mathbf{T}_{\left.\mathcal{F}\right|_{B_{r}}}\right\|\left(\mathbf{p}^{-1}\left(\mathbf{\Psi}\left(B_{r}\right)\right)\right)\right. \\
& \leq \mathbf{M}\left(\mathbf{T}_{\mathcal{G}}\right)+2\left\|T-\mathbf{T}_{\left.\mathcal{F}\right|_{B_{r}}}\right\|\left(\mathbf{p}^{-1}\left(\mathbf{\Psi}\left(B_{r}\right)\right)\right) \\
& \leq \mathbf{M}\left(\mathbf{T}_{\mathcal{G}}\right)+C \operatorname{Err}_{1}\left(\mathcal{N}, B_{r}\right)
\end{aligned}
$$

Observe now that $\mathbf{T}_{\left.\mathcal{F}\right|_{B_{r}}}=\mathbf{T}_{\left.F\right|_{\Psi}\left(B_{r}\right)}$ and we can use the Taylor expansion in [5] to compute:

$$
\begin{aligned}
\mathbf{M}\left(\mathbf{T}_{\left.\mathcal{F}\right|_{B_{r}}}\right) \geq & Q \mathcal{H}^{2}\left(\boldsymbol{\Psi}\left(B_{r}\right)\right)+\frac{1}{2} \int_{\boldsymbol{\Psi}\left(B_{r}\right)}|D N|^{2}-Q \int_{\boldsymbol{\Psi}\left(B_{r}\right)}\left\langle\boldsymbol{\eta} \circ N, H_{\mathcal{M}}\right\rangle \\
& -C \int_{\boldsymbol{\Psi}\left(B_{r}\right)}\left(\left|A_{\mathcal{M}}\right|^{2}|N|^{2}+|D N|^{4}\right)
\end{aligned}
$$

where $H_{\mathcal{M}}$ denotes the mean curvature vector of $\mathcal{M}$. Note that in order to apply the Taylor expansion in [5] we need the manifold $\mathcal{M}$ to be $C^{2}$, with an apriori bound on the $C^{2}$ norm. However, if we take $T_{F}\left\llcorner\mathbf{B}_{r} \backslash \mathbf{B}_{r / 2}\right.$ and rescale by a factor $1 / r$, the corresponding rescaled current, map and manifold fall under the assumptions of the Taylor expansion in [5]. We can then scale back to find the corresponding inequalities for $T\left\llcorner\mathbf{B}_{r} \backslash \mathbf{B}_{r / 2}\right.$ and sum over dyadic annuli to conclude (3.8).

Using the conformality of $\boldsymbol{\Psi}$ we conclude

$$
\int_{\Psi\left(B_{r}\right)}|D N|^{2}=\int_{B_{r}}|D \mathcal{N}|^{2}
$$

As for the other terms, we recall

$$
\begin{aligned}
& \int_{\boldsymbol{\Psi}\left(B_{r}\right)}\left|\left\langle\boldsymbol{\eta} \circ N, H_{\mathcal{M}}\right\rangle\right| \leq C \boldsymbol{m}_{0}^{1 / 2} \int_{B_{r}}|\boldsymbol{\eta} \circ \mathcal{N}| \stackrel{(2.14)}{\leq} C \operatorname{Err}_{1}\left(\mathcal{N}, B_{r}\right), \\
& \int_{\boldsymbol{\Psi}\left(B_{r}\right)}|D N|^{4} \leq C \operatorname{Lip}\left(\left.\mathcal{N}\right|_{B_{r}}\right)^{2} \int_{B_{r}}|D \mathcal{N}|^{2} \stackrel{(2.13)}{\leq} C \operatorname{Err}_{1}\left(\mathcal{N}, B_{r}\right), \\
& \int_{\boldsymbol{\Psi}\left(B_{r}\right)}\left|A_{\mathcal{M}}\right|^{2}|N|^{2} \leq C \boldsymbol{m}_{0} \int_{B_{r}}|z|^{2 \gamma_{0}-2}|\mathcal{N}|^{2}=C \boldsymbol{m}_{0} \int_{0}^{r} \frac{\mathbf{H}(s)}{s^{2-2 \gamma_{0}}} d s \leq C \operatorname{Err}_{1}\left(\mathcal{N}, B_{r}\right) .
\end{aligned}
$$

Combining the latter estimates with (3.6) and (3.7) we achieve

$$
\frac{1}{2} \int_{B_{r}}|D \mathcal{N}|^{2} \leq C \operatorname{Err}_{1}\left(\mathcal{N}, B_{r}\right)+\mathbf{M}\left(\mathbf{T}_{G}\right)-Q \mathcal{H}^{2}\left(\mathbf{\Psi}\left(B_{r}(x)\right)\right.
$$


Next, fix an orthonormal frame $\xi_{1}, \xi_{2}$ on $B_{r}$ and, using the area formula from [5], compute

$$
\begin{aligned}
\mathbf{M}\left(\mathbf{T}_{G}\right)= & \int_{\boldsymbol{\Psi}\left(B_{r}\right)} \sum_{i}\left|\left(\xi_{1}+D L_{i} \cdot \xi_{1}\right) \wedge\left(\xi_{2}+D L_{i} \cdot \xi_{2}\right)\right| \\
\leq & \left.\left.\frac{1}{2} \int_{\boldsymbol{\Psi}\left(B_{r}\right)} \sum_{i}\left(\mid \xi_{1}+D L_{i} \cdot \xi_{1}\right)\right|^{2}+\left|\xi_{2}+D L_{i} \cdot \xi_{2}\right|^{2}\right) \\
= & Q \mathcal{H}^{2}\left(\boldsymbol{\Psi}\left(B_{r}\right)\right)+\frac{1}{2} \int_{\boldsymbol{\Psi}\left(B_{r}\right)}|D L|^{2} \\
& \quad+Q \int_{\boldsymbol{\Psi}\left(B_{r}\right)}\left(\left\langle D(\boldsymbol{\eta} \circ L) \cdot \xi_{1}, \xi_{1}\right\rangle+\left\langle D(\boldsymbol{\eta} \circ L) \cdot \xi_{2}, \xi_{2}\right\rangle\right) .
\end{aligned}
$$

By conformality the second summand in the last inequality equals $\frac{1}{2} \int_{B_{r}}|D \mathcal{L}|^{2}$. We integrate by parts the third summand. Recall that $\boldsymbol{\eta} \circ L=\boldsymbol{\eta} \circ N$ on $\boldsymbol{\Psi}\left(\partial B_{r}\right)=\partial\left(\boldsymbol{\Psi}\left(B_{r}\right)\right)$ : since $\boldsymbol{\eta} \circ N$ is orthogonal to $\xi_{i}$ the boundary term vanishes. Moreover, since the origin is a singularity, we must in fact integrate by parts in $B_{r} \backslash B_{\varepsilon}$ and then let $\varepsilon \rightarrow 0$. A specific choice of $\xi_{i}$ is $\xi_{i}=\lambda^{-1 / 2} D \Psi \cdot e_{i}$, where $e_{1}, e_{2}$ is the parallel frame on $\mathfrak{B}_{Q}$ naturally induced by the standard flat coordinates. It then turns out that

$$
\left|D_{\xi_{1}} \xi_{1}+D_{\xi_{2}} \xi_{2}\right|(\boldsymbol{\Psi}(z, w)) \leq C \boldsymbol{m}_{0}^{1 / 2}|z|^{\gamma_{0}-1} .
$$

In particular $\left|D_{\xi_{1}} \xi_{1}+D_{\xi_{2}} \xi_{2}\right|$ is integrable on $B_{r}$ and we can therefore conclude

$$
\begin{aligned}
\mathbf{M}\left(\mathbf{T}_{G}\right)-Q \mathcal{H}^{2}\left(\boldsymbol{\Psi}\left(B_{r}\right)\right) & \leq \frac{1}{2} \int_{\boldsymbol{\Psi}\left(B_{r}\right)}|D L|^{2}+Q \int_{\boldsymbol{\Psi}\left(B_{r}\right)}\left\langle\boldsymbol{\eta} \circ L, D_{\xi_{1}} \xi_{1}+D_{\xi_{2}} \xi_{2}\right\rangle \\
& \leq \frac{1}{2} \int_{B_{r}}|D \mathcal{L}|^{2}+C \operatorname{Err}_{2}\left(\mathcal{L}, B_{r}\right) .
\end{aligned}
$$

Combining (3.12) and (3.13) we conclude (3.5).

3.2. Proof of Proposition 3.2 case (b): $T$ semicalibrated. We proceed as in the previous step and define the current $Z$ as in (3.6). If $S$ is any current such that

$$
\partial S=T-Z=\mathbf{T}_{\mathcal{F}_{B_{r}}}-\mathbf{T}_{\mathcal{G}}=\mathbf{T}_{\left.F\right|_{\Psi(B r)}}-\mathbf{T}_{G}
$$

then the semicalibrated condition gives

$$
\mathbf{M}(T) \leq \mathbf{M}(Z)+S(d \omega)
$$

where $\omega$ is the calibrating form. In particular, in order to conclude the proof it suffices to find an $S$ such that

$$
|S(d \omega)| \leq C \operatorname{Err}_{1}\left(\mathcal{N}, B_{r}\right)+C \operatorname{Err}_{2}\left(\mathcal{L}, B_{r}\right)+\frac{C}{r} \int_{B_{r}}|\mathcal{L}|^{2}:
$$

combining the latter inequality with the estimates of the previous subsection we reach the desired inequality. 
We first define $H_{i}:[0,1] \times \boldsymbol{\Psi}\left(B_{r}\right) \rightarrow \mathcal{A}_{Q}\left(\mathbb{R}^{2+n}\right)$ for $i=1,2$ by

$$
\begin{gathered}
{[0,1] \times \Psi\left(B_{r}\right) \ni(t, p) \mapsto H_{1}(t, p):=\sum_{i=1}^{Q} \llbracket p+t N_{i}(p) \rrbracket \in \mathcal{A}_{Q}\left(\mathbb{R}^{2+n}\right)} \\
{[0,1] \times \boldsymbol{\Psi}\left(B_{r}\right) \ni(t, p) \mapsto H_{2}(t, p):=\sum_{i=1}^{Q} \llbracket p+(1-t) L_{i}(p) \rrbracket \in \mathcal{A}_{Q}\left(\mathbb{R}^{2+n}\right) .}
\end{gathered}
$$

We choose $S:=S_{1}+S_{2}$, where $S_{i}:=\mathbf{T}_{H_{i}}$ for $i=1,2$. Thanks to the homotopy formula in [5], we get

$$
\begin{aligned}
& \partial S_{1}=\mathbf{T}_{\left.F\right|_{\Psi\left(B_{r}\right)}}-Q \llbracket \mathcal{M} \rrbracket-\mathbf{T}_{\left.H_{1}\right|_{[0,1] \times \Psi\left(\partial B_{r}\right)}}, \\
& \partial S_{2}=Q \llbracket \mathcal{M} \rrbracket-\mathbf{T}_{\left.G\right|_{\Psi\left(B_{r}\right)}}+\mathbf{T}_{\left.H_{2}\right|_{[0,1] \times \Psi\left(\partial B_{r}\right)}} .
\end{aligned}
$$

On the other hand since $N=L$ on $\Psi\left(\partial B_{r}\right)$, we conclude $\partial S=\partial\left(S_{1}+S_{2}\right)=T-Z$.

We next estimate $\left|S_{1}(d \omega)\right|$ and $\left|S_{2}(d \omega)\right|$. Since the estimates are analogous, we give the details only for the first. We start from the formula

$$
S_{1}(d \omega)=\int_{\Psi\left(B_{r}\right)} \int_{0}^{1} \sum_{i=1}^{Q}\left\langle\vec{\zeta}_{i}(t, p), d \omega\left(\left(H_{1}\right)_{i}(t, p)\right)\right\rangle d \mathcal{H}^{2}(p) d t
$$

with

$$
\begin{aligned}
\vec{\zeta}_{i}(t, p) & =\left(\xi_{1}+t \nabla_{\xi_{1}} N_{i}(p)\right) \wedge\left(\xi_{2}+t \nabla_{\xi_{2}} N_{i}(p)\right) \wedge N_{i}(p) \\
& =: \xi_{1} \wedge \xi_{2} \wedge N_{i}(p)+\vec{E}_{i}(t, p)
\end{aligned}
$$

and

$$
\left|\vec{E}_{i}(t, p)\right| \leq C\left(|D N|(p)+|D N|^{2}(p)\right)|N|(p) .
$$

Next we note that

$$
d \omega\left(\left(H_{1}\right)_{i}(t, p)\right)=d \omega(p)+I(t, p),
$$

where $I(t, p)$ can be estimated by

$$
|I(t, p)|=\left|d \omega\left(\left(H_{1}\right)_{i}(t, p)\right)-d \omega(p)\right| \leq C\left\|D^{2} \omega\right\|_{L^{\infty}}|N|(p) .
$$

Therefore, we have

$$
\begin{aligned}
& \left|\sum_{i=1}^{Q}\left\langle\vec{\zeta}_{i}(t, p), d \omega\left(\left(H_{1}\right)_{i}(t, p)\right)\right\rangle\right| \leq \sum_{i=1}^{Q}\left\langle\xi_{1} \wedge \xi_{2} \wedge N_{i}(p), d \omega(p)\right\rangle+\|d \omega\|_{L^{\infty}} \sum_{i=1}^{Q}\left|\vec{E}_{i}(t, p)\right| \\
& \quad+C \sum_{i=1}^{Q}\left(\left(\left|N_{i}\right|+\left|\vec{E}_{i}\right|\right)|I|\right)(t, p) \\
& \quad \leq C \boldsymbol{m}_{0}^{1 / 2}|\boldsymbol{\eta} \circ N|+C|N|^{2}(p)+C|D N|(p)|N|(p)+C r|D N|^{2}(p),
\end{aligned}
$$


where we have only used the bound $|N|(p) \leq C r$ on $\Psi\left(B_{r}\right)$. Arguing similarly for $S_{2}$ (observe that we have the bound $|L|(p) \leq C r$ ) and estimating $|N||D N|+|L||D L| \leq$ $r^{-1}\left(|N|^{2}+|L|^{2}\right)+C r\left(|D N|^{2}+|D L|^{2}\right)$, we achieve

$$
\begin{aligned}
\left|S_{1}(d \omega)\right|+\left|S_{2}(d \omega)\right| \leq & C \boldsymbol{m}_{0}^{1 / 2} \int_{\boldsymbol{\Psi}\left(B_{r}\right)}(|\boldsymbol{\eta} \circ N|+|\boldsymbol{\eta} \circ L|)+C r^{-1} \int_{\boldsymbol{\Psi}\left(B_{r}\right)}\left(|N|^{2}+|L|^{2}\right) \\
& +C r \int_{\boldsymbol{\Psi}\left(B_{r}\right)}\left(|D N|^{2}+|D L|^{2}\right),
\end{aligned}
$$

and we conclude as above by a change of variable and Theorem 2.6.

3.3. Proof of Proposition 3.2 in case (c): $T$ is the cross-section of a three dimensional area minimizing cone. Recall that in this case $\operatorname{spt}(T) \subset \partial \mathbf{B}_{R}\left(p_{0}\right)$, where $p_{0}=(0, \ldots, 0, R)=R e_{n+2}$ and $R^{-1} \leq \boldsymbol{m}_{0}^{1 / 2}$. For the computations of this subsection it is indeed convenient to change coordinates so that $p_{0}$ is in fact the origin, whereas $\Psi(0,0)$ is the point $(0, \ldots, 0,-R)$. In these new coordinates we then have $\mathcal{M}, \operatorname{spt}(T), \operatorname{Im}(\mathcal{F}) \subset$ $\partial \mathbf{B}_{R}(0)$. These coordinates will however be used only in here, whereas in the next sections we will return to the usual ones.

We introduce the following notation: $\mathcal{C}(r)$ is the cone over $\boldsymbol{\Psi}\left(B_{r}\right)$ with vertex 0, i.e.

$$
\mathcal{C}(r):=\left\{\rho p \in \mathbb{R}^{n+2}: \rho \in[0,1], p \in \mathbf{\Psi}\left(B_{r}\right)\right\}
$$

with the orientation compatible with that of $0 \nVdash \llbracket \mathcal{M} \rrbracket$. We extend $F$ to $\tilde{F}: \mathcal{C}(r) \rightarrow$ $\mathcal{A}_{Q}\left(\mathbb{R}^{n+2}\right)$ by setting $\tilde{F}(\rho p):=\rho F(p)$ for every $p \in \mathbf{\Psi}\left(B_{r}\right)$.

In order to estimate the Dirichlet energy of $N$ in terms of that of $L$, we construct a suitable function $K: \mathcal{C}(r) \rightarrow \mathcal{A}_{Q}\left(\mathbb{R}^{n+2}\right.$ ) (depending on $L$ and $N$ ) such that $\left.K\right|_{\partial \mathcal{C}(r)}=$ $\left.\tilde{F}\right|_{\partial \mathcal{C}(r)}$ : we can then test the minimizing property of $0 ※ T$ comparing its mass with that of the current

$$
Z:=0 ※ T-\mathbf{T}_{\tilde{F}}+\mathbf{T}_{K}=0 ※\left(T-\mathbf{T}_{\left.F\right|_{\left(B_{r}\right)}}\right)+\mathbf{T}_{K}
$$

which is easily recognized to satisfy $\partial Z=\partial(0 \times T)$. In particular, using the minimality of $0 ※ T$, we conclude

$$
R^{-1} \mathbf{M}\left(0 \nsim \mathbf{T}_{\left.F\right|_{\Psi\left(B_{r}\right)}}\right) \leq R^{-1} \mathbf{M}\left(\mathbf{T}_{K}\right)+C \operatorname{Err}_{1}\left(\mathcal{N}, B_{r}\right)
$$

We consider the space of parameters $[0,1] \times B_{r}$ and recall that the points in $\mathfrak{B}_{Q}$ are identified by two complex coordinates $(z, w) \in \mathbb{C} \times \mathbb{C}$. For the definition of $K$ we need to introduce the following sets

$$
\begin{aligned}
& A_{1}:=\left\{(\rho, z, w) \in[0,1] \times B_{r}: 1-r \leq \rho \leq 1,|z| \leq \frac{\rho+2 r-1}{2}\right\}, \\
& A_{2}:=\left\{(\rho, z, w) \in[0,1] \times B_{r}: 1-2 r \leq \rho \leq 1-r,|z| \leq \frac{1-\rho}{2}\right\}, \\
& B:=[1-2 r, 1] \times B_{r} \backslash\left(A_{1} \cup A_{2}\right),
\end{aligned}
$$


We then define the function $\mathcal{H}:[0,1] \times B_{r} \rightarrow \mathcal{A}_{Q}\left(\mathbb{R}^{n+2}\right)$ given by

$$
\mathcal{H}(\rho, z, w):= \begin{cases}\rho \mathcal{L}(z, w) & \text { if } \rho \leq 1-2 r, \\ \rho l_{1}(\rho) \mathcal{N}\left(\frac{2 r z}{\rho+2 r-1}, \frac{(2 r)^{1 / Q}}{(\rho+2 r-1)^{1 / Q}} w\right) & \text { if }(\rho, z, w) \in A_{1}, \\ -\rho l_{1}(\rho) \mathcal{L}\left(\frac{2 r z}{1-\rho}, \frac{(2 r)^{1 / Q}}{(1-\rho)^{1 / Q}} w\right) & \text { if }(\rho, z, w) \in A_{2}, \\ \rho l_{2}(|z|) \mathcal{N}\left(\frac{r z}{|z|}, \frac{r^{1 / Q}}{|z|^{1 / Q}} w\right) & \text { if }(\rho, z, w) \in B,\end{cases}
$$

where $l_{1}, l_{2}: \mathbb{R} \rightarrow \mathbb{R}$ are the affine functions

$$
l_{1}(t):=\frac{t+r-1}{r} \text { and } \quad l_{2}(t):=\frac{2 t-r}{r} .
$$

The following are simple properties of $\mathcal{H}$ which can be easily verified:

(1) $\mathcal{H}(1, z, w)=\mathcal{N}(z, w)$ for every $(z, w) \in B_{r}$, as $(1, z, w) \in A_{1}$ and $l_{1}(1)=1$;

(2) $\mathcal{H}(\rho, z, w)=\rho \mathcal{N}(z, w)$ for every $\rho \in[0,1]$ and for every $z$ with $|z|=r$, as $\left.\mathcal{L}\right|_{\partial B_{r}}=$ $\left.\mathcal{N}\right|_{\partial B_{r}}$ and $l_{2}(r)=1$

(3) $\mathcal{H}$ is well-defined and continuous, as $\mathcal{H} \equiv 0$ in $A_{1} \cap A_{2}$ from $l_{1}(1-r)=0$,

$$
\mathcal{H}(\rho, z, w)=\rho \frac{\rho+r-1}{r} \mathcal{N}\left(\frac{r z}{|z|}, \frac{r^{1 / Q}}{|z|^{1 / Q}} z\right) \quad \text { in } A_{1} \cap \partial B
$$

and

$$
\mathcal{H}(\rho, z, w)=\rho \frac{\rho+r-1}{r} \mathcal{N}\left(\frac{r z}{|z|}, \frac{r^{1 / Q}}{|z|^{1 / Q}} w\right) \quad \text { in } A_{2} \cap \partial B .
$$

The competitor map $K: \mathcal{C}(r) \rightarrow \mathcal{A}_{Q}\left(\mathbb{R}^{n+2}\right)$ is now given by

$$
K(\rho p):=\sum_{i=1}^{Q} \llbracket \rho p+H_{i}(\rho p) \rrbracket \quad \text { with } H(\rho p):=\mathcal{H}\left(\rho, \Psi^{-1}(p)\right) .
$$

Note that by (1) and (2) above it follows that $\left.K\right|_{\partial \mathcal{C}(r)}=\left.\tilde{F}\right|_{\partial \mathcal{C}(r)}$.

We start now estimating the masses of the various currents introduced above. Since $\operatorname{spt}\left(\mathbf{T}_{F}\right) \subset \partial \mathbf{B}_{R}(0)$, it follows that $\mathbf{M}\left(0 \mathbb{x} \mathbf{T}_{F}\right)=R \mathbf{M}\left(\mathbf{T}_{F}\right) / 3$ and, by the expansion of the mass of $\mathbf{T}_{F}$, we have that

$$
\mathbf{M}\left(\mathbf{T}_{\left.F\right|_{\Psi\left(B_{r}\right)}}\right) \geq Q \mathcal{H}^{2}\left(\mathbf{\Psi}\left(B_{r}\right)\right)+\frac{1}{2} \int_{B_{r}}|D \mathcal{N}|^{2}-C \operatorname{Err}_{1}\left(\mathcal{N}, B_{r}\right)
$$

Combining the latter estimate with (3.18) we conclude

$$
\int_{B_{r}}|D \mathcal{N}|^{2} \leq 6 R^{-1} \mathbf{M}\left(\mathbf{T}_{K}\right)-2 Q \mathcal{H}^{2}\left(\mathbf{\Psi}\left(B_{r}\right)\right)+C \operatorname{Err}_{1}\left(\mathcal{N}, B_{r}\right) .
$$

For what concerns the mass of $\mathbf{T}_{K}$, recalling that $p+\operatorname{spt}(L(p)) \in \partial \mathbf{B}_{R}(0)$ for every $p \in \boldsymbol{\Psi}\left(B_{r}\right)$, we deduce that

$$
\mathbf{M}\left(\mathbf{T}_{K}\left\llcorner\mathbf{B}_{R(1-2 r}\right)\right)=\mathbf{M}\left(0 ※ \mathbf{T}_{G}\left\llcorner\mathbf{B}_{R(1-2 r)}\right)=R \frac{(1-2 r)^{3} \mathbf{M}\left(\mathbf{T}_{G}\right)}{3}\right.
$$


and

$$
\mathbf{M}\left(\mathbf{T}_{G}\right) \leq Q \mathcal{H}^{2}\left(\Psi\left(B_{r}\right)\right)+\frac{1}{2} \int_{B_{r}}|D \mathcal{L}|^{2}+\operatorname{Err}_{2}\left(\mathcal{L}, B_{r}\right) .
$$

In particular we conclude

$$
6 R^{-1} \mathbf{M}\left(\mathbf{T}_{K}\left\llcorner\mathbf{B}_{R(1-2 r)}\right) \leq 2 Q(1-2 r)^{3} \mathcal{H}^{2}\left(\mathbf{\Psi}\left(B_{r}\right)\right)+\int_{B_{r}}|D \mathcal{L}|^{2}+\operatorname{Err}_{2}\left(\mathcal{L}, B_{r}\right) .\right.
$$

Next we pass to estimating $\mathbf{M}\left(\mathbf{T}_{K}\left\llcorner\mathbf{B}_{R} \backslash \mathbf{B}_{R(1-2 r)}\right)\right.$. In order to carry on our estimates we use the area formula for multifunctions, cf. [5]. In particular we fix an orthonormal frame $\xi_{1}, \xi_{2}$ for $\mathcal{M}$ as in the proof of case (a) and we let $\xi_{3}=R^{-1} \partial_{t}$ be normal to them in $T \mathcal{C}(r)$, i.e. pointing in the radial direction of the cone. We then have

$$
\mathbf{M}(\mathbf{T}_{K} \mathrm{~L}\left(\mathbf{B}_{R} \backslash \mathbf{B}_{R(1-2 r)}\right)=\int_{\mathcal{C}(r)} \sum_{i} \underbrace{\left|\left(\xi_{1}+D H_{i} \cdot \xi_{1}\right) \wedge\left(\xi_{2}+D H_{i} \cdot \xi_{2}\right) \wedge\left(\xi_{3}+D H_{i} \cdot \xi_{3}\right)\right|}_{(A)} .
$$

Using the Taylor expansion for $(A)$, cf. [5], we can bound

$$
\begin{aligned}
R^{-1} \mathbf{M} & \left(\mathbf{T}_{K}\left\llcorner\left(\mathbf{B}_{R} \backslash \mathbf{B}_{R(1-2 r)}\right)\right) \leq Q R^{-1} \mathcal{H}^{3}\left(\mathcal{C}(r) \cap \mathbf{B}_{1} \backslash \mathbf{B}_{1-2 r}\right)\right. \\
& +Q R^{-1} \int_{1-2 r}^{1} \int_{\boldsymbol{\Psi}\left(B_{r}\right)} \frac{d}{d t}[(\boldsymbol{\eta} \circ H)(t p)] t^{2} d t \\
& +Q R^{-1} \int_{1-2 r}^{1} \int_{\boldsymbol{\Psi}\left(B_{r}\right)} \sum_{i=1}^{2}\left\langle\nabla_{\xi_{i}}(\boldsymbol{\eta} \circ H), \xi_{i}\right\rangle t^{2} d t+C R^{-1} \int_{1-2 r}^{1} \int_{\boldsymbol{\Psi}\left(B_{r}\right)}|D H|^{2} t^{2} d t .
\end{aligned}
$$

The linear terms can be integrated by parts: since $\nabla_{p}(\boldsymbol{\eta} \circ H)(t p)=\frac{d}{d t}(\boldsymbol{\eta} \circ H)(t p)$, we have

$$
\begin{gathered}
\int_{1-2 r}^{1} \int_{\boldsymbol{\Psi}\left(B_{r}\right)} \frac{d}{d t}[(\boldsymbol{\eta} \circ H)(t p)] t^{2} d t= \\
\int_{\boldsymbol{\Psi}\left(B_{r}\right)}\left\langle(\boldsymbol{\eta} \circ H)(p)-(1-2 r)^{2}(\boldsymbol{\eta} \circ H)((1-2 r) p), p\right\rangle \\
-2 \int_{1-2 r}^{1} \int_{\boldsymbol{\Psi}\left(B_{r}\right)}\langle(\boldsymbol{\eta} \circ H)(t p), p\rangle t d t \\
\int_{1-2 r}^{1} \int_{\boldsymbol{\Psi}\left(B_{r}\right)} \sum_{i=1}^{2}\left\langle\nabla_{\xi_{i}}(\boldsymbol{\eta} \circ H), \xi_{i}\right\rangle t^{2} d t=-\int_{1-2 r}^{1} \int_{\boldsymbol{\Psi}\left(B_{r}\right)}\left\langle(\boldsymbol{\eta} \circ H), H_{\mathcal{M}}\right\rangle t^{2} d t
\end{gathered}
$$

Therefore, by a simple change of coordinates we can estimate

$$
\begin{aligned}
R^{-1} \mathbf{M}\left(\mathbf{T}_{K}\left\llcorner\left(\mathbf{B}_{R} \backslash \mathbf{B}_{R(1-r)}\right)\right) \leq \frac{Q\left(1-(1-2 r)^{3}\right)}{3} \mathcal{H}^{2}\left(\boldsymbol{\Psi}\left(B_{r}\right)\right)\right. \\
\quad+C \boldsymbol{m}_{0}^{1 / 2} \int_{B_{r}}(|\boldsymbol{\eta} \circ \mathcal{N}|+|\boldsymbol{\eta} \circ \mathcal{L}|)+C \boldsymbol{m}_{0}^{1 / 2} \int_{1-2 r}^{1} \int_{B_{r}}|D \mathcal{H}|^{2}(t, z, w) d z d t \\
\quad+C \boldsymbol{m}_{0}^{1 / 2} \int_{1-2 r}^{1} \int_{B_{r}}|z|^{\gamma_{0}-1}|\boldsymbol{\eta} \circ \mathcal{H}|(t, z, w) d z d t .
\end{aligned}
$$


In order to bound the various integrands of (3.30), we start with the following general remark. Assume that $\chi:[1-2 r, 1] \times B_{r} \rightarrow[0,+\infty)$ has the structure

$$
\chi(\rho, x, y)= \begin{cases}\chi_{1}\left(\frac{2 r z}{\rho+2 r-1}, \frac{(2 r)^{1 / Q}}{(\rho+2 r-1)^{1 / Q}} w\right) & \text { if }(\rho, z, w) \in A_{1}, \\ \chi_{2}\left(\frac{2 r z}{1-\rho}, \frac{(2 r)^{1 / Q}}{(1-\rho)^{1 / Q}} w\right) & \text { if }(\rho, z, w) \in A_{2}, \\ \chi_{3}\left(\frac{r z}{|z|}, \frac{r^{1 / Q}}{|z|^{1 / Q}} w\right) & \text { if }(\rho, z, w) \in B,\end{cases}
$$

for some $\chi_{1}, \chi_{2}, \chi_{3}: B_{r} \rightarrow[0,+\infty)$. Then one can compute the integral of $\chi$ in the following way:

$$
\int_{1-2 r}^{1} \int_{B_{r}} \chi(t, z, w) d z d t=\int_{A_{1}} \chi(t, z, w) d z d t+\int_{A_{2}} \chi(t, z, w) d z d t+\int_{B} \chi(t, z, w) d z d t
$$

and one can easily compute that

$$
\begin{aligned}
& \int_{A_{1}} \chi(t, z, w) d z d t=\int_{1-r}^{1} \int_{B_{\frac{t+2 r-1}{2}}} \chi_{1}(t, z, w) d z d t \\
= & \int_{1-r}^{1} \int_{B_{\frac{t+2 r-1}{2}}} \chi_{1}\left(\frac{2 r z}{t+2 r-1}, \frac{(2 r)^{1 / Q}}{(t+2 r-1)^{1 / Q}} w\right) d z d t \\
= & \int_{1-r}^{1}\left(\frac{t+2 r-1}{2 r}\right)^{2} \int_{B_{r}} \chi_{1}(z, w) d z d t \leq r \int_{B_{r}} \chi_{1}(z, w) d z d t .
\end{aligned}
$$

Similarly

$$
\int_{A_{2}} \chi(t, z, w) d z d t \leq r \int_{B_{r}} \chi_{2}(z, w) d t
$$

and

$$
\begin{aligned}
\int_{B} \chi(t, z, w) d z d t= & \int_{1-r}^{1} d t \int_{\frac{t+2 r-1}{2}}^{r} \frac{s}{r} d s \int_{\partial B_{r}} \chi_{3}(z, w) d z \\
& +\int_{1-2 r}^{1-r} \int_{\frac{1-t}{2}} r \frac{s}{r} d s \int_{\partial B_{r}} \chi_{3}(z, w) d z \leq r^{2} \int_{\partial B_{r}} \chi_{3}(z, w) d z .
\end{aligned}
$$

By direct computations one verifies that the integrands in (3.30) are all bounded from above by functions $\chi$ with the structure (3.33): in particular,

(i) $|z|^{\gamma_{0}-1}|\boldsymbol{\eta} \circ \mathcal{H}|(t, z, w) \leq \chi(t, z, w)$ if we choose

$$
\chi_{1}(z, w)=\chi_{3}(z, w)=|z|^{\gamma_{0}-1}|\boldsymbol{\eta} \circ \mathcal{N}|(z, w) \quad \text { and } \quad \chi_{2}(z, w)=|z|^{\gamma_{0}-1}|\boldsymbol{\eta} \circ \mathcal{L}|(x, y) \text {; }
$$

(ii) $|D \mathcal{H}|^{2}(t, z, w) \leq \chi(t, z, w)$ if we choose

$$
\begin{gathered}
\chi_{1}(z, w)=\chi_{3}(z, w)=\frac{C}{r^{2}}|\mathcal{N}|^{2}(z, w)+C|D \mathcal{N}|^{2}(z, w) \\
\chi_{2}(z, w)=\frac{C}{r^{2}}|\mathcal{L}|^{2}(z, w)+C|D L|^{2}(z, w) .
\end{gathered}
$$


for some dimensional constant $C>0$.

It then turns out from (3.34), (3.35), (3.36) and (i), (ii), (iii) that

$$
\begin{aligned}
6 R^{-1} \mathbf{M}\left(\mathbf{T}_{K}\left\llcorner\left(\mathbf{B}_{R} \backslash \mathbf{B}_{R(1-r)}\right)\right) \leq Q\right. & \left(1-(1-2 r)^{3}\right) \mathcal{H}^{2}\left(\mathbf{\Psi}\left(B_{r}\right)\right) \\
& +C \operatorname{Err}_{1}\left(\mathcal{N}, B_{r}\right)+C \operatorname{Err}_{2}\left(\mathcal{L}, B_{r}\right) .
\end{aligned}
$$

Summing (3.37) and (3.26) we conclude

$$
6 R^{-1} \mathbf{M}\left(\mathbf{T}_{K}\right) \leq 2 Q \mathcal{H}^{2}\left(\mathbf{\Psi}\left(B_{r}\right)\right)+\int_{B_{r}}|D \mathcal{L}|^{2}+C \operatorname{Err}_{1}\left(\mathcal{N}, B_{r}\right)+C \operatorname{Err}_{2}\left(\mathcal{L}, B_{r}\right) .
$$

Combining the latter estimate with (3.25) we conclude the proof.

\section{HARMONIC COMPETITOR}

The most natural choice for the competitor $\mathcal{L}$ is a suitable "harmonic" extension of the boundary value $\left.\mathcal{N}\right|_{\partial B_{r}}$. Following the ideas of [3] we estimate carefully the energy of such competitor. To this purpose it is useful to introduce "polar" coordinates with center 0 in $\mathfrak{B}$ and split accordingly the Dirichlet integrand in radial and angular parts. More precisely, consider $\left(z_{0}, w_{0}\right)=\left(\left(\xi_{0}, \zeta_{0}\right), w_{0}\right) \in \partial B_{r}$ and take, locally, the standard flat coordinates $z=\left(x_{1}, x_{2}\right)$ of Definition 1.3. We then denote by $\nu$ the exterior unit vector normal to $\partial B_{r}$ at $\left(z_{0}, w_{0}\right)$ and by $\tau$ the corresponding tangent unit vector obtained by rotating $\nu$ of an angle $\pi / 2$ in the counterclockwise direction, namely

$$
\nu:=\left|z_{0}\right|^{-1}\left(\xi_{0} \frac{\partial}{\partial x_{1}}+\zeta_{0} \frac{\partial}{\partial x_{2}}\right) \quad \text { and } \quad \tau:=\left|z_{0}\right|^{-1}\left(-\zeta_{0} \frac{\partial}{\partial x_{1}}+\xi_{0} \frac{\partial}{\partial x_{2}}\right) .
$$

The directional derivatives of any (multi)function $f$ on $\mathfrak{B}$ gives then two (multi)functions

$$
D_{\nu} f=\sum_{i} \llbracket D f_{i} \cdot \nu \rrbracket \quad \text { and } \quad D_{\tau} f=\sum_{i} \llbracket D f_{i} \cdot \tau \rrbracket .
$$

The Dirichlet integrand $|D f|^{2}$ enjoys then the splitting

$$
|D f|^{2}=\left|D_{\nu} f\right|^{2}+\left|D_{\tau} f\right|^{2}
$$

For the rigorous justification of these identities see [4].

Proposition 4.1. There are constants $C>0, \sigma>0$ such that, for every $r \in(0,1)$ there exists a competitor $\mathcal{L}: B_{r} \rightarrow \mathcal{A}_{Q}\left(\mathbb{R}^{2+n}\right)$ for $\mathcal{N}$ with the following additional properties:

(i) $\operatorname{Lip}(\mathcal{L}) \leq C_{3.2}^{-1},\|\mathcal{L}\|_{0} \leq C r$.

(ii) The following estimates hold:

$$
\begin{gathered}
\int_{B_{r}}|D \overline{\mathcal{L}}|^{2} \leq C r \int_{\partial B_{r}}|D \overline{\mathcal{N}}|^{2} \leq C r \mathbf{D}^{\prime}(r), \\
\int_{B_{r}}|z|^{\gamma_{0}-1}|\boldsymbol{\eta} \circ \mathcal{L}| \leq C r^{\gamma_{0}} \int_{\partial B_{r}}|\boldsymbol{\eta} \circ \mathcal{N}|+C \mathbf{H}(r) .
\end{gathered}
$$


(iii) For every $a>0$ there exists $b_{0}>0$ such that, for all $b \in\left(0, b_{0}\right)$, the following estimate holds:

$$
(2 a+b) \int_{B_{r}}|D \overline{\mathcal{L}}|^{2} \leq r \int_{\partial B_{r}}\left|D_{\tau} \mathcal{N}\right|^{2}+\frac{a(a+b)}{r} \int_{\partial B_{r}}|\mathcal{N}|^{2}+C r^{1+\sigma} \mathbf{D}^{\prime}(r) .
$$

Using this competitor in Proposition 3.2, we then infer the following corollary.

Corollary 4.2. For every $r \in(0,1)$ the following inequality holds

$$
\mathbf{D}(r) \leq C r \mathbf{D}^{\prime}(r)+C \mathbf{H}(r)+C \mathbf{F}(r)+C \boldsymbol{m}_{0}^{1 / 2} r^{\gamma_{0}} \int_{\partial B_{r}}|\boldsymbol{\eta} \circ \mathcal{N}| .
$$

For every $a>0$ there exists $b_{0}>0$ such that, for all $b \in\left(0, b_{0}\right)$ and all $\left.r \in\right] 0,1[$

$$
\mathbf{D}(r) \leq(1+C r)\left[\frac{r}{(2 a+b)} \int_{\partial B_{r}}\left|D_{\tau} \mathcal{N}\right|^{2}+\frac{a(a+b)}{r(2 a+b)} \mathbf{H}(r)\right]+C \mathcal{E}_{Q M}(r)+C r^{1+\sigma} \mathbf{D}^{\prime}(r),
$$

with

$$
\mathcal{E}_{Q M}(r) \leq \boldsymbol{\Lambda}(r)^{\eta_{0}} \mathbf{D}(r)+\mathbf{F}(r)+\mathbf{H}(r)+\boldsymbol{m}_{0}^{1 / 2} r^{\gamma_{0}} \int_{\partial B_{r}}|\boldsymbol{\eta} \circ \mathcal{N}|
$$

Proof of Corollary 4.2. Recalling that $\mathbf{H}(r) \leq C r\|\mathcal{N}\|_{\partial B_{r}}^{2} \leq C r^{3+\gamma_{0}}$ we easily infer that $\boldsymbol{\Lambda}(r) \leq C r^{2}$ and thus the inequalities follow readily from Proposition 3.2 and Proposition 4.1 .

4.1. Proof of Proposition 4.1: Step 1. First of all we observe that it suffices to exhibit $\overline{\mathcal{L}}$, as $\mathcal{L}$ can be recovered from it via the formula (3.1). Moreover, it suffices to show the estimates with $\overline{\mathcal{N}}$ in place of $\mathcal{N}$ in the right hand side, because we obviously have $|\overline{\mathcal{N}}| \leq|\mathcal{N}|$ and $|D \overline{\mathcal{N}}| \leq|D \mathcal{N}|$. Next we wish to relate $\boldsymbol{\eta} \circ \mathcal{L}$ and $\boldsymbol{\eta} \circ \overline{\mathcal{L}}$ for two maps satisfying the relation (3.1). Note that by a simple Taylor expansion we have

$$
|\boldsymbol{\eta} \circ \mathcal{L}| \leq C|\boldsymbol{\eta} \circ \overline{\mathcal{L}}|+C \mathcal{G}(\overline{\mathcal{L}}, \boldsymbol{\eta} \circ \overline{\mathcal{L}})^{2},
$$

where the constant $C$ depends on the $C^{2}$ norm of $\Psi_{0}$. In particular we record the following conclusion:

$$
\int_{B_{r}}|z|^{\gamma_{0}-1}|\boldsymbol{\eta} \circ \mathcal{L}| \leq C \int_{B_{r}}|z|^{\gamma_{0}-1}|\boldsymbol{\eta} \circ \overline{\mathcal{L}}|+C \int_{B_{r}}|z|^{\gamma_{0}-1}|\overline{\mathcal{L}}|^{2}
$$

In this step we exhibit an "harmonic" ${ }^{1}$ competitor $\mathcal{H}$ which satisfies all the requirements of the proposition except for the Lipschitz estimate. In fact we will show that there is a

\footnotetext{
${ }^{1}$ We remark that the competitor used here does not coincide, in general, with the Dirichlet minimizer with boundary value $\left.\overline{\mathcal{N}}\right|_{\partial B_{r}}$.
} 
$W^{1,2} \operatorname{map} \mathcal{H}: B_{r} \rightarrow \mathcal{A}_{Q}\left(\mathbb{R}^{2+\bar{n}}\right)$ such that

$$
\begin{aligned}
& \left.\mathcal{H}\right|_{\partial B_{r}}=\left.\overline{\mathcal{N}}\right|_{\partial B_{r}} \quad \text { and } \quad\|\mathcal{H}\|_{L^{\infty}\left(B_{r}\right)} \leq Q\|\overline{\mathcal{N}}\|_{L^{\infty}\left(\partial B_{r}\right)} \\
& \int_{B_{r}}|D \mathcal{H}|^{2} \leq C r \int_{\partial B_{r}}|D \overline{\mathcal{N}}|^{2} \\
& \int_{B_{r}}|z|^{\gamma_{0}-1}|\boldsymbol{\eta} \circ \mathcal{H}| \leq C r^{\gamma_{0}} \int_{\partial B_{r}}|\boldsymbol{\eta} \circ \overline{\mathcal{N}}| \\
& \int_{B_{r}}|z|^{\gamma_{0}-1}|\mathcal{H}|^{2} \leq C r^{\gamma_{0}} \int_{\partial B_{r}}|\overline{\mathcal{N}}|^{2} \\
& (2 a+b) \int_{B_{r}}|D \overline{\mathcal{H}}|^{2} \leq r \int_{\partial B_{r}}\left|D_{\tau} \overline{\mathcal{N}}\right|^{2}+\frac{a(a+b)}{r} \int_{\partial B_{r}}|\overline{\mathcal{N}}|^{2} .
\end{aligned}
$$

In these estimates we do not use any of the particular properties of $\overline{\mathcal{N}}$ and indeed for any Lipschitz multivalued map $\overline{\mathcal{N}}: B_{r} \rightarrow \mathcal{A}_{Q}\left(\mathbb{R}^{2+\bar{n}}\right)$ there is such an "harmonic" competitor. Therefore, given the scaling invariance of the estimates, we will assume without loss of generality that $r=1$.

Let $D_{r}:=\{|z|<r\}$ denote the disk of radius $r$ in $\mathbb{R}^{2}$, which we identify with the complex plane. We start by defining the "winding map" W: $\bar{D}_{1} \rightarrow \mathfrak{B}$ given (in complex notation) by

$$
\mathbf{W}(z):=\left(z^{\bar{Q}}, z\right) .
$$

We then consider the multivalued map $\mathcal{U}:=\overline{\mathcal{N}} \circ \mathbf{W}$. Let $\theta \mapsto u(\theta)$ be its trace on $\partial D_{1}(0)$, which we parametrize with the angle $\theta \in[0,2 \pi]$. According to [4, Proposition 1.5] we can decompose $u$ in a superposition of simple functions $u(\theta)=\sum_{j=1}^{J} u_{j}(\theta)$ such that, for every $j=1, \ldots, J$,

$$
u_{j}(\theta)=\sum_{i=1}^{Q_{j}} \llbracket \gamma_{j}\left(\frac{\theta+2 \pi i}{Q_{j}}\right) \rrbracket,
$$

where the $\gamma_{j}:[0,2 \pi] \rightarrow \mathbb{R}^{2+\bar{n}}$ are periodic Lipschitz functions. Next consider the Fourier's expansion of each $\gamma_{j}$

$$
\gamma_{j}(\theta)=\frac{a_{j, 0}}{2}+\sum_{l=1}^{\infty}\left(a_{j, l} \cos (l \theta)+b_{j, l} \sin (l \theta)\right),
$$

and its harmonic extension, which in polar coordinates $(\rho, \theta)$ reads as

$$
\zeta_{j}(\rho, \theta):=\frac{a_{j, 0}}{2}+\sum_{l=1}^{\infty} \rho^{l}\left(a_{j, l} \cos (l \theta)+b_{j, l} \sin (l \theta)\right) .
$$

We then can define the "harmonic" competitor for $\mathcal{U}$, which is the $Q$-valued map

$$
\mathcal{V}(\rho, \theta):=\sum_{j=1}^{J} \sum_{i=1}^{Q_{j}} \llbracket \zeta_{j}\left(\rho^{1 / Q_{j}}, \frac{\theta+2 \pi i}{Q_{j}}\right) \rrbracket
$$


and the "harmonic" competitor for $\overline{\mathcal{N}}$, which is $\mathcal{H}=\mathcal{V} \circ \mathbf{W}^{-1}$. Observe that the first claim in (4.7) is obvious, whereas the second claim follows from the maximum principle for classical harmonic functions.

Simple computations and the conformality of $\mathbf{W}$, see for instance [4, Proof of Proposition $5.2]$, yield

$$
\begin{aligned}
\int_{B_{1}}|D \mathcal{H}|^{2}= & \int_{D_{1}}|D \mathcal{V}|^{2}=\pi \sum_{j=1}^{J} \sum_{l=1}^{\infty} l\left(\left|a_{j, l}\right|^{2}+\left|b_{j, l}\right|^{2}\right), \\
& \int_{\partial B_{1}}\left|D_{\tau} \mathcal{H}\right|^{2}=\frac{\pi}{\bar{Q}} \sum_{j=1}^{J} \sum_{l=1}^{\infty} \frac{l^{2}}{Q_{j}}\left(\left|a_{j, l}\right|^{2}+\left|b_{j, l}\right|^{2}\right), \\
\int_{\partial B_{1}}|\mathcal{H}|^{2}= & \pi \bar{Q} \sum_{j=1}^{J} Q_{j}\left(\frac{\left|a_{j, 0}\right|^{2}}{2}+\sum_{l=1}^{\infty}\left(\left|a_{j, l}\right|^{2}+\left|b_{j, l}\right|^{2}\right)\right) .
\end{aligned}
$$

Clearly, (4.8) follows from the first and second inequality, with the constant $C=\bar{Q} Q_{1} \leq$ $\bar{Q} Q$, assuming that $Q_{1}=\max \left\{Q_{1}, \ldots, Q_{j}\right\}$. (4.11) follows from the fact that, for any chosen $a>0$, if $b_{0}$ is sufficiently small and $0<b<b_{0}$, then

$$
(2 a+b) \ell \leq \frac{\ell^{2}}{\bar{Q} Q_{j}}+\bar{Q} Q_{j} \ell a(a+b) \quad \forall \ell \in \mathbb{N} .
$$

The latter claim is elementary and the reader can consult, for instance, Step 2 in the proof of [4, Proposition 5.2].

Observe next that $\boldsymbol{\eta} \circ \mathcal{V}$ is the classical harmonic extension of the single-valued function $\left.\boldsymbol{\eta} \circ \boldsymbol{U}\right|_{\partial D_{1}}$. We then have the classical estimates

$$
\|\boldsymbol{\eta} \circ \mathcal{V}\|_{L^{\infty}\left(D_{\left.2^{1 / \bar{Q}}\right)}\right.}+\|\boldsymbol{\eta} \circ \mathcal{V}\|_{L^{1}\left(D_{1}\right)} \leq C\|\boldsymbol{\eta} \circ \mathcal{U}\|_{L^{1}\left(\partial D_{1}\right)}
$$

In particular we conclude easily

$$
\|\boldsymbol{\eta} \circ \mathcal{H}\|_{L^{\infty}\left(B_{1 / 2}\right)}+\|\boldsymbol{\eta} \circ \mathcal{H}\|_{L^{1}\left(B_{1} \backslash B_{1 / 2}\right)} \leq C \int_{\partial B_{1}}|\boldsymbol{\eta} \circ \overline{\mathcal{N}}|
$$

because the change of variables $\mathbf{W}^{-1}$ is smooth on $B_{1} \backslash B_{1 / 2}$. The integrability of $|z|^{\gamma_{0}-1}$ on $B_{1}$ gives then

$$
\int_{B_{1}}|z|^{\gamma_{0}-1}|\boldsymbol{\eta} \circ \mathcal{H}(z, w)| d z \leq C\|\boldsymbol{\eta} \circ \mathcal{H}\|_{L^{\infty}\left(B_{1 / 2}\right)}+C\|\boldsymbol{\eta} \circ \mathcal{H}\|_{L^{1}\left(B_{1} \backslash B_{1 / 2}\right)},
$$

which in turn completes the proof of (4.9).

A similar argument proves (4.10). Using the classical theory of single valued harmonic functions we see indeed that $\left\|\zeta_{j}\right\|_{L^{2}\left(B_{1}\right)}+\left\|\zeta_{j}\right\|_{L^{\infty}\left(B_{1 / 2}\right)} \leq C\left\|\gamma_{j}\right\|_{L^{2}\left(\partial B_{1}\right)}$ and thus, using the fact that $\mathbf{W}$ is smooth on $B_{1} \backslash B_{1 / 2}$, we conclude that

$$
\|\mathcal{H}\|_{L^{\infty}\left(B_{1 / 2}\right)}^{2}+\|\mathcal{H}\|_{L^{2}\left(B_{1} \backslash B_{1 / 2}\right)}^{2} \leq C \int_{\partial B_{1}}|\overline{\mathcal{N}}|^{2} .
$$

From this we easily conclude (4.10). 
4.2. Proof of Proposition 4.1: Step 2. We keep the notation of the previous paragraphs and assume that $\overline{\mathcal{N}}$ is defined in $B_{1}$, after scaling. The specific scaling that we are using is the one which preserves the Lipschitz constant and is given by

$$
\overline{\mathcal{N}}(z, w) \mapsto r^{-1} \overline{\mathcal{N}}\left(r z, r^{1 / \bar{Q}} w\right)
$$

and by abuse of notation we keep the symbols $\overline{\mathcal{N}}, \overline{\mathcal{L}}$, etc. for all the rescaled maps.

Under this scaling we then have the estimates $\|\overline{\mathcal{N}}\|_{L^{\infty}} \leq C \boldsymbol{m}_{0}^{1 / 4} r^{\gamma_{0} / 2}$ and $\operatorname{Lip}(\overline{\mathcal{N}}) \leq$ $\Lambda(r)^{\eta_{0}}$ and we want to show that we can modify $\mathcal{H}$ to a competitor $\overline{\mathcal{L}}$ with $\operatorname{Lip}(\overline{\mathcal{L}}) \leq C_{3.2}^{-1}$, satisfying

$$
\begin{aligned}
& \left.\overline{\mathcal{L}}\right|_{\partial B_{1}}=\left.\overline{\mathcal{N}}\right|_{\partial B_{1}} \quad \text { and } \quad\|\overline{\mathcal{L}}\|_{L^{\infty}\left(B_{1}\right)} \leq C\|\overline{\mathcal{N}}\|_{L^{\infty}\left(\partial B_{1}\right)} \\
& \int_{B_{1}}|D \overline{\mathcal{L}}|^{2} \leq C\left(1+r^{\sigma}\right) \int_{B_{1}}|D \mathcal{H}|^{2}+C \boldsymbol{\Lambda}(r)^{\sigma} \int_{\partial B_{1}}|D \overline{\mathcal{N}}|^{2} \\
& \int_{B_{r}}|z|^{\gamma_{0}-1}|\overline{\mathcal{L}}|^{2} \leq C \int_{\partial B_{1}}|\overline{\mathcal{N}}|^{2} \\
& \int_{B_{1}}|z|^{\gamma_{0}-1}|\boldsymbol{\eta} \circ \overline{\mathcal{L}}| \leq C \int_{\partial B_{1}}|\boldsymbol{\eta} \circ \overline{\mathcal{N}}| .
\end{aligned}
$$

Observe that the harmonic functions $\zeta_{j}$ defined in (4.12) are Lipschitz in every ball $D_{1-t}$ for $0<t<1$ with an estimate of the form

$$
\left\|D \zeta_{j}\right\|_{L^{\infty}\left(D_{1-t}\right)} \leq \frac{C}{t} \operatorname{Lip}\left(\gamma_{j}\right) \leq \frac{C}{t} \operatorname{Lip}(\overline{\mathcal{N}}) \leq \frac{C \boldsymbol{\Lambda}(r)^{\eta_{0}}}{t} .
$$

They are not Lipschitz up to the boundary $\partial D_{1}$ because the Dirichlet to Neumann map $\gamma_{j} \rightarrow \frac{\partial \zeta_{j}}{\rho}(1, \cdot)$ does not map $L^{\infty}$ into $L^{\infty}$. However we have the estimate

$$
\left\|D \zeta_{j}\right\|_{L^{p}\left(D_{1}\right)} \leq C_{p}\left\|\gamma_{j}\right\|_{W^{1, p}\left(\partial D_{1}\right)} \leq C_{p} \boldsymbol{\Lambda}(r)^{\eta_{0}}
$$

for every $p<\infty$. In particular, we can bound

$$
\left\|\zeta_{j}(1-t, \cdot)-\gamma_{j}\right\|_{W^{1,1}\left(\partial D_{1}\right)} \leq C_{2} t^{1 / 2} \boldsymbol{\Lambda}(r)^{\eta_{0}},
$$

which in turn implies

$$
\max \left|\zeta_{j}(1-t, \theta)-\gamma_{j}(\theta)\right| \leq C_{2} t^{1 / 2} \boldsymbol{\Lambda}(r)^{\eta_{0}} .
$$

Choose $t:=\boldsymbol{\Lambda}(r)^{\eta_{0} / 2}$ and define a new map $\xi_{j}$ as

$$
\xi_{j}(\rho, \theta):= \begin{cases}\zeta_{j}(\rho, \theta) & \text { for } \rho \leq 1-t \\ \frac{1-\rho}{t} \zeta_{j}(1-t, \theta)+\frac{\rho-(1-t)}{t} \gamma_{j}(\theta) & \text { for } 1-t \leq \rho \leq 1 .\end{cases}
$$

Now, (4.20) and (4.21) imply that $\left\|D \zeta_{j}\right\| \leq C \boldsymbol{\Lambda}(r)^{\eta_{0} / 2}$. Moreover we obviously have

$$
\begin{aligned}
\int_{D_{1}}\left|D \xi_{j}\right|^{2} & \leq \int_{D_{1}}\left|D \zeta_{j}\right|^{2}+C \boldsymbol{\Lambda}(r)^{\eta_{0}}\left(\int_{\partial D_{1-t}}\left|D \zeta_{j}\right|^{2}+\int_{\partial D_{1}}\left|D \gamma_{j}\right|^{2}\right) \\
& \leq \int_{D_{1}}\left|D \zeta_{j}\right|^{2}+C r \boldsymbol{\Lambda}(r)^{\eta_{0}} \int_{\partial B_{1}}\left|D \gamma_{j}\right|^{2} .
\end{aligned}
$$


We can now define two "intermediate" maps

$$
\mathcal{V}^{0}(\rho, \theta):=\sum_{j=1}^{J} \sum_{i=1}^{Q_{j}} \llbracket \xi_{j}\left(\rho^{1 / Q_{j}}, \frac{\theta+2 \pi i}{Q_{j}}\right) \rrbracket
$$

and $\mathcal{L}^{0}:=\mathcal{V}^{0} \circ \mathbf{W}^{-1}$. It is then immediate to see that $\mathcal{L}^{0}$ enjoys the bound $\operatorname{Lip}\left(\mathcal{L}^{0}\right) \leq$ $C \boldsymbol{\Lambda}(r)^{\eta_{0} / 2}$ on the domain $B_{1} \backslash B_{1 / 4}$ and that all the estimates (4.16), (4.17) and (4.19). On the other hand the differential $D \mathcal{L}^{0}$ is singular in the origin and in fact it is rather easy to see that we have the bound

$$
\left|D \mathcal{L}^{0}(z, w)\right|^{2} \leq C|z|^{2-2 /(Q \bar{Q})} \int_{B_{1}}\left|D \mathcal{L}^{0}\right|^{2} .
$$

In order to produce $\overline{\mathcal{L}}$ we need to smooth the singularity of $\mathcal{L}^{0}$ at the origin. There are several ways to do this and we present here one possibility. First of all we fix $2<p<$ $2 Q \bar{Q} /(2 Q \bar{Q}-2)$ and observe that (4.23) yields the estimate

$$
\int_{B_{3 / 4}}\left|D \mathcal{L}^{0}(z, w)\right|^{p} \leq C\left(\int_{B_{1}}\left|D \mathcal{L}^{0}\right|^{2}\right)^{p / 2} .
$$

Next we define

$$
M\left|D \mathcal{L}^{0}(z, w)\right|:=\sup _{\rho<1 / 4} \frac{1}{\rho^{2}} \int_{B_{\rho}(z, w)}\left|D \mathcal{L}^{0}(z, w)\right|
$$

and let

$$
A:=\left\{(z, w): M\left|D \mathcal{L}^{0}(z, w)\right| \geq c_{0}\right\}
$$

where $c_{0}$ is a constant to be chosen later. Observe that, given the Lipschitz bound for $\mathcal{L}^{0}$ outside the origin, for $r$ sufficiently small the set $A$ is contained in $B_{1 / 2}$. Arguing as in the proof of [4, Proposition 4.4] we have the Lipschitz estimate $\operatorname{Lip}\left(\mathcal{L}^{0}\right) \leq C c_{0}$ on $B_{1} \backslash A$, where $C$ is a dimensional constant. We can then use the Lipschitz extension of [4, Theorem 1.7] to extend $\mathcal{L}^{0}$ to $\overline{\mathcal{L}}$ on $A$ so that $\operatorname{Lip}(\mathcal{L}) \leq C c_{0}$. Choosing $c_{0}$ accordingly we achieve the desired Lipschitz bound on $B_{1}$. As for (4.16) and (4.18) observe that the extension satisfies

$$
\|\overline{\mathcal{L}}\|_{L^{\infty}\left(B_{1 / 2}\right)}^{2} \leq C\|\mathcal{H}\|_{L^{\infty}\left(B_{3 / 4}\right)}^{2}
$$

and coincides with $\mathcal{L}_{0}$ on $B_{1} \backslash B_{1 / 2}$. As for (4.19), it would suffice to show that $|\boldsymbol{\eta} \circ \overline{\mathcal{L}}| \leq$ $C|\boldsymbol{\eta} \circ \overline{\mathcal{N}}|$. This can be easily achieved in the following way: we make a Lipschitz extension of $\mathcal{L}^{0}$, subtract from each sheet the average and then sum back to each sheet a Lipschitz extension of $\boldsymbol{\eta} \circ \mathcal{L}^{0}$.

As for (4.17) we compute

$$
\begin{aligned}
\int|D \overline{\mathcal{L}}|^{2} & \leq \int\left|D \mathcal{L}^{0}\right|^{2}+C c_{0}^{2}|A| \leq \int\left|D \mathcal{L}^{0}\right|^{2}+C c_{0}^{2-p} \int_{B_{3 / 4}}\left|D \mathcal{L}^{0}\right|^{p} \\
& \leq \int\left|D \mathcal{L}^{0}\right|^{2}\left(1+C c_{0}^{2-p}\left(\int\left|D \mathcal{L}^{0}\right|^{2}\right)^{p / 2-1}\right) .
\end{aligned}
$$


Observe that $p / 2-1>0$ and that by (4.22) and (4.8)

$$
\int\left|D \mathcal{L}^{0}\right|^{2} \leq \int|D \mathcal{H}|^{2}+C \boldsymbol{\Lambda}(r)^{\sigma / 2} \int_{\partial B_{1}}|D \overline{\mathcal{N}}|^{2} \leq C \int_{\partial B_{1}}|D \overline{\mathcal{N}}|^{2} \leq C r^{\sigma}
$$

so that

$$
\int_{B_{1}}|D \overline{\mathcal{L}}|^{2} \leq\left(1+C r^{\sigma}\right) \int_{B_{1}}|D \mathcal{H}|^{2}+C r^{\sigma} \int_{\partial B_{1}}|D \overline{\mathcal{N}}|^{2} \stackrel{(4.8)}{\leq} \int_{B_{1}}|D \mathcal{H}|^{2}+C r^{\sigma} \int_{\partial B_{1}}|D \overline{\mathcal{N}}|^{2}
$$

\section{OUTER VARIATIONS AND THE POINCARÉ INEQUALITY}

In this section we begin to exploit the first variations of the area functional on $T$ in conjunction with the estimates of the previous section. The main conclusion will be the following Poincaré inequality:

Theorem 5.1 (Poincaré inequality). There exists a constant $C_{5.1}>0$ such that if $r$ is sufficiently small, then

$$
\mathbf{H}(r) \leq C_{5.1} r \mathbf{D}(r)
$$

We record however the two main tools used to prove Theorem 5.1, since they will be useful in the future. The first one is an elementary computation. In order to state it we introduce the quantity

$$
\mathbf{E}(r):=\int_{\partial B_{r}} \sum_{j=1}^{Q}\left\langle\mathcal{N}_{j}, D_{\nu} \mathcal{N}_{j}\right\rangle
$$

Lemma 5.2. $\mathbf{H}$ is a Lipschitz function and the following identity holds for a.e. $r \in(0,1)$

$$
\mathbf{H}^{\prime}(r)=\frac{\mathbf{H}(r)}{r}+2 \mathbf{E}(r) .
$$

The second identity is a consequence of the first variations of $T$ under specific vector fields, which we call "outer variations": such variations "stretch" the normal bundle of $\mathcal{M}$ suitably and they are defined using the map $\mathcal{N}$. In the case of semicalibrated currents it is convenient to modify the Dirichlet energy suitably to gain a new quantity which enjoys better estimates. Thus, from now on $\boldsymbol{\Omega}$ will denote $\mathbf{D}$ in the cases (a) and (c) of Definition 0.1 , whereas in the case (b) it will be given by

$$
\begin{aligned}
\boldsymbol{\Omega}(r) & :=\mathbf{D}(r)+\mathbf{L}(r) \\
& :=\mathbf{D}(r)+\int_{\boldsymbol{\Psi}\left(B_{r}\right)} \sum_{i=1}^{Q}\left\langle\xi_{1}(p) \wedge D_{\xi_{2}} N_{i}(p) \wedge N_{i}(p)+D_{\xi_{1}} N_{i}(p) \wedge \xi_{2}(p) \wedge N_{i}(p), d \omega(p)\right\rangle d p .
\end{aligned}
$$

Proposition 5.3 (Outer variations). There exist constants $C_{5.3}>0$ and $\kappa>0$ such that, if $r>0$ is small enough, then the inequality

$$
|\Omega(r)-\mathbf{E}(r)| \leq C_{5.3} \mathcal{E}_{O V}(r)
$$


holds with

$$
\mathcal{E}_{O V}(r)=\boldsymbol{\Lambda}(r)^{\kappa}\left(\mathbf{D}(r)+\frac{\mathbf{H}(r)}{r}+r \mathbf{D}^{\prime}(r)\right)+\mathbf{F}(r)+r^{1+\gamma_{0}} \frac{d}{d r}\left\|T-\mathbf{T}_{F}\right\|\left(\mathbf{p}^{-1}\left(\mathbf{\Psi}\left(B_{r}\right)\right)\right) .
$$

Moreover

$$
|\mathbf{L}(r)| \leq C \boldsymbol{m}_{0}^{1 / 2} r^{2-\gamma_{0}} \mathbf{D}(r)+C \boldsymbol{m}_{0}^{1 / 2} \mathbf{F}(r) .
$$

5.1. Proof of Lemma 5.2. The Lipschitz regularity of $\mathbf{H}$ follows from the Lipschitz regularity of $\mathcal{N}$. Consider next the map $i_{r}: \mathfrak{B} \rightarrow \mathfrak{B}$ given by $i_{r}(z, w)=\left(r z, r^{1 / \bar{Q}} w\right)$. By a simple change of variables we compute

$$
\mathbf{H}(r)=\int_{\partial B_{1}}|\mathcal{N}|^{2}\left(i_{r}\left(z^{\prime}, w^{\prime}\right)\right) r
$$

The formula (5.3) is then an elementary computation using the chain rule for multifunctions, cf. [4].

5.2. Proof of Proposition 5.3. The inequality (5.6) is a simple consequence of

$$
|\mathbf{L}(r)| \leq C \boldsymbol{m}_{0}^{1 / 2} \int_{B_{r}}|D \mathcal{N}||\mathcal{N}| \leq C \boldsymbol{m}_{0}^{1 / 2} \int_{B_{r}}|z|^{2-\gamma_{0}}|D \mathcal{N}|^{2}+C \boldsymbol{m}_{0}^{1 / 2} \int_{B_{r}}|z|^{\gamma_{0}-2}|\mathcal{N}|^{2}
$$

In order to show (5.4) we fix a test function $\phi \in C_{c}^{\infty}(\mathbb{R})$, nonnegative, symmetric, with support in $]-1,1[$ and monotone decreasing on $[0,1]$. We then follow [8, Section 3.3] and, having fixed $r$, we define the vector field $X^{o}$ on $\mathbf{V}_{u, a}$ via

$$
X^{o}(p):=\varphi(\mathbf{p}(p))(p-\mathbf{p}(p)) \quad \text { where } \quad \varphi(\mathbf{\Psi}(z, w))=\phi\left(\frac{|z|}{r}\right) .
$$

For $r$ small enough, by (2.13) we can argue as in [8, Section 3.3] and deduce via the change of coordinates given by $\boldsymbol{\Psi}$, that

$$
\delta \mathbf{T}_{F}(X)=\int_{\mathfrak{B}} \phi\left(\frac{|z|}{r}\right)|D \mathcal{N}|^{2}+r^{-1} \int_{\mathfrak{B}} \phi^{\prime}\left(\frac{|z|}{r}\right) \sum_{j=1}^{Q}\left\langle\mathcal{N}_{j}, D_{\nu} \mathcal{N}_{j}\right\rangle+\sum_{i=1}^{3} \operatorname{Err}_{i}^{o}
$$

with

$$
\begin{gathered}
\operatorname{Err}_{1}^{o}=\left|\int_{\mathcal{M}} \varphi\left\langle H_{\mathcal{M}}, \boldsymbol{\eta} \circ N\right\rangle\right| \leq C \boldsymbol{m}_{0}^{1 / 2} \int_{B_{r}}|z|^{\gamma_{0}-1}|\boldsymbol{\eta} \circ \mathcal{N}| \stackrel{(2.14)}{\leq} C \boldsymbol{\Lambda}^{\eta_{0}}(r) \mathbf{D}(r)+C \mathbf{F}(r) \\
\operatorname{Err}_{2}^{o} \leq C \int_{\mathcal{M}}|\varphi|\left|A_{\mathcal{M}}\right|^{2}|N|^{2} \leq C \mathbf{F}(r)
\end{gathered}
$$




$$
\begin{gathered}
\operatorname{Err}_{3}^{o} \leq C \int_{\mathcal{M}}\left(|\varphi|\left(|D N|^{2}|N|\left|A_{\mathcal{M}}\right|+|D N|^{4}\right)+|D \varphi|\left(|D N|^{3}|N|+|D N||N|^{2}\left|A_{\mathcal{M}}\right|\right)\right) \\
\leq C \int_{B_{r}}\left[\left(\frac{|\mathcal{N}|^{2}}{|z|^{2-2 \gamma_{0}}}+|D \mathcal{N}|^{4}\right)-r^{-1} \phi^{\prime}\left(\frac{|z|}{r}\right) r^{1+\gamma_{0}}|D \mathcal{N}|^{3}-r^{-1} \phi^{\prime}\left(\frac{|z|}{r}\right)|D \mathcal{N}| \frac{|\mathcal{N}|^{2}}{|z|^{1-\gamma_{0}}}\right] \\
\stackrel{(2.13) \&(2.12)}{\leq} C \boldsymbol{\Lambda}^{\eta_{0}}(r) \mathbf{D}(r)+C \mathbf{F}(r)-C \boldsymbol{\Lambda}(r)^{\eta_{0}} \int_{B_{r}} r^{-1} \phi^{\prime}\left(\frac{|z|}{r}\right) \frac{|\mathcal{N}|^{2}}{|z|^{1-\gamma_{0}}} \\
-C r^{1+\gamma_{0}} \Lambda^{\eta_{0}} \int_{B_{r}} r^{-1} \phi^{\prime}\left(\frac{|z|}{r}\right)|D \mathcal{N}|^{2}
\end{gathered}
$$

(We recall that $\phi^{\prime} \leq 0$ on $\left.[0,1]\right)$ ).

We next drop the superscript from $X^{o}$ and we distinguish two situations:

- In the cases (a) and (c) of Definition 0.1, we denote by $X^{\perp}$ and $X^{T}$ the projections of $X$ on the normal and the tangential bundle of $\Sigma$, respectively. Then $\delta T\left(X^{T}\right)=0$ and therefore

$$
\left|\delta \mathbf{T}_{F}(X)\right| \leq \underbrace{\left|\delta \mathbf{T}_{F}(X)-\delta T(X)\right|}_{\text {Err }_{4}^{o}}+\underbrace{\left|\delta T\left(X^{\perp}\right)\right|}_{\text {Erro }_{5}^{o}}
$$

- In case (b), since $\delta T(X)=T(d w\lrcorner X)$, we estimate

$$
\left.\mid \delta \mathbf{T}_{F}(X)-\mathbf{T}_{F}(d \omega\lrcorner X\right) \mid \leq \underbrace{\left.\left.\left|\delta \mathbf{T}_{F}(X)-\delta T(X)\right|+\mid T(d \omega\lrcorner X\right)-\mathbf{T}_{F}(d \omega\lrcorner X\right) \mid}_{\text {Err }_{4}^{o}} .
$$

In both cases we have

$$
\begin{aligned}
\operatorname{Err}_{4}^{o} \leq Q & \int_{\operatorname{spt}(T) \backslash \operatorname{Im}(F)}\left|\operatorname{div}_{\vec{T}} X\right| d\|T\|+Q \int_{\operatorname{Im}(F) \backslash \operatorname{spt}(T)}\left|\operatorname{div}_{\overrightarrow{\mathbf{T}}_{F}} X\right| d\left\|\mathbf{T}_{F}\right\| \\
& +Q\|d \omega\|_{\infty} \int|X| d\left\|T-\mathbf{T}_{F}\right\|,
\end{aligned}
$$

where we use the convention that $\omega=0$ in the cases (a) and (c). We then can estimate

$$
\begin{aligned}
\operatorname{Err}_{4}^{o} \leq C & \int\left(\varphi^{\prime}(\mathbf{p}(p))|p-\mathbf{p}(p)|+\varphi(\mathbf{p}(p))\right) d\left\|T-\mathbf{T}_{F}\right\| \\
& \stackrel{(2.12) \&(2.15)}{\leq} C \boldsymbol{\Lambda}^{\eta_{0}}(r) \mathbf{D}(r)+C \mathbf{F}(r)+C r^{1+\gamma_{0}} \underbrace{\int|\nabla \varphi(\mathbf{p}(p))||p-\mathbf{p}(p)| d\left\|T-\mathbf{T}_{F}\right\|}_{S(\varphi)} .
\end{aligned}
$$

In case (b) we have that

$$
\left.\mathbf{T}_{F}(d \omega\lrcorner X\right)=\int_{\mathcal{M}} \varphi \sum_{i=1}^{Q}\left\langle\left(\xi_{1}+D_{\xi_{1}} N_{i}\right) \wedge\left(\xi_{2}+D_{\xi_{2}} N_{i} \cdot \xi_{2}\right) \wedge N_{i}, d \omega\left(p+N_{i}(p)\right) .\right.
$$


Clearly

$$
\begin{aligned}
& \left.\quad \mid \mathbf{T}_{F}(d \omega\lrcorner X\right)-\int_{\mathcal{M}} \varphi \sum_{i=1}^{Q}\left\langle\left(\xi_{1}+D_{\xi_{1}} N_{i}\right) \wedge\left(\xi_{2}+D_{\xi_{2}} N_{i} \cdot \xi_{2}\right) \wedge N_{i}, d \omega(p)\right\rangle \mid \\
& \leq C\|d \omega\|_{1} \int \varphi|N|^{2}
\end{aligned}
$$

and we can therefore conclude

$$
\begin{aligned}
& \left.\mid \mathbf{T}_{F}(d \omega\lrcorner X\right)-\int_{\mathcal{M}} \varphi \sum_{i=1}^{Q}\left\langle\xi_{1}(p) \wedge D_{\xi_{2}} N_{i}(p) \wedge N_{i}(p)+D_{\xi_{1}} N_{i}(p) \wedge \xi_{2}(p) \wedge N_{i}(p), d \omega(p)\right\rangle \mid \\
\leq & C\|d \omega\|_{0} \int \varphi|N|\left\|\left.D N\right|^{2}+C\right\| d \omega\left\|_{0} \int \varphi|\boldsymbol{\eta} \circ N|+C\right\| d \omega \|_{1} \int \varphi|N|^{2} .
\end{aligned}
$$

Letting $\phi$ converge to the characteristic function of the interval $[-1,1]$, we reach the conclusion (5.4). The only term which needs some care is the term $S(\varphi)$ in (5.11). Note that we can approximate the characterstic function of $[-1,1]$ with an increasing sequence of functions $\phi_{j}$ with the property that $\left|\phi_{j}^{\prime}\right| \leq C j, 0 \leq \phi_{j} \leq 1$ and $\phi_{j} \equiv 1$ on $[-1+1 / j, 1-1 / j]$. Then we would have

$$
\underset{j}{\limsup } S\left(\varphi_{j}\right) \leq C \limsup _{j} \frac{j}{r}\left\|T-\mathbf{T}_{F}\right\|\left(\mathbf{\Psi}\left(B_{r} \backslash B_{r(1-1 / j)}\right)\right) \leq C \frac{d}{d r}\left\|T-\mathbf{T}_{F}\right\|\left(\mathbf{\Psi}\left(B_{r}\right)\right),
$$

by the monotonicity of the function $r \mapsto\left\|T-\mathbf{T}_{F}\right\|\left(\Psi\left(B_{r}\right)\right)$.

In the cases (a) and (c) we follow the same argument, but we need to bound the additional term $\operatorname{Err}_{5}^{o}$. In order to deal with the latter term we argue as in [8, Section 4.1]. In particular we bound

$$
\begin{aligned}
\operatorname{Err}_{5}^{o} \leq & \left|\int \operatorname{div}_{\vec{T}} X^{\perp} d\|T\|\right| \\
\leq & \underbrace{\int_{\operatorname{spt}(T) \backslash \operatorname{Im}(F)}\left|\operatorname{div}_{\vec{T}} X\right| d\|T\|+\int_{\operatorname{Im}(F) \backslash \operatorname{spt}(T)}\left|\operatorname{div}_{\overrightarrow{\mathbf{T}}_{F}} X\right| d\left\|\mathbf{T}_{F}\right\|}_{I_{1}} \\
& +\underbrace{\left|\int\left\langle X^{\perp}, h\left(\overrightarrow{\mathbf{T}}_{F}(p)\right)\right\rangle d\left\|\mathbf{T}_{F}\right\|\right|}_{I_{2}},
\end{aligned}
$$

where $h\left(v_{1} \wedge v_{2}\right):=\sum_{i=1}^{2} A_{\Sigma}\left(v_{i}, v_{i}\right)$. Since the projection on the normal to $\Sigma$ is a $C^{2, \varepsilon_{0}}$ map, $X^{\perp}$ enjoys the same $C^{1}$ bounds as $X$ and $I_{1}$ can be controlled as $\operatorname{Err}_{4}^{o}$. The term $I_{2}$ can be estimated using

$$
\left|X^{o \perp}(p)\right|=\varphi\left|\mathbf{p}_{T_{p} \Sigma^{\perp}}(p-\mathbf{p}(p))\right| \leq C \mathbf{c}(\Sigma) \varphi|p-\mathbf{p}(p)|^{2} \leq C \boldsymbol{m}_{0}^{1 / 2} \varphi|p-\mathbf{p}(p)|^{2} \quad \forall p \in \Sigma .
$$

In particular we achieve $I_{2} \leq C \mathbf{H}(r)$, which concludes the proof. 
5.3. Proof of Theorem 5.1. In order to prove the theorem we start estimating the error term $\mathbf{F}$.

Lemma 5.4. There exist a constant $C_{5.4}>0$ (depending on $\gamma_{0}$ ) such that

$$
\mathbf{F}(r) \leq C_{5.4} r^{\gamma_{0}-1} \mathbf{H}(r)+C_{5.4} r^{\gamma_{0}} \mathbf{D}(r) \quad \forall r \in(0,1) .
$$

Proof. Using (5.3) and an integration by parts we infer that

$$
\gamma_{0} \int_{0}^{r} \frac{\mathbf{H}(\rho)}{\rho^{2-\gamma_{0}}} d \rho=\left.\frac{\mathbf{H}(\rho)}{\rho^{1-\gamma_{0}}}\right|_{0} ^{r}-\int_{0}^{r} \frac{d}{d \rho}\left(\frac{\mathbf{H}(\rho)}{\rho}\right) \rho^{\gamma_{0}} d \rho=\frac{\mathbf{H}(r)}{r^{1-\gamma_{0}}}-\int_{0}^{r} \frac{2 \mathbf{E}(\rho)}{\rho^{1-\gamma_{0}}} d \rho .
$$

The Cauchy-Schwarz inequality yields then the following bound for every $\varepsilon$ :

$$
|\mathbf{E}(r)| \leq \frac{\varepsilon}{r} \int_{\partial B_{r}}|\mathcal{N}|^{2}+\frac{r}{4 \varepsilon} \int_{\partial B_{r}}|D \mathcal{N}|^{2}=\varepsilon \frac{\mathbf{H}(r)}{r}+\frac{r \mathbf{D}^{\prime}(r)}{4 \varepsilon} .
$$

Therefore, by choosing $\varepsilon=\gamma_{0} / 2$, we deduce (5.13) from (5.14) and (5.15).

Proof of Theorem 5.1. In view of Lemma 5.4, for $r$ sufficiently small, the almost minimizing condition (4.4) reads as

$$
\mathbf{D}(r) \leq C r \mathbf{D}^{\prime}(r)+C \frac{\mathbf{H}(r)}{r^{1-\gamma_{0}}}+C \boldsymbol{m}_{0}^{1 / 2} r^{\gamma_{0}} \int_{\partial B_{r}}|\boldsymbol{\eta} \circ \mathcal{N}| .
$$

Dividing by the radius and integrating we get

$$
\begin{aligned}
\int_{0}^{r} \frac{\mathbf{D}(s)}{s} d s & \leq C \int_{0}^{r}\left(\mathbf{D}^{\prime}(\rho)+\frac{\mathbf{H}(\rho)}{\rho^{2-\gamma_{0}}}+\rho^{\gamma_{0}-1} \int_{\partial B_{\rho}}|\boldsymbol{\eta} \circ \mathcal{N}|\right) d \rho \\
& \stackrel{(5.13)}{\leq} C \mathbf{D}(r)+C \mathbf{F}(r)+C \boldsymbol{m}_{0}^{1 / 2} \int_{B_{r}} \frac{|\boldsymbol{\eta} \circ \mathcal{N}|}{|z|^{1-\gamma_{0}}} \\
& \stackrel{(2.14)}{\leq} C \mathbf{D}(r)+C\left(\boldsymbol{\Lambda}^{\eta_{0}}(r) \mathbf{D}(r)+\mathbf{F}(r)\right) \leq C \mathbf{D}(r)+C r^{\gamma_{0}-1} \mathbf{H}(r) .
\end{aligned}
$$

Therefore, using Lemma 5.2 we deduce that

$$
\begin{aligned}
\frac{\mathbf{H}(r)}{r}= & \int_{0}^{r} \frac{2 \mathbf{E}(\rho)}{\rho} d t \stackrel{(5.4)}{\leq} C \int_{0}^{r} \frac{\mathbf{D}(\rho)}{\rho} d \rho \\
& +C \int_{0}^{r}\left(\frac{\mathbf{H}(\rho)}{\rho^{2-2 \gamma_{0}}}+\rho^{\gamma_{0}} \mathbf{D}^{\prime}(\rho)+\rho^{\gamma_{0}} \frac{d}{d \rho}\left\|T-\mathbf{T}_{F}\right\|\left(\mathbf{p}^{-1}\left(\mathbf{\Psi}\left(B_{\rho}\right)\right)\right)\right) d \rho \\
& \stackrel{(5.16)}{\leq} C \mathbf{D}(r)+C \frac{\mathbf{H}(r)}{r^{1-\gamma_{0}}}+C r^{\gamma_{0}} \mathbf{D}(r)+C \mathbf{F}(r)+C r^{\gamma_{0}}\left\|T-\mathbf{T}_{F}\right\|\left(\mathbf{p}^{-1}\left(\mathbf{\Psi}\left(B_{r}\right)\right)\right) \\
& \stackrel{(2.15) \&(5.13)}{\leq} C \mathbf{D}(r)+C \frac{\mathbf{H}(r)}{r^{1-\gamma_{0}}} .
\end{aligned}
$$

For $r$ sufficiently small this concludes the proof. 


\section{INNER VARIATIONS AND KEY ESTIMATES}

Using the Poincaré inequality in Theorem 5.1, we can give very simple estimates of the error terms in the "inner variations" of the current $T$. The latter corresponds to deformations of $T$ along appropriate vector fields which are tangent to $\mathcal{M}$. In order to state our main conclusion we need to introduce yet another quantity

$$
\mathbf{G}(r):=\int_{\partial B_{r}}\left|D_{\nu} \mathcal{N}\right|^{2}
$$

Proposition 6.1 (Inner Variations). There exist constants $C_{6.1}>0$ and $\eta>0$ such that, if $r>0$ is small enough, than the following holds

$$
\left|\mathbf{D}^{\prime}(r)-2 \mathbf{G}(r)\right| \leq C \mathcal{E}_{I V}(r)
$$

where

$$
\begin{aligned}
\mathcal{E}_{I V}(r) & =r^{2 \eta-1} \mathbf{D}(r)+\mathbf{D}(r)^{\eta} \mathbf{D}^{\prime}(r)+\frac{\boldsymbol{m}_{0}^{1 / 2}}{r^{1-\gamma_{0}}} \int_{\partial B_{r}}|\boldsymbol{\eta} \circ \mathcal{N}(z, w)| \\
& +\frac{d}{d r}\left\|T-\mathbf{T}_{F}\right\|\left(\mathbf{p}^{-1}\left(\mathbf{\Psi}\left(B_{r}\right)\right)\right) .
\end{aligned}
$$

For further use we summarize in the next lemma a set of inequalities which will be used in the next sections and which are direct consequences of all the conclusions derived so far

Lemma 6.2. There exist constant $C_{6.2}>0$ and $\eta>0$ such that for every $r$ sufficiently small the following holds:

$$
\begin{aligned}
\mathbf{F}(r)+r \mathbf{F}^{\prime}(r) & \leq C_{6.2} r^{\gamma_{0}} \mathbf{D}(r) \\
|\mathbf{L}(r)| & \leq C_{6.2} r \mathbf{D}(r) \\
\left|\mathbf{L}^{\prime}(r)\right| & \leq C_{6.2}\left(\mathbf{H}(r) \mathbf{D}^{\prime}(r)\right)^{1 / 2} \\
\mathcal{E}_{O V} & \leq C_{6.2} \mathbf{D}^{1+\eta}(r)+C_{6.2} \mathbf{F}(r)+C_{6.2} r \mathbf{D}^{\eta}(r) \mathbf{D}^{\prime}(r)+C_{6.2} r \mathcal{E}_{B P}(r), \\
\mathcal{E}_{I V}(r) & \leq C_{6.2} r^{2 \eta-1} \mathbf{D}(r)+C_{6.2} \mathbf{D}(r)^{\eta} \mathbf{D}^{\prime}(r)+C_{6.2} \mathcal{E}_{B P}(r),
\end{aligned}
$$

where

$$
\mathcal{E}_{B P}(r):=\frac{\boldsymbol{m}_{0}^{1 / 2}}{r^{1-\gamma_{0}}} \int_{\partial B_{r}}|\boldsymbol{\eta} \circ \mathcal{N}|+\frac{d}{d r}\left\|T-\mathbf{T}_{F}\right\|\left(\mathbf{p}^{-1}\left(\mathbf{\Psi}\left(B_{r}\right)\right)\right)
$$

Moreover, for every $a>0$ there exist constants $b_{0}(a), C(a)>0$ such that

$$
\mathbf{D}(r) \leq \frac{r \mathbf{D}^{\prime}(r)}{2(2 a+b)}+\frac{a(a+b) \mathbf{H}(r)}{r(2 a+b)}+C(a) r \mathcal{E}_{I V}(r) \quad \forall b<b_{0}(a) .
$$

An important corollary of the previous lemma is the following

Corollary 6.3. There exists a constant $C_{6.3}>0$ such that, if $\eta$ is the constant of Lemma 6.2, then for every $0 \leq \gamma<\eta$ and $r$ sufficiently small, the nonnegative functions $\frac{\mathcal{E}_{I V}(r)}{r^{\gamma} \mathbf{D}(r)}$ 
$\frac{\mathcal{E}_{O V}(r)}{r^{1+\gamma} \mathbf{D}(r)}$ are both integrable. Moreover, if we define the functions

$$
\begin{aligned}
\boldsymbol{\Sigma}_{I V}(r) & :=\int_{0}^{r} \frac{\mathcal{E}_{I V}(s)}{s^{\gamma} \mathbf{D}(s)} d s, \\
\boldsymbol{\Sigma}_{O V}(r) & :=\int_{0}^{r} \frac{\mathcal{E}_{O V}(s)}{s^{\gamma} \mathbf{D}(s)} d s, \\
\boldsymbol{\Sigma}(r) & :=\boldsymbol{\Sigma}_{I V}(r)+\boldsymbol{\Sigma}_{O V}(r),
\end{aligned}
$$

then

$$
\Sigma(r) \leq C_{6.3} r^{\eta-\gamma}
$$

6.1. Proof of Proposition 6.1. We evaluate the first variation of $T$ along a suitably defined vector field $X$. To this aim we fix a function $\phi \in C_{c}^{\infty}(]-1,1[)$, symmetric, nonnegative and identically one on $]-1+1 / j, 1-1 / j\left[\right.$ and with the property that $\left|\phi^{\prime}\right| \leq C j$. Then we introduce the vector field $Y: \mathcal{M} \rightarrow \mathbb{R}^{n+2}$ defined, for every $(z, w) \in \mathfrak{B} \backslash\{0\}$, by

$$
Y(\boldsymbol{\Psi}(z, w)):=\frac{|z|}{r} \phi\left(\frac{|z|}{r}\right) D_{\nu} \boldsymbol{\Psi}(z, w) \in T_{\boldsymbol{\Psi}(z, w)} \mathcal{M},
$$

and extended to be 0 at the origin.

Next we define the vector field $X_{i}: \mathbf{V}_{a, u} \rightarrow \mathbb{R}^{n+2}$ by $X_{i}(p):=Y(\mathbf{p}(p))$. Note that $X_{i}$ is the infinitesimal generator of a one parameter family of diffeomorphisms $\Phi_{\varepsilon}$ defined as $\Phi_{\varepsilon}(p):=\Gamma_{\varepsilon}(\mathbf{p}(p))+p-\mathbf{p}(p)$, where $\Gamma_{\varepsilon}$ is the one-parameter family of biLipschitz homeomorphisms of $\mathcal{M}$ generated by $Y$. In fact, since $\Gamma_{\varepsilon}$ fixes the origin, we can consider it as a $C^{2, \gamma_{0}}$ map of $\mathcal{M} \backslash\{0\}$ onto itself. Note moreover that $X_{i}$ is Lipschitz on the entire $\mathfrak{B}$.

Observe that, by Lemma 5.4 and the Poincaré inequality, $\mathbf{F}(r) \leq C r^{\gamma_{0}} \mathbf{D}(r)$, so that $\Lambda(r) \leq C \mathbf{D}(r)$. Moreover,

$$
\left|D_{\mathcal{M}} Y\right|(\Psi(z, w))+\left|\operatorname{div}_{\mathcal{M}} Y\right|(\Psi(z, w)) \leq-C r^{-2}|z| \phi^{\prime}\left(\frac{|z|}{r}\right)+C r^{-1} \phi\left(\frac{|z|}{r}\right),
$$

where we recall that $\phi^{\prime} \leq 0$ on $[0,1]$.

If $r$ is small enough, by (2.13) we can argue as in [8, Section 3.3] and deduce that

$$
\frac{1}{2}\left|\int_{\mathcal{M}}\left(|D N|^{2} \operatorname{div}_{\mathcal{M}} Y-2 \sum_{i=1}^{Q}\left\langle D N_{i}:\left(D N_{i} \cdot D_{\mathcal{M}} Y\right)\right\rangle\right)\right| \leq \sum_{k=1}^{5} \operatorname{Err}_{k}^{i},
$$

where the error terms can be bounded in the following manner.

First of all,

$$
\begin{aligned}
\operatorname{Err}_{1}^{i} & =Q\left|\int_{\mathcal{M}}\left(\left\langle H_{\mathcal{M}}, \boldsymbol{\eta} \circ N\right\rangle \operatorname{div}_{\mathcal{M}} Y+\left\langle D_{Y} H_{\mathcal{M}}, \boldsymbol{\eta} \circ N\right\rangle\right)\right| \\
& \leq C r^{-1} \boldsymbol{m}_{0}^{1 / 2} \int_{\mathfrak{B}}\left(\phi\left(\frac{|z|}{r}\right)|z|^{\gamma_{0}-1}|\boldsymbol{\eta} \circ \mathcal{N}(z, w)|-\phi^{\prime}\left(\frac{|z|}{r}\right)|z|^{\gamma_{0}-1}|\boldsymbol{\eta} \circ \mathcal{N}(z, w)|\right) \\
& \stackrel{(2.14)}{\leq} C r^{-1} \mathbf{D}^{1+\eta}(r)-C \boldsymbol{m}_{0}^{1 / 2} r^{\gamma_{0}-1} \int_{B_{r}} r^{-1} \phi^{\prime}\left(\frac{|z|}{r}\right)|\boldsymbol{\eta} \circ \mathcal{N}(z, w)|,
\end{aligned}
$$


where in the first inequality we used (6.14) and the fact that

$$
\left\langle D_{Y} H_{\mathcal{M}}, \boldsymbol{\eta} \circ N\right\rangle \leq|Y|\left|D H_{\mathcal{M}}\right||\boldsymbol{\eta} \circ N| \leq C \frac{|z|}{r} \phi\left(\frac{|z|}{r}\right)|z|^{\gamma_{0}-2}|\boldsymbol{\eta} \circ \mathcal{N}| .
$$

As for $\operatorname{Err}_{2}^{i}$ and $\operatorname{Err}_{i}^{3}$ we have

$$
\begin{aligned}
\operatorname{Err}_{2}^{i} & =C \int_{\mathcal{M}}\left|A_{\mathcal{M}}\right|^{2}\left(|D Y||N|^{2}+|Y||N||D N|\right) \\
& \leq C \boldsymbol{m}_{0} \int_{\mathfrak{B}}\left[r^{-1}\left(-\frac{|z|}{r} \phi^{\prime}\left(\left(\frac{|z|}{r}\right)\right)+\phi\left(\frac{|z|}{r}\right)\right) \frac{|\mathcal{N}|^{2}}{|z|^{2-2 \gamma_{0}}}+\frac{|z|}{r} \phi\left(\frac{|z|}{r^{2}}\right) \frac{|\mathcal{N}||D \mathcal{N}|}{|z|^{2-2 \gamma_{0}}}\right] \\
& \leq C \boldsymbol{m}_{0} r^{\gamma_{0}-1} \mathbf{D}(r)-C r^{-1} \int_{B_{r}} r^{-1} \phi^{\prime}\left(\frac{|z|}{r}\right) \frac{|\mathcal{N}|^{2}}{|z|^{1-\gamma_{0}}}
\end{aligned}
$$

and

$$
\begin{aligned}
\operatorname{Err}_{3}^{i} & \leq C \int_{\mathcal{M}}\left(|Y|\left|A_{\mathcal{M}}\right||D N|^{2}(|N|+|D N|)+|D Y|\left(\left|A_{\mathcal{M}}\right||D N||N|^{2}+|D N|^{4}\right)\right) \\
& \leq C r^{\gamma_{0}-1} \mathbf{D}(r)-C \mathbf{D}(r)^{\eta} \int_{\mathfrak{B}} r^{-1} \phi^{\prime}\left(\frac{|z|}{r}\right)|D \mathcal{N}|^{2}+C r^{-1} \mathbf{D}(r)^{\eta} \int_{\mathfrak{B}} r^{-1} \phi\left(\frac{|z|}{r}\right) \frac{|\mathcal{N}|^{2}}{|z|^{2-\gamma_{0}}} .
\end{aligned}
$$

The errors $\operatorname{Err}_{4}^{i}$ and $\operatorname{Err}_{5}^{i}$ are the same as $\operatorname{Err}_{4}^{o}$ and $\operatorname{Err}_{5}^{o}$ respectively, in Section 5.2, evaluated along a different vector field. Proceeding in the same way as in the estimate of $\operatorname{Err}_{4}^{o}$, we deduce

$$
\begin{aligned}
\operatorname{Err}_{4}^{i} & =\int_{\operatorname{spt}(T) \backslash \operatorname{Im}(F)}\left|\operatorname{div}_{\vec{T}} X_{i}\right| d\|T\|+\int_{\operatorname{Im}(F) \backslash \operatorname{spt}(T)}\left|\operatorname{div}_{\overrightarrow{\mathbf{T}}_{F}} X_{i}\right| d\left\|\mathbf{T}_{F}\right\| \\
& \leq C r^{\gamma_{0}-1} \mathbf{D}(r)+C \underbrace{\int \alpha d\left\|T-\mathbf{T}_{F}\right\|}_{S(\phi)} .
\end{aligned}
$$

where $\alpha(p)=\varphi(\mathbf{p}(p))$ and $\varphi(\boldsymbol{\Psi}(z, w))=r^{-2}|z| \phi\left(r^{-1}|z|\right)-r^{-1} \phi^{\prime}\left(r^{-1}|z|\right)$. In particular using (2.15) and the fact that $-\phi^{\prime} \leq C j$ on $[0,1]$, we infer

$$
S(\phi) \leq C r^{\gamma_{0}-1} \mathbf{D}(r)+C \frac{j}{r}\left\|T-\mathbf{T}_{F}\right\|\left(\mathbf{p}^{-1}\left(\mathbf{\Psi}\left(B_{r} \backslash B_{r(1-1 / j)}\right)\right) .\right.
$$

As for $\operatorname{Err}_{i}^{5}$, we observe that it only appears in the cases (a) and (c) and arguing as in Section 5.2 we can bound it as

$$
\operatorname{Err}_{i}^{5} \leq I_{1}+\underbrace{\left|\int\left\langle X_{i}^{\perp}, h\left(\overrightarrow{\mathbf{T}}_{F}(p)\right)\right\rangle d\left\|\mathbf{T}_{F}\right\|\right|}_{I_{2}},
$$

where $h\left(v_{1} \wedge v_{2}\right):=\sum_{i=1}^{2} A_{\Sigma}\left(v_{i}, v_{i}\right)$ and $I_{1}$ enjoys the same bounds as $\operatorname{Err}_{i}^{4}$. Following the argument of [8, Section 4.3] we can see that $I_{2}$ enjoys the same bounds as $\operatorname{Err}_{i}^{1}$ and $\operatorname{Err}_{i}^{2}$. 
To conclude the proof notice that, with analogous computation as in [4, Proposition 3.1],

$$
\left.\frac{d}{d \varepsilon}\right|_{\varepsilon=0} \int_{\mathcal{M}}\left|D\left(N \circ \Gamma_{\varepsilon}\right)\right|^{2}=\int_{\mathcal{M}}\left(2 \sum_{i=1}^{Q}\left\langle D N_{i}:\left(D N_{i} \cdot D_{\mathcal{M}} Y\right)\right\rangle-|D N|^{2} \operatorname{div}_{\mathcal{M}} Y\right) .
$$

However, by the conformal invariance of the Dirichlet energy, we have

$$
\int_{\mathcal{M}}\left|D\left(N \circ \Gamma_{\varepsilon}\right)\right|^{2}=\int_{\mathfrak{B}}\left|D\left(\mathcal{N} \circ \hat{\Gamma}_{\varepsilon}\right)\right|^{2},
$$

where $\hat{\Gamma}_{\varepsilon}$ is the one parameter family of diffeomorphisms generated by the vector field $\hat{Y}: \mathfrak{B} \rightarrow \mathfrak{B}$ defined by

$$
\hat{Y}(z, w):=\frac{|z|}{r} \phi\left(\frac{|z|}{r}\right) \nu
$$

Hence

$$
\left.\frac{d}{d \varepsilon}\right|_{\varepsilon=0} \int_{\mathcal{M}}\left|D\left(N \circ \Gamma_{\varepsilon}\right)\right|^{2}=\int_{\mathfrak{B}}\left(2 \sum_{i=1}^{Q}\left\langle D \mathcal{N}_{i}:\left(D \mathcal{N}_{i} \cdot D \hat{Y}\right)\right\rangle-|D \mathcal{N}|^{2} \operatorname{div} \hat{Y}\right)
$$

where the differentiation is taken with respect to the (local) flat structure of $\mathfrak{B}$.

In particular we conclude

$$
\left.\frac{d}{d \varepsilon}\right|_{\varepsilon=0} \int_{\mathcal{M}}\left|D\left(N \circ \Gamma_{\varepsilon}\right)\right|^{2}=\int_{B_{r}} \frac{|z|}{r^{2}} \phi^{\prime}\left(\frac{|z|}{r}\right)\left(2\left|D_{\nu} \mathcal{N}\right|^{2}-|D \mathcal{N}|^{2}\right) .
$$

Collecting together (6.15), (6.17) and the error estimates, and letting $\phi$ converge to the to the indicator function of $[-1,1]$ (namely letting $j \uparrow \infty$ ) we conclude the proof.

6.2. Proof of Lemma 6.2. The lemma is a very simple corollary of the estimates proven so far. (6.4) is a simple consequence of the Poincaré inequality (5.1) and of (5.13). Similarly, by Lemma 5.4, we have that $\boldsymbol{\Lambda}(r) \leq C \mathbf{D}(r)$, and therefore (6.7) follows in view of (6.4). The same arguments hold for (6.8). Next for (6.5) we can estimate as follows:

$$
\begin{aligned}
|\mathbf{L}(r)| & \leq C \boldsymbol{m}_{0}^{1 / 2} \int_{B_{r}}|\mathcal{N}||D \mathcal{N}| \leq C \boldsymbol{m}_{0}^{1 / 2}\left(\int_{0}^{r} \mathbf{H}(t) d t\right)^{\frac{1}{2}} \mathbf{D}^{\frac{1}{2}}(r) \\
& \stackrel{(5.1)}{\leq} C \boldsymbol{m}_{0}^{1 / 2}\left(C_{5.1} \int_{0}^{r} t \mathbf{D}(t) d t\right)^{\frac{1}{2}} \mathbf{D}^{\frac{1}{2}}(r) \leq C \boldsymbol{m}_{0}^{1 / 2} r \mathbf{D}(r) .
\end{aligned}
$$

Similarly

$$
\left|\mathbf{L}^{\prime}(r)\right| \leq C \boldsymbol{m}_{0}^{1 / 2} \int_{\partial B_{r}}|\mathcal{N}||D \mathcal{N}| \leq C \boldsymbol{m}_{0}^{1 / 2}\left(\mathbf{D}^{\prime}(r) \mathbf{H}(r)\right)^{\frac{1}{2}}
$$

Finally, we notice that Proposition 6.1 implies

$$
\left.\left|\frac{\mathbf{D}^{\prime}(r)}{2}-\int_{\partial B_{r}}\right| D_{\tau} \mathcal{N}\right|^{2} \mid \leq C \mathcal{E}_{I V}(r) .
$$


Therefore, using the almost minimizing property in (4.5) and the Poincaré inequality we infer that

$$
\mathbf{D}(r) \leq(1+C r)\left[\frac{r \mathbf{D}^{\prime}(r)}{2(2 a+b)}+\frac{a(a+b) \mathbf{H}(r)}{r(2 a+b)}\right]+C(a) r \mathcal{E}_{I V}(r)+\mathcal{E}_{Q M}(r)+C r^{1+\sigma} \mathbf{D}^{\prime}(r) .
$$

Absorbing the error term $r^{1+\sigma} \mathbf{D}^{\prime}(r)$ and dividing by $\left(1+C r^{\sigma}\right)$ we get

$$
\mathbf{D}(r) \leq \frac{r \mathbf{D}^{\prime}(r)}{2(2 a+b)}+\frac{a(a+b) \mathbf{H}(r)}{r(2 a+b)}+C(a) r \mathcal{E}_{I V}(r)+\mathcal{E}_{Q M}(r)+C r^{\sigma} \mathbf{D}(r),
$$

from which (6.9) follows straightforwardly by noticing that $\mathcal{E}_{Q M}(r)+r \mathbf{D}(r) \leq C r \mathcal{E}_{I V}(r)$.

6.3. Proof of Corollary 6.3. Recall first thet $\eta<\gamma_{0}$. We start with $\mathcal{E}_{B P}(r)$. Notice that, using $\mathbf{H}(t) \leq C t \mathbf{D}(t)$ together with the definition of $\mathbf{F}(r)$, we have

$$
\int_{0}^{r}\left(\frac{1}{t^{\gamma} \mathbf{D}(t)}\right)^{\prime} \mathbf{F}(t) d t \leq C \frac{\mathbf{F}(r)}{r^{\gamma} \mathbf{D}(r)}+C \int_{0}^{r} \frac{1}{t^{\gamma} \mathbf{D}(t)} \frac{\mathbf{H}(t)}{t^{2-\gamma_{0}}} d t \leq C r^{\gamma_{0}-\gamma}
$$

Next, by a simple integration by parts and the fact that $\mathbf{D}(r) \leq C r^{2}$, we deduce

$$
\begin{gathered}
\int_{0}^{r} \frac{1}{t^{\gamma} \mathbf{D}(t)} \frac{d}{d t}\left\|T-\mathbf{T}_{F}\right\|\left(\mathbf{p}^{-1}\left(\mathbf{\Psi}\left(B_{t}\right)\right)\right) d t=\frac{1}{r^{\gamma} \mathbf{D}(r)}\left\|T-\mathbf{T}_{F}\right\|\left(\mathbf{p}^{-1}\left(\mathbf{\Psi}\left(B_{r}\right)\right)\right) \\
\quad+\int_{0}^{r}\left(\frac{1}{t^{\gamma} \mathbf{D}(t)}\right)^{\prime}\left\|T-\mathbf{T}_{F}\right\|\left(\mathbf{p}^{-1}\left(\mathbf{\Psi}\left(B_{t}\right)\right)\right) d t \\
\stackrel{(2.15)}{\leq} C \frac{\mathbf{D}^{1+\eta}(r)+\mathbf{F}(r)}{r^{\gamma} \mathbf{D}(r)}+\int_{0}^{r}\left(\frac{1}{t^{\gamma} \mathbf{D}(t)}\right)^{\prime}\left(\mathbf{D}(t)^{1+\eta}+\mathbf{F}(t)\right) d t \leq C r^{\eta-\gamma} .
\end{gathered}
$$

In a similar fashion we have

$$
\begin{aligned}
\int_{0}^{r} \frac{\boldsymbol{m}_{0}^{1 / 2}}{t^{\gamma} \mathbf{D}(t)} & \int_{\partial B_{t}} \frac{|\boldsymbol{\eta} \circ \mathcal{N}(z, w)|}{t^{1-\gamma_{0}}} d t \leq \frac{\boldsymbol{m}_{0}^{1 / 2}}{r^{\gamma} \mathbf{D}(r)} \int_{B_{r}} \frac{|\boldsymbol{\eta} \circ \mathcal{N}(z, w)|}{|z|^{1-\gamma_{0}}} \\
& +\int_{0}^{r}\left(\frac{1}{t^{\gamma} \mathbf{D}(t)}\right)^{\prime} \boldsymbol{m}_{0}^{1 / 2} \int_{B_{t}} \frac{|\boldsymbol{\eta} \circ \mathcal{N}(z, w)|}{|z|^{1-\gamma_{0}}} \\
& \stackrel{(2.14)}{\leq} C \frac{\mathbf{D}^{1+\eta}(r)+\mathbf{F}(r)}{r^{\gamma} \mathbf{D}(r)}+\int_{0}^{r}\left(\frac{1}{t^{\gamma} \mathbf{D}(t)}\right)^{\prime}\left(\mathbf{D}(t)^{1+\eta}+\mathbf{F}(t)\right) d t \leq C r^{\eta-\gamma}
\end{aligned}
$$

so that

$$
\int_{0}^{r} \frac{\mathcal{E}_{B P}(t)}{t^{\gamma} \mathbf{D}(t)} d t \leq C r^{\eta-\gamma}
$$

To conclude, we estimate separately the two nonnegative functions $\boldsymbol{\Sigma}_{I V}$ and $\boldsymbol{\Sigma}_{O V}$. In particular

$$
\begin{aligned}
\boldsymbol{\Sigma}_{I V}(r) & =\int_{0}^{r} \frac{\mathcal{E}_{I V}(t)}{t^{\gamma} \mathbf{D}(t)} d t \stackrel{(6.8)}{\leq} 2 C_{6.2} \int_{0}^{r}\left(t^{\gamma_{0}-\gamma-1}+t^{-\gamma} \mathbf{D}(t)^{\eta-1} \mathbf{D}^{\prime}(t)+\frac{\mathcal{E}_{B P}(t)}{t^{\gamma} \mathbf{D}(t)}\right) d t \\
& \leq C r^{\eta-\gamma}\left(1+\mathbf{D}(t)^{\eta / 2}\right) \leq C r^{\eta-\gamma}
\end{aligned}
$$


where in the second inequality we used $\mathbf{D}(t) \leq C t^{2}$. Finally

$$
\begin{aligned}
\boldsymbol{\Sigma}_{O V}(r) & =\int_{0}^{r} \frac{\mathcal{E}_{O V}(t)}{t^{1+\gamma} \mathbf{D}(t)} d t \\
\stackrel{(6.7)}{\leq} C_{6.2} & \int_{0}^{r}\left(\frac{\mathbf{D}^{\eta}(t)}{t^{1+\gamma}}+\frac{\mathbf{F}(t)}{t^{1+\gamma} \mathbf{D}(t)}+t^{-\gamma} \mathbf{D}^{\eta-1}(t) \mathbf{D}^{\prime}(t)+\frac{\mathcal{E}_{B P}(t)}{t^{\gamma} \mathbf{D}(t)}\right) d t \\
& \leq C r^{\eta-\gamma} .
\end{aligned}
$$

\section{Almost monotonicity And DeCAy of the FREQuency FunCtion}

In this section we study the asymptotic behaviour of the normal approximation $\mathcal{N}$. The first step consists in proving approximate monotonicity and decay estimates for the frequency function.

For every $r \in(0,1)$ such that $\mathbf{H}(r)>0$, we set $\overline{\mathbf{I}}(r):=\frac{r \boldsymbol{\Omega}(r)}{\mathbf{H}(r)}$ where we recall that

$$
\boldsymbol{\Omega}(r):= \begin{cases}\mathbf{D}(r) & \text { in the cases (a) and (b) of Definition 0.1; } \\ \mathbf{D}(r)+\mathbf{L}(r) & \text { in case (c). }\end{cases}
$$

Furthermore we define $\overline{\mathbf{K}}(r):=\overline{\mathbf{I}}(r)^{-1}$ whenever $\boldsymbol{\Omega}(r) \neq 0$. By (6.5) there exists $r_{0}>0$ such that

$$
\frac{1}{2} \mathbf{D}(r) \leq(1-C r) \mathbf{D}(r) \leq \mathbf{\Omega}(r) \leq(1+C r) \mathbf{D}(r) \leq 2 \mathbf{D}(r) \quad \forall r \leq r_{0} .
$$

Having fixed $r_{0}, \overline{\mathbf{K}}(r)$ is well defined whenever $\mathbf{D}(r)>0$ and hence, by the Poincaré inequality, whenever $\overline{\mathbf{I}}(r)$ is defined. Moreover, if for some $\rho \leq r_{0} \overline{\mathbf{K}}(\rho)$ is not well defined, that is $\boldsymbol{\Omega}(\rho)=0$, then obviously $\boldsymbol{\Omega}(r)=\mathbf{D}(r)=0$ for every $r \leq \rho$.

We are now ready to state the first important monotonicity estimate. From now on we assume of having fixed a $\gamma$

Theorem 7.1. There exists a constant $C_{7.1}>0$ with the following property: if $\mathbf{D}(r)>0$ for some $r \leq r_{0}$, then (setting $\gamma=0$ in (6.10) and (6.11)) the function

$$
\overline{\mathbf{K}}(r) \exp \left(-4 r-4 \boldsymbol{\Sigma}_{I V}(r)\right)-4 \mathbf{\Sigma}_{O V}(r)
$$

is monotone non-increasing on any interval $[a, b]$ where $\mathbf{D}$ is nowhere 0 . In particular, either there is $\bar{r}>0$ such that $\mathbf{D}(\bar{r})=0$ or $\overline{\mathbf{K}}$ is well-defined on $] 0, r_{0}$ [ and the limit $K_{0}:=\lim _{r \rightarrow 0} \overline{\mathbf{K}}(r)$ exists.

A fundamental consequence of Theorem 7.1 is the following dichotomy.

Corollary 7.2. There exists $\bar{r}>0$ such that

(A) either $\overline{\mathbf{K}}(r)$ is well-defined for every $r \in] 0, r_{0}[$, the limit

$$
K_{0}:=\lim _{r \downarrow 0} \overline{\mathbf{K}}(r)
$$

is positive and thus there is a constant $C$ and a radius $\bar{r}$ such that

$$
\left.C^{-1} r \mathbf{D}(r) \leq \mathbf{H}(r) \leq C r \mathbf{D}(r) \quad \forall r \in\right] 0, \bar{r}[;
$$

(B) or $T\left\llcorner\mathbf{p}^{-1}\left(\boldsymbol{\Psi}\left(B_{\bar{r}}\right)\right)=Q \llbracket \Psi\left(B_{\bar{r}}\right) \rrbracket\right.$ for some positive $\bar{r}$. 
In turn, using the above dichotomy we will show

Theorem 7.3. Assume that condition (i) in Theorem 2.8 fails. Then the frequency $\overline{\mathbf{I}}(r)$ is well-defined for every sufficiently small $r$ and its limit $I_{0}=\lim _{r \rightarrow 0} \overline{\mathbf{I}}(r)=K_{0}^{-1}$ exists and it is finite and positive. Moreover there exist constants $\lambda, C_{7.3}, H_{0}, D_{0}>0$ such that, for every $r$ sufficiently small the following holds:

$$
\left|\mathbf{I}(r)-I_{0}\right|+\left|\frac{\mathbf{H}(r)}{r^{2 I_{0}+1}}-H_{0}\right|+\left|\frac{\mathbf{D}(r)}{r^{2 I_{0}}}-D_{0}\right| \leq C_{7.3} r^{\lambda} .
$$

7.1. Proof of Theorem 7.1. In the first step we claim the monotonicity of the function $\overline{\mathbf{K}}(r) \exp \left(-\boldsymbol{\Sigma}_{I V}(r)\right)-2 \boldsymbol{\Sigma}_{O V}(r)$ on any interval contained in $[a, b]$ on which $\mathbf{D}$ is everywhere positive. Recalling that $\boldsymbol{\Omega}$ and $\mathbf{H}$ are absolutely continuous functions, we can compute the following derivative: for every $r \in[a, b]$

$$
\begin{aligned}
\overline{\mathbf{K}}^{\prime}(r) & =\left(\frac{\mathbf{H}(r)}{r}\right)^{\prime} \frac{1}{\boldsymbol{\Omega}(r)}-\frac{\mathbf{H}(r)}{r} \frac{\mathbf{\Omega}^{\prime}(r)}{\boldsymbol{\Omega}^{2}(r)} \\
& \stackrel{(5.3)}{\leq} \frac{1}{r \boldsymbol{\Omega}^{2}(r)}\left(2 \mathbf{E}(r) \boldsymbol{\Omega}(r)-\mathbf{D}^{\prime}(r) \mathbf{H}(r)+\left|\mathbf{L}^{\prime}(r)\right| \mathbf{H}(r)\right)
\end{aligned}
$$

Then, either $\overline{\mathbf{K}}^{\prime} \leq 0$, or the RHS of the inequality above is positive, that is

$$
\mathbf{D}^{\prime}(r) \mathbf{H}(r) \leq 2 \mathbf{E}(r) \boldsymbol{\Omega}(r)+\left|\mathbf{L}^{\prime}(r)\right| \mathbf{H}(r) \stackrel{(6.6)}{\leq} 2 \mathbf{E}(r) \boldsymbol{\Omega}(r)+r \mathbf{D}^{\prime}(r) \mathbf{H}(r)+\frac{\mathbf{H}^{2}(r)}{r} .
$$

In turn, using $\mathbf{H}(r) \leq C r \mathbf{D}(r) \leq C r \boldsymbol{\Omega}(r)$, the latter inequality implies

$$
\mathbf{D}^{\prime}(r) \mathbf{H}(r) \leq C \mathbf{E}(r) \boldsymbol{\Omega}(r)+C r \boldsymbol{\Omega}^{2}(r) .
$$

From this we deduce

$$
\mathbf{E}^{2}(r) \leq \mathbf{H}(r) \mathbf{G}(r) \leq \mathbf{H}(r) \mathbf{D}^{\prime}(r) \leq C \boldsymbol{\Omega}^{2}(r)+\frac{\mathbf{E}^{2}(r)}{2}
$$

which implies that $\mathbf{E}(r) \leq C \Omega(r)$ and so, by (6.6),

$$
\left|\mathbf{L}^{\prime}(r)\right| \leq C \boldsymbol{m}_{0}^{1 / 2}\left(\mathbf{D}^{\prime}(r) \mathbf{H}(r)\right)^{1 / 2} \leq C \boldsymbol{m}_{0}^{1 / 2} \boldsymbol{\Omega}(r) .
$$

Next using again the Cauchy-Schwarz inequality and (5.4), we have

$$
\begin{aligned}
\boldsymbol{\Omega}(r) \mathbf{E}(r) & \leq \boldsymbol{\Omega}(r) \mathbf{H}(r)^{1 / 2} \mathbf{G}(r)^{1 / 2} \leq \frac{\boldsymbol{\Omega}(r)^{2}}{2}+\frac{\mathbf{H}(r) \mathbf{G}(r)}{2} \\
& \leq \frac{\boldsymbol{\Omega}(r) \mathbf{E}(r)}{2}+\frac{\boldsymbol{\Omega}(r) \mathcal{E}_{O V}(r)}{2}+\frac{\mathbf{H}(r) \mathbf{G}(r)}{2}
\end{aligned}
$$

which implies

$$
\boldsymbol{\Omega}(r) \mathbf{E}(r) \leq \mathbf{H}(r) \mathbf{G}(r)+\mathbf{\Omega}(r) \mathcal{E}_{O V}(r) .
$$


Collecting all these estimates together and using (6.2), we conclude that, if $\overline{\mathbf{K}}^{\prime}(r) \geq 0$, then

$$
\begin{gathered}
\overline{\mathbf{K}}^{\prime}(r) \stackrel{(7.6) \&(7.9)}{\leq} \frac{1}{r \boldsymbol{\Omega}^{2}(r)}\left(2 \mathbf{H}(r) \mathbf{G}(r)-\mathbf{D}^{\prime}(r) \mathbf{H}(r)+\left|\mathbf{L}^{\prime}(r)\right| \mathbf{H}(r)+2 \boldsymbol{\Omega}(r) \mathcal{E}_{O V}(r)\right) \\
\stackrel{(6.2) \&(7.8)}{\leq} \frac{1}{r \boldsymbol{\Omega}^{2}(r)}\left(2 \mathbf{H}(r) \mathbf{G}(r)-2 \mathbf{H}(r) \mathbf{G}(r)+\mathbf{\Omega}(r) \mathbf{H}(r)+\mathbf{H}(r) \mathcal{E}_{I V}(r)+2 \boldsymbol{\Omega}(r) \mathcal{E}_{O V}(r)\right) \\
\leq 2 \frac{\mathcal{E}_{O V}(r)}{r \boldsymbol{\Omega}(r)}+\overline{\mathbf{K}}(r)\left(1+\frac{\mathcal{E}_{I V}(r)}{\Omega(r)}\right) \leq 4 \frac{\mathcal{E}_{O V}(r)}{r \mathbf{D}(r)}+4 \overline{\mathbf{K}}(r)\left(1+\frac{\mathcal{E}_{I V}(r)}{\mathbf{D}(r)}\right) .
\end{gathered}
$$

On the other hand the final inequality

$$
\overline{\mathbf{K}}^{\prime}(r) \leq 4 \frac{\mathcal{E}_{O V}(r)}{r \mathbf{D}(r)}+4 \overline{\mathbf{K}}(r)\left(1+\frac{\mathcal{E}_{I V}(r)}{\mathbf{D}(r)}\right)
$$

is certainly correct when $\overline{\mathbf{K}}^{\prime}(r) \leq 0$, because the right hand side is positive. The monotonicity of the function in (7.2) is then obvious.

Next, as already observed, either $\mathbf{D}$ is always positive, or it vanishes on some interval ]0, $\bar{r}$. If $\mathbf{D}$ is always positive, then $\overline{\mathbf{K}}$ is well defined on $] 0, r_{0}$ [ and the existence of the limit $K_{0}:=\lim _{r \downarrow 0} \overline{\mathbf{K}}(r)$ is a direct consequence of (7.2) and Corollary 6.3.

7.2. Proof of Corollary 7.2. First of all observe that, if $\mathbf{D}(\bar{r})$ vanishes, then $\mathcal{N} \equiv Q \llbracket 0 \rrbracket$ on $B_{\bar{r}}$. In particular by $(2.15)$ we conclude that we are in the alternative (B). We can thus assume, without loss of generality, that $\mathbf{D}$ is positive on $] 0, r_{0}\left[\right.$. Assuming that $K_{0}$ vanishes we will then reach a contradiction.

Under the assumption $K_{0}=0$, consider the monotonicity of $\overline{\mathbf{K}}(r) \exp \left(-4 \boldsymbol{\Sigma}_{I V}(r)\right)-$ $4 \boldsymbol{\Sigma}_{O V}(r)$ between two radii $0<s<r$ and let $s \rightarrow 0$ to get

$$
\overline{\mathbf{K}}(r) \leq 4 e^{4 r+4 \boldsymbol{\Sigma}_{I V}(r)} \boldsymbol{\Sigma}_{O V}(r) \leq C \boldsymbol{\Sigma}_{O V}(r),
$$

where the last inequality holds for $r$ sufficiently small, since $\boldsymbol{\Sigma}_{I V}(r) \leq C r^{\eta}$ (recall that we have set $\gamma=0)$. Next observe that, since the function $\boldsymbol{\Sigma}_{O V}(r)$ is non-decreasing,

$$
\frac{\mathbf{F}(r)}{\mathbf{D}(r)} \leq \frac{1}{\mathbf{D}(r)} \int_{0}^{r} \frac{\mathbf{H}(s)}{s^{2-\gamma_{0}}} \frac{\mathbf{D}(s)}{\mathbf{D}(s)} d s \leq C \int_{0}^{r} \frac{\overline{\mathbf{K}}(s)}{s^{1-\gamma_{0}}} d s \leq C r^{\gamma_{0}} \boldsymbol{\Sigma}_{O V}(r) .
$$

Moreover, integrating by parts:

$$
\begin{aligned}
& \int_{0}^{r} \frac{1}{\mathbf{D}(s)} \frac{d}{d s}\left\|T-\mathbf{T}_{F}\right\|\left(\mathbf{p}^{-1}\left(\mathbf{\Psi}\left(B_{s}\right)\right)\right) d s \\
\stackrel{(2.15)}{\leq} & C \frac{\mathbf{D}^{1+\eta}(r)+\mathbf{F}(r)}{\mathbf{D}(r)}+C \int_{0}^{r}\left(\frac{1}{\mathbf{D}(s)}\right)^{\prime}\left(\mathbf{D}^{1+\eta}(s)+\mathbf{F}(s)\right) d s \\
\leq & C \mathbf{D}^{\eta}(r)+C r^{\gamma_{0}} \boldsymbol{\Sigma}_{O V}(r)+C \frac{\mathbf{F}(r)}{\mathbf{D}(r)}+C \int_{0}^{r} \frac{\mathbf{F}^{\prime}(s)}{\mathbf{D}(s)} d s \\
\leq & C \mathbf{D}^{\eta}(r)+C r^{\gamma_{0}} \boldsymbol{\Sigma}_{O V}(r)+C \int_{0}^{r} \frac{\overline{\mathbf{K}}(s)}{s^{1-\gamma_{0}}} d s \leq C \mathbf{D}^{\eta}(r)+C r^{\gamma_{0}} \boldsymbol{\Sigma}_{O V}(r),
\end{aligned}
$$

where we have used repeatedly (7.1). 
Using the latter in the formula for $\mathcal{E}_{O V}$ we also conclude

$$
\begin{aligned}
& \boldsymbol{\Sigma}_{O V}(r) \\
\leq & C \int_{0}^{r} \frac{1}{s \mathbf{D}(s)}\left(\mathbf{D}(s)^{1+\eta}+s \mathbf{D}^{\eta}(s) \mathbf{D}^{\prime}(s)+\mathbf{F}(s)+s \frac{d}{d s}\left\|T-\mathbf{T}_{F}\right\|\left(\mathbf{p}^{-1}\left(\mathbf{\Psi}\left(B_{s}\right)\right)\right)\right) d s \\
\leq & C r^{\eta} \mathbf{D}(r)^{\eta / 2}+C r^{\gamma_{0}} \boldsymbol{\Sigma}_{O V}(r) .
\end{aligned}
$$

Hence, for $r$ sufficiently small,

$$
\overline{\mathbf{K}}(r) \leq C \boldsymbol{\Sigma}_{O V}(r) \leq C \mathbf{D}(r)^{\eta / 2} .
$$

In particular this implies that

$$
\mathbf{H}(r) \leq C r \mathbf{D}(r)^{1+\eta / 2} .
$$

Combining this with (5.4) and the Cauchy-Schwarz inequality, we deduce

$$
\begin{aligned}
\frac{1}{2} \mathbf{D}(r) & \leq \mathbf{\Omega}(r) \leq \frac{\mathbf{E}(r)}{r}+\mathcal{E}_{O V}(r) \leq\left(\frac{\mathbf{H}(r)}{r \mathbf{D}(r)^{\eta / 4}}\right)^{1 / 2}\left(r \mathbf{D}^{\prime}(r) \mathbf{D}(r)^{\eta / 4}\right)^{1 / 2}+\mathcal{E}_{O V}(r) \\
& \stackrel{(7.14)}{\leq} C \mathbf{D}(r)^{1+\eta / 4}+C r \mathbf{D}(r)^{\eta / 4} \mathbf{D}^{\prime}(r)+\mathcal{E}_{O V}(r) .
\end{aligned}
$$

Dividing the expression above by $r \mathbf{D}(r)$, integrating between two radii $0<s<r$ and using the bound $\mathbf{D}(r) \leq C r^{2}$ we obtain

$$
\log \left(\frac{r}{s}\right) \leq C \int_{s}^{r}\left(\frac{\mathbf{D}(\rho)^{\eta / 4}}{\rho}+\mathbf{D}(\rho)^{\eta / 4-1} \mathbf{D}^{\prime}(\rho)+\frac{\mathcal{E}_{O V}(\rho)}{\rho \mathbf{D}(\rho)}\right) d \rho \leq C r^{\eta / 2} .
$$

Sending $s \rightarrow 0$ we get a contradiction.

7.3. Proof of Theorem 7.3. Clearly, if (i) in Theorem 2.8 does not hold, then $\mathbf{D}$ is always positive and we are in alternative (A) of Corollary 7.2. Thus $K_{0}$ is positive and the first statement is obvious.

Let $\mathbf{K}(r):=\mathbf{I}(r)^{-1}$ and observe that by (7.1) we have

$$
(1-C r) \mathbf{I}(r) \leq \overline{\mathbf{I}}(r) \leq(1+C r) \mathbf{I}(r), \quad \forall 0 \leq r \leq r_{0},
$$

which implies

$$
(1-C r) \overline{\mathbf{K}}(r) \leq \mathbf{K}(r) \leq(1+C r) \overline{\mathbf{K}}(r) \quad \forall 0 \leq r \leq r_{0},
$$

so that in particular $\mathbf{K}(r) \leq C \overline{\mathbf{K}}(r)<\infty$ for every $0<r<r_{0}$ and $\mathbf{K}(r) \rightarrow K_{0}$ as $r \rightarrow 0$. Using the monotonicity formula of Theorem 7.1 together with Corollary 6.3 we have

$$
\overline{\mathbf{K}}(r)-K_{0} \leq K_{0}\left(\exp \left(4 r+4 \boldsymbol{\Sigma}_{O V}(r)\right)-1\right)+4 \Sigma_{I V}(r) \exp \left(4 r+4 \Sigma_{O V}(r)\right) \leq C r^{\eta} .
$$

Therefore

$$
\mathbf{K}(r)-K_{0} \leq C r^{\eta}+C \mathbf{K}(r) r \leq C r^{\eta}
$$

To control $\mathbf{K}(r)-K_{0}$ from below we apply (6.9) with $a=I_{0}=\frac{1}{K_{0}}$ and $b=\lambda \leq$ $\min \left\{\eta / 2, b_{0}\left(I_{0}\right)\right\}$ to infer, after dividing by $r \mathbf{D}(r)$, that

$$
-\frac{\mathbf{D}^{\prime}(r)}{\mathbf{D}(r)} \leq \frac{2}{r}\left(I_{0}\left(I_{0}+\lambda\right) \mathbf{K}(r)-\left(2 I_{0}+\lambda\right)\right) .
$$


Multiplying this expression by $\mathbf{K}(r)>0$ and adding $2 / r$, we get

$$
\begin{aligned}
\frac{2}{r}-\frac{\mathbf{D}^{\prime}(r)}{\mathbf{D}(r)} \mathbf{K}(r) & \leq \frac{2}{r}\left[1+I_{0}\left(I_{0}+\lambda\right) \mathbf{K}^{2}(r)-\left(2 I_{0}+\lambda\right) \mathbf{K}(r)\right]+\frac{C \mathcal{E}_{I V}(r)}{\mathbf{D}(r)} \\
& \leq \frac{2}{r} I_{0}\left(\mathbf{K}(r)-\frac{1}{I_{0}}\right)\left(\left(I_{0}+\lambda\right) \mathbf{K}(r)-1\right)+\frac{C \mathcal{E}_{I V}(r)}{\mathbf{D}(r)}
\end{aligned}
$$

Since $\left(I_{0}+\lambda\right) \mathbf{K}(r)$ converges to $1+\lambda K_{0}$, we easily deduce that for $r$ small enough $\left(I_{0}+\right.$ $\lambda) \mathbf{K}(r)-1 \geq \frac{\lambda}{2} K_{0}$. Using this together with (7.16), we deduce from (7.17) that

$$
\frac{2}{r}-\frac{\mathbf{D}^{\prime}(r)}{\mathbf{D}(r)} \leq \frac{\lambda}{r}\left(\mathbf{K}(r)-\frac{1}{I_{0}}\right)+\frac{C \mathcal{E}_{I V}(r)}{\mathbf{D}(r)}+C \frac{r^{\eta}}{r}
$$

We next compute

$$
\begin{aligned}
\mathbf{K}^{\prime}(r) & =\left(\frac{\mathbf{H}(r)}{r}\right)^{\prime} \frac{1}{\mathbf{D}(r)}-\frac{\mathbf{H}(r)}{r \mathbf{D}(r)} \frac{\mathbf{D}^{\prime}(r)}{\mathbf{D}(r)} \stackrel{(5.3) \&(7.1)}{\leq} \frac{2 \mathbf{E}(r)}{r \mathbf{D}(r)}-\frac{\mathbf{D}^{\prime}(r)}{\mathbf{D}(r)} \mathbf{K}(r) \\
& \stackrel{(5.4) \&(7.1)}{\leq} \frac{2}{r}+C-\frac{\mathbf{D}^{\prime}(r)}{\mathbf{D}(r)} \mathbf{K}(r)+C \frac{\mathcal{E}_{O V}(r)}{r \mathbf{D}(r)} \\
& \stackrel{(7.18)}{\leq} \frac{\lambda}{r}\left(\mathbf{K}(r)-\frac{1}{I_{0}}\right)+\frac{C \mathcal{E}_{I V}(r)}{\mathbf{D}(r)}+C \frac{\mathcal{E}_{O V}(r)}{r \mathbf{D}(r)}+C \frac{r^{\eta}}{r}
\end{aligned}
$$

Recalling that $\mathbf{K}(r) \leq C$, we deduce

$$
\frac{d}{d r}\left[\frac{\mathbf{K}(r)-K_{0}}{r^{\lambda}}\right] \leq C \frac{\mathcal{E}_{O V}(r)}{r^{1+\lambda} \mathbf{D}(r)}+C \frac{\mathcal{E}_{I V}(r)}{r^{\lambda} \mathbf{D}(r)}+C \frac{1}{r^{1+\lambda-\eta}} .
$$

Integrating (7.20) on the interval ] $s, r$ [ and using (6.13), we get

$$
\mathbf{K}(r)-K_{0} \leq \frac{r^{\lambda}}{s^{\lambda}}\left(\mathbf{K}(s)-K_{0}\right)+C r^{\eta-\lambda}
$$

that is $\mathbf{K}(s)-K_{0} \geq-C s^{\lambda}$. The inequality $\left|\mathbf{K}(r)-K_{0}\right| \leq C r^{\lambda}$ easily implies $\left|\mathbf{I}(r)-I_{0}\right| \leq$ $C r^{\lambda}$.

For what concerns the other inequalities we compute

$$
\begin{aligned}
{\left[\log \left(\frac{\mathbf{H}(r)}{r^{2 I_{0}+1}}\right)\right]^{\prime} } & =\frac{\mathbf{H}^{\prime}(r)}{\mathbf{H}(r)}-\frac{2 I_{0}+1}{r}=\frac{2 \mathbf{E}(r)}{r \mathbf{H}(r)}-\frac{2 I_{0}}{r} \leq \frac{2 \mathbf{D}(r)}{\mathbf{H}(r)}-\frac{2 I_{0}}{r}+C \frac{\mathcal{E}_{O V}(r)}{\mathbf{H}(r)} \\
& =\frac{2}{r}\left(\mathbf{I}(r)-I_{0}\right)+C \frac{\mathcal{E}_{O V}(r)}{\mathbf{H}(r)}
\end{aligned}
$$

and similarly

$$
\left[\log \left(\frac{\mathbf{H}(r)}{r^{2 I_{0}+1}}\right)\right]^{\prime} \geq \frac{2}{r}\left(\mathbf{I}(r)-I_{0}\right)-C \frac{\mathcal{E}_{O V}(r)}{\mathbf{H}(r)} .
$$

Using that $\left|\mathbf{I}(r)-I_{0}\right| \leq C r^{\lambda}$, for $r$ small enough we have the bound $r \mathbf{D}(r) \leq 2 I_{0} \mathbf{H}(r)$. Hence we can use (6.13) in the integrals of (7.21) and (7.22) to deduce the existence of the 
limit

$$
H_{0}:=\lim _{s \downarrow 0} \frac{\mathbf{H}(s)}{s^{2 I_{0}+1}}, \quad \text { with } \quad\left|\frac{\mathbf{H}(r)}{r^{2 I_{0}+1}}-H_{0}\right| \leq C r^{\lambda} .
$$

Moreover, from (7.21) we also infer that for $r$ sufficiently small

$$
H_{0} \geq \frac{\mathbf{H}(r)}{r^{2 I_{0}+1}} e^{-C r^{\lambda}}>0 .
$$

Finally the last assertion follows simply setting $D_{0}:=I_{0} \cdot H_{0}$ and from

$$
\begin{aligned}
\left|\frac{\mathbf{D}(r)}{r^{2 I_{0}}}-D_{0}\right| & =\left|\mathbf{I}(r) \frac{\mathbf{H}(r)}{r^{2 I_{0}+1}}-I_{0} H_{0}\right| \\
& \leq\left|\mathbf{I}(r)-I_{0}\right| \frac{\mathbf{H}(r)}{r^{2 I_{0}+1}}+I_{0}\left|\frac{\mathbf{H}(r)}{r^{2 I_{0}+1}}-H_{0}\right| \leq C r^{\lambda} .
\end{aligned}
$$

\section{BLOW-UP AND PROOF OF ThEOREM 2.8}

As a consequence of the decay estimate in Theorem 7.3 we can show that suitable rescaling of the normal approximation $N$ converge to a unique limiting profile. To this aim we consider for every $r \in(0,1)$ the functions $f_{r}: \partial B_{1} \rightarrow \mathcal{A}_{Q_{1}}\left(\mathbb{R}^{2+n}\right)$ given by

$$
f_{r}(z, w):=\frac{\mathcal{N}\left(i_{r}(z, w)\right)}{r^{I_{0}}}
$$

where we recall that $i_{r}(z, w)=\left(r z, r^{1 / \bar{Q}} w\right)$. We recall also that $T_{0} \mathcal{M}=\mathbb{R}^{2} \times\{0\}$, and $T_{0} \Sigma=\mathbb{R}^{2} \times \mathbb{R}^{\bar{n}} \times\{0\}$. In the following, with a slight abuse of notation, we write $\mathbb{R}^{\bar{n}}$ for the subspace $\{0\} \times \mathbb{R}^{\bar{n}} \times\{0\}$.

The final step in the proof of Theorem 2.8 is then the following proposition.

Proposition 8.1. Assume alternative (i) in Theorem 2.8 fails and let $I_{0}$ and $\lambda$ be the positive numbers of Theorem 7.3. Then $I_{0}>1$ and there exists a function $f_{0}: \partial B_{1} \rightarrow$ $\mathcal{A}_{Q}\left(\mathbb{R}^{\bar{n}}\right)$ such that

(i) $\boldsymbol{\eta} \circ f_{0}=0$ and $f_{0} \not \equiv Q_{1} \llbracket 0 \rrbracket$;

(ii) for every $r$ sufficiently small

$$
\mathcal{G}\left(f_{r}(z, w), f_{0}(z, w)\right) \leq C r^{\lambda / 16} \quad \forall(z, w) \in \partial B_{1}
$$

(iii) the $I_{0}$-homogeneous extension $g(z, w):=|z|^{I_{0}} f_{0}\left(\frac{z}{|z|}, \frac{w}{|w|}\right)$ is nontrivial and Dirminimizing.

In particular, by (iii) $\operatorname{Im}(g) \backslash\{0\} \subset \mathbb{R}^{2+n}$ is a real analytic submanifold.

Theorem 2.8 follows immediately from Proposition 8.1 and Theorem 7.3.

Proof of Theorem 2.8. Since we have identitified $\mathbb{R}^{\bar{n}}$ with $\{0\} \times \mathbb{R}^{\bar{n}} \times\{0\}$, it is obvious that the map $g$ has all the properties claimed in (ii), namely it is Dir-minimizing, $\boldsymbol{\eta} \circ g \equiv 0$ and it is nontrivial. (2.16) is a corollary of (8.1) provided $a_{0} \leq \frac{\lambda}{16}$. Next note that (2.18) has 
been shown in Theorem 5.1. As for (2.17) observe that, if $4 \rho \leq r<1$, then, by Theorem 7.3 ,

$D_{0}(r-2 \rho)^{2 I_{0}}-C(r-2 \rho)^{2 I_{0}+\lambda} \leq \mathbf{D}(r-2 \rho) \leq \mathbf{D}(r+2 \rho) \leq D_{0}(r+2 \rho)^{2 I_{0}}+C(r+2 \rho)^{2 I_{0}+\lambda}$.

Since $2 I_{0}>2,(2.17)$ follows easily from

$$
\int_{B_{r+2 \rho} \backslash B_{r-2 \rho}}|D \mathcal{N}|^{2}=\mathbf{D}(r+2 \rho)-\mathbf{D}(r-2 \rho),
$$

provided $a_{0} \leq \lambda$.

The rest of this final section of the note is devoted to the proof Proposition 8.1, which is split in several steps. Before starting with it, let us however observe that the conclusion $I_{0}>1$ is an obvious consequence of the decay estimates of Theorem 7.3 and the fact that $\mathbf{D}(r) \leq C r^{2+2 \gamma_{0}}$.

8.1. Step 1: uniqueness of the limit $f_{0}$. For $r$ sufficienly small and $s \in\left[\frac{r}{2}, r\right]$, we start estimating the following quantity:

$$
\int_{\partial B_{1}} \mathcal{G}\left(f_{r}, f_{s}\right)^{2} \leq(r-s) \int_{\partial B_{1}} \int_{s}^{r}\left|\frac{d}{d t} f_{t}(z, w)\right|^{2} d t .
$$

Using the differentiability properties of Lipschitz multiple valued functions and the 1dimensional theory in [4, Section 1.1.2] (note that $t \mapsto \mathcal{N}\left(i_{t}(z, w)\right)$ is a Lipschitz map), we easily infer that

$$
\begin{aligned}
\left|\frac{d}{d t} f_{t}(z, w)\right|^{2} & =\sum_{j=1}^{Q}\left|\frac{D \mathcal{N}_{j}\left(i_{t}(z, w)\right) \cdot z}{t^{I_{0}}}-I_{0} \frac{\mathcal{N}_{j}\left(i_{t}(z, w)\right)}{t^{I_{0}+1}}\right|^{2} \\
& =\frac{|z|^{2}\left|\partial_{\hat{r}} \mathcal{N}\right|^{2}\left(i_{t}(z, w)\right)}{t^{2 I_{0}}}-2 I_{0} \frac{|z|}{t^{2 I_{0}+1}} \sum_{j=1}^{Q}\left\langle\partial_{\hat{r}} \mathcal{N}_{j}, \mathcal{N}_{j}\right\rangle\left(i_{t}(z, w)\right)+\frac{|\mathcal{N}|^{2}\left(i_{t}(z, w)\right)}{t^{2 I_{0}+2}} .
\end{aligned}
$$

Therefore, by the change of variable $\left(z^{\prime}, w^{\prime}\right)=i_{t}(z, w)$ in (8.2) we infer that

$$
\begin{array}{rl}
\int_{\partial B_{1}} & \mathcal{G} \\
& \left(f_{r}, f_{s}\right)^{2} \leq \frac{r}{2} \int_{r / 2}^{r}\left(\frac{\mathbf{G}(t)}{t^{2 I_{0}+1}}-2 I_{0} \frac{\mathbf{E}(t)}{t^{2 I_{0}+2}}+I_{0}^{2} \frac{\mathbf{H}(t)}{t^{2 I_{0}+3}}\right) d t \\
& \frac{r}{2} \int_{r / 2}^{r}\left(\frac{\mathbf{D}^{\prime}(t)}{2 t^{2 I_{0}+1}}-2 I_{0} \frac{\mathbf{D}(t)}{t^{2 I_{0}+2}}+I_{0}^{2} \frac{\mathbf{H}(t)}{t^{2 I_{0}+3}}+C \frac{\mathcal{E}_{I V}(t)}{t^{2 I_{0}+1}}+C \frac{\mathcal{E}_{O V}(t)}{t^{2 I_{0}+2}}\right) d t \\
& =\frac{r}{2} \int_{r / 2}^{r}\left[\frac{1}{2 t}\left(\frac{\mathbf{D}(t)}{t^{2 I_{0}}}\right)^{\prime}+I_{0} \frac{\mathbf{H}(t)}{t^{2 I_{0}+3}}\left(I_{0}-\mathbf{I}(t)\right)+C \frac{\mathcal{E}_{I V}(t)}{t^{2 I_{0}+1}}+C \frac{\mathcal{E}_{O V}(t)}{t^{2 I_{0}+2}}\right] d t
\end{array}
$$

Using Theorem 7.3, we can then conclude that

$$
\begin{aligned}
\int_{\partial B_{1}} \mathcal{G}\left(f_{r}, f_{s}\right)^{2} & \leq C\left|\frac{\mathbf{D}(r)}{r^{2 I_{0}}}-\frac{\mathbf{D}\left(\frac{r}{2}\right)}{\left(\frac{r}{2}\right)^{2 I_{0}}}\right|+C \int_{r / 2}^{r}\left[\frac{\left|I_{0}-\mathbf{I}(t)\right|}{t}+C \frac{\mathcal{E}_{I V}(t)}{\mathbf{D}(t)}+C \frac{\mathcal{E}_{O V}(t)}{t \mathbf{D}(t)}\right] d t \\
& \leq C r^{\lambda}
\end{aligned}
$$


By an elementary dyadic argument analogous to that of [4, Theorem 5.3], we then infer the existence of $f_{0}: \partial B_{1} \rightarrow \mathcal{A}_{Q}\left(\mathbb{R}^{2+n}\right)$ such that, for $r$ sufficiently small,

$$
\left\|\mathcal{G}\left(f_{r}, f_{0}\right)\right\|_{L^{2}\left(\partial B_{1}\right)}^{2} \leq C r^{\lambda} .
$$

8.2. Step 2: uniform convergence. Set next $h(z, w):=\mathcal{G}\left(\frac{\mathfrak{N}(z, w)}{|z|^{I_{0}}}, \frac{\mathfrak{N}\left(i_{1 / 2}(z, w)\right)}{|z / 2|^{I_{0}}}\right)$. It follows from (8.3) that for $r$ sufficiently small

$$
\int_{B_{r}} h^{2} \leq \int_{0}^{r} \int_{\partial B_{1}} \mathcal{G}\left(f_{t}, f_{t / 2}\right)^{2} t d t \stackrel{(8.3)}{\leq} C r^{2+\lambda},
$$

and from (2.12) and (2.13)

$$
\operatorname{Lip}\left(\left.h\right|_{B_{1} \backslash B_{s}}\right) \leq C s^{-I_{0}} .
$$

Moreover, for every $\rho<|z| / 4$ we claim the estimate

$$
\int_{B_{\rho}(z, w)}|D h|^{2} \leq C \rho+C|z|^{\lambda} .
$$

Indeed $|D h| \leq C\left|D\left(\frac{\mathcal{N}}{|z|^{I_{0}}}\right)\right|$ and by Theorem 7.3

$$
\begin{aligned}
\int_{B_{\rho}(z, w)}\left|D\left(\frac{\mathcal{N}}{|z|^{I_{0}}}\right)\right|^{2} & \leq 2 \int_{|z|-\rho}^{|z|+\rho} \int_{\partial B_{t}}\left(\frac{|D \mathcal{N}|^{2}}{t^{2 I_{0}}}+I_{0}^{2} \frac{|\mathcal{N}|^{2}}{t^{2 I_{0}+2}}\right) d t \\
& \leq 2 \int_{|z|-\rho}^{|z|+\rho}\left(\left(\frac{\mathbf{D}(t)}{t^{2 I_{0}}}\right)^{\prime}+2 I_{0} \frac{\mathbf{D}(t)}{t^{2 I_{0}+1}}+I_{0}^{2} \frac{\mathbf{H}(t)}{t^{2 I_{0}+2}}\right) d t \\
& \leq C(|z|+\rho)^{\lambda}+C \log \left(\frac{|z|+\rho}{|z|-\rho}\right) \leq C|z|^{\lambda}+C \frac{\rho}{|z|}
\end{aligned}
$$

In particular, applying (8.5), (8.6) and (8.7) with $\rho=|z|^{1+\lambda / 4}$, we infer that for every point $p=(z, w) \in \mathfrak{B}_{\bar{Q}}$ with $|z|$ sufficiently small

$$
\begin{aligned}
h(p) & \leq\left|h(p)-f_{B_{\frac{|z|^{1+\lambda / 4}}{2^{k}}}} h\right|+\sum_{i=0}^{k-1}\left|f_{B_{\frac{|z|^{1+\lambda / 4}}{2^{i}}}} h-f_{B_{\frac{\left.|z|\right|^{1+\lambda / 4}}{2^{i+1}}}(p)} h\right|+f_{B_{|z|^{1+\lambda / 4}}(p)} h \\
& \leq \operatorname{Lip}\left(\left.h\right|_{B_{1}(p) \backslash B_{|z| / 2}(p)} \frac{|z|^{1+\lambda / 4}}{2^{k}}+C \sum_{i=0}^{k-1} \frac{|z|^{1+\lambda / 4}}{2^{i}} f_{B_{\frac{|z|^{1+\lambda / 4}}{2^{i}}}}|D h|+f_{B_{|z|^{1+\lambda / 4}}(p)} h\right. \\
& \stackrel{(8.6)}{\leq} C|z|^{1+\lambda / 4}+C \sum_{i=0}^{k-1}\left(\int_{B_{|z|^{1+\lambda / 4}}}|D h|^{2}\right)^{\frac{1}{2}}+\frac{C}{|z|^{1+\lambda / 4}}\left(\int_{B_{2|z|}}|h|^{2}\right)^{\frac{1}{2}},
\end{aligned}
$$

where we have used the standard Poincaré inequality

$$
\left|f_{B_{r}} f-f_{B_{\frac{r}{2}}} f\right| \leq C r f_{B_{r}}|D f| \quad f \in W^{1,2} .
$$


Now choose $k \in \mathbb{N}$ such that $\frac{|z|^{1+\lambda / 4}}{2^{k}}<|z|^{1+\lambda / 4+I_{0}} \leq \frac{|z|^{1+\lambda / 4}}{2^{k-1}}$ (in particular $k \leq|\log | z||$ ) and use (8.5) together with (8.7) to bound

$$
h(z, w) \leq C|z|^{1+\lambda / 4}+C|\log | z|||z|^{\lambda / 8}+C|z|^{\lambda / 4} \leq C|z|^{\lambda / 16},
$$

This gives that, for a sufficiently small $r$,

$$
\max _{\partial B_{1}} \mathcal{G}\left(f_{r}, f_{r / 2}\right) \leq C r^{\lambda / 16}
$$

Thus

$$
\max _{\partial B_{1}} \mathcal{G}\left(f_{r}, f_{0}\right) \leq \sum_{k=0}^{\infty} \mathcal{G}\left(f_{r 2^{-k}}, f_{r 2^{-k-1}}\right) \leq C r^{\lambda / 16}
$$

8.3. Step 3: nontriviality of the limit and other properties. To show that $f_{0} \neq$ $Q \llbracket 0 \rrbracket$ it is enough to observe that, by Theorem 7.3,

$$
\int_{\partial B_{1}}\left|f_{0}\right|^{2}=\lim _{r \rightarrow 0} \int_{\partial B_{1}}\left|f_{r}\right|^{2}=\lim _{r \rightarrow 0} \frac{\mathbf{H}(r)}{r^{2 I_{0}+1}}=H_{0}>0 .
$$

In order to show that $\boldsymbol{\eta} \circ f_{0} \equiv 0$, we notice that by a simple slicing argument combined with (2.14) there exists a sequence of radii $r_{k} \in\left[2^{-k-1}, 2^{-k}\right]$ such that

$$
\begin{aligned}
\int_{\partial B_{r_{k}}}|\boldsymbol{\eta} \circ \mathcal{N}| & \leq 2^{k+1} \int_{B_{2-k} \backslash B_{2^{-k-1}}}|\boldsymbol{\eta} \circ \mathcal{N}| \leq C r_{k}^{\gamma_{0}} \int_{B_{2^{-k}}}|z|^{\gamma_{0}-1}|\boldsymbol{\eta} \circ \mathcal{N}| \\
& \leq C r_{k}^{\gamma_{0}+2 \eta} \mathbf{D}\left(2 r_{k}\right) \leq C r_{k}^{\gamma_{0}+2 \eta+2 I_{0}},
\end{aligned}
$$

from which

$$
\begin{aligned}
\int_{\partial B_{1}}\left|\boldsymbol{\eta} \circ f_{0}\right| & =\lim _{r_{k} \rightarrow 0} \int_{\partial B_{1}}\left|\boldsymbol{\eta} \circ f_{r_{k}}\right|=\lim _{r_{k} \rightarrow 0} r_{k}^{-I_{0}-1} \int_{\partial B_{r}}|\boldsymbol{\eta} \circ \mathcal{N}| \\
& \leq C \lim _{r_{k} \rightarrow 0} r_{k}^{\gamma_{0}+2 \eta+I_{0}-1}=0 .
\end{aligned}
$$

Next we show that $f_{0}$ takes values in $\mathbb{R}^{\bar{n}}$. We start by showing that $f_{0}$ must take values in $T_{0} \Sigma=\mathbb{R}^{2+\bar{n}} \times\{0\}$. Indeed, if we set $f_{r}(z, w):=\overline{\mathcal{N}}\left(i_{r}(z, w)\right)$, using (A.3) and $|\mathcal{N}|\left(i_{r}(z, w)\right) \leq$ $C r^{1+\gamma_{0} / 2}$ we conclude

$$
\int_{\partial B_{1}} \mathcal{G}\left(f_{r}, \bar{f}_{r}\right)^{2} \leq \frac{C r^{2}}{r^{2 I_{0}+1}} \int_{\partial B_{r}}|\mathcal{N}|^{2} \leq C r^{2},
$$

which implies that $f_{0}(z, w) \in \mathcal{A}_{Q}\left(T_{0} \Sigma\right)$.

Next observe that $f_{r}(z, w)=\sum_{i} \llbracket \mathcal{N}_{i}\left(i_{r}(z, w)\right) \rrbracket$ has the property that each $\mathcal{N}_{i}\left(i_{r}(z, w)\right)$ is orthogonal to $T_{\boldsymbol{\Psi}\left(i_{r}(z, w)\right)} \mathcal{M}$. In particular, if $|z|=1$ and $r \downarrow 0$, the tangent planes $T_{\boldsymbol{\Psi}\left(i_{r}(z, w)\right)} \mathcal{M}$ converge to $\mathbb{R}^{2} \times\{0\}$ : it follows, by the uniform convergence of $f_{r}$ to $f_{0}$, that $f_{0}(z, w)=\sum_{i} \llbracket\left(f_{0}\right)_{i}(z, w) \rrbracket$ for some $\left(f_{0}\right)_{i}(z, w)$ which are orthogonal to $\mathbb{R}^{2} \times\{0\}$. We thus conclude that each $\left(f_{0}\right)_{i}(z, w)$ belongs to $\{0\} \times \mathbb{R}^{\bar{n}} \times\{0\}$. 
8.4. Step 4: Minimality of $g$. In order to complete the proof of Proposition 8.1 we need to show that $g$ is Dir-minimizing. Given the homogeneity of $g$ in the radial direction, it suffices to show that there is no $W^{1,2}$ multifunction $h: B_{1} \rightarrow \mathcal{A}_{Q}\left(\mathbb{R}^{\bar{n}}\right)$ which has the same trace of $g$ on $\partial B_{1}$ and less energy on $B_{1}$. Assume thus by contradiction that there is an $h \in W^{1,2}\left(B_{1}, \mathcal{A}_{Q}\left(\mathbb{R}^{\bar{n}}\right)\right)$ such that $\left.h\right|_{\partial B_{1}}$ and

$$
\int|D h|^{2} \leq \int|D g|^{2}-\delta
$$

for some positive $\delta>0$. Recall the definition of $W^{1,2}$ according to Remark 2.7: using the map $\mathbf{W}$ in there and the functions $h \circ \mathbf{W}$ and $g \circ \mathbf{W}$ we can use the theory of [4] and assume that $h \circ \mathbf{W}$ is a Dir-minimizer on the euclidean disk $D_{1} \subset \mathbb{R}^{2}$. Observe also that, since $\boldsymbol{\eta} \circ g \equiv 0$, we must have $\boldsymbol{\eta} \circ h \equiv 0$ as well. Indeed since $h \circ \mathbf{W}=g \circ \mathbf{W}$ on $\partial D_{1}$, we have $\boldsymbol{\eta} \circ h \circ \mathbf{W}=\boldsymbol{\eta} \circ g \circ \mathbf{W}=0$ on the boundary and considering that

$$
\int_{D_{1}} \sum_{i}\left|D\left(h_{i} \circ \mathbf{W}-\boldsymbol{\eta} \circ h \circ \mathbf{W}\right)\right|^{2} \leq \int_{D_{1}}|D(h \circ \mathbf{W})|^{2}-Q \int_{D_{1}}|D(\boldsymbol{\eta} \circ h \circ \mathbf{W})|^{2},
$$

the minimality of $h \circ \mathbf{W}$ forces the Dirichlet energy of $\boldsymbol{\eta} \circ h \circ \mathbf{W}$ to vanish identically.

Using (2.14), the decay $\mathbf{D}(r) \leq C r^{2 I_{0}}$ and a Fubini-type argument we can find a sequence of radii $s_{j} \rightarrow 0$ such that

$$
\int_{\partial B_{1}}\left|D f_{0}\right|^{2} \leq \limsup _{j} \int_{\partial B_{1}}\left|D f_{s_{j}}\right|^{2} \leq \limsup _{j} \frac{\mathbf{D}^{\prime}\left(s_{j}\right)}{s_{j}^{2 I_{0}-1}} \leq C .
$$

We now wish to "smooth" $h$, i.e. to approximate it with a sequence of Lipschitz maps $h_{\varepsilon}$ such that $\boldsymbol{\eta} \circ h_{\varepsilon} \equiv 0$,

$$
\begin{gathered}
\int_{B_{1}}\left|D h_{\varepsilon}\right|^{2}-|D h|^{2} \leq \varepsilon^{2} \\
\int_{\partial B_{1}} \mathcal{G}\left(f_{0}, h_{\varepsilon}\right)^{2}+\left.\left|\int_{\partial B_{1}}\right| D f_{0}\right|^{2}-\left|D h_{\varepsilon}\right|^{2} \mid \leq \varepsilon^{2} .
\end{gathered}
$$

We would like to appeal to [6, Lemma 3.5], but there is the slight technical complication that $\mathfrak{B}$ is not regular. We postpone this technical step and continue with the argument assuming the existence of the approximations $h_{\varepsilon}$.

Next we would like to apply [6, Lemma 3.6] to $h_{\varepsilon}$ and $\mathbf{p}_{T_{0} \Sigma}\left(f_{s_{j}}\right)=: \bar{f}_{s_{j}}$, to get a family of competitor functions $\left(\hat{f}_{s_{j}}\right) \subset W^{1,2}\left(B_{1}, \mathcal{A}_{Q}\left(\mathbb{R}^{2+\bar{n}}\right)\right)$, such that $\left.\left.\hat{f}_{s_{j}}\right|_{\partial B_{1}}=\left.\bar{f}_{s_{j}}\right|_{\partial B_{1}}\right)$ and

$$
\begin{gathered}
\int_{B_{1}}\left|D \hat{f}_{s_{j}}\right|^{2} \leq \int_{B_{1}}\left|D h_{\varepsilon}\right|^{2}+\varepsilon \int_{\partial B_{1}}\left(\left|D_{\tau} h_{\varepsilon}\right|^{2}+\left|D_{\tau} \bar{f}_{s_{j}}\right|^{2}\right)+\frac{C}{\varepsilon} \int_{\partial B_{1}} \mathcal{G}\left(h_{\varepsilon}, \bar{f}_{s_{j}}\right)^{2}, \\
\operatorname{Lip}\left(\hat{f}_{s_{j}}\right) \leq C\left(\operatorname{Lip}\left(h_{\varepsilon}\right)+\operatorname{Lip}\left(\bar{f}_{s_{j}}\right)+\frac{1}{\varepsilon} \sup _{\partial B_{1}} \mathcal{G}\left(\bar{f}_{s_{j}}, h_{\varepsilon}\right)\right) \\
\boldsymbol{\eta} \circ \hat{f}_{s_{j}}=\boldsymbol{\eta} \circ \bar{f}_{s_{j}} .
\end{gathered}
$$


Again, this is not straightforward because [6, Lemma 3.6] is stated for euclidean domains. We postpone this second technical problem and continue with our argument assuming the existence of $\hat{f}_{s_{j}}$.

We are now ready to define our competitor function. We set $\overline{\mathcal{L}}_{s_{j}}(z, w):=s_{j}^{I_{0}} \hat{f}_{s_{j}}\left(i_{1 / s_{j}}(z, w)\right)$ and, observing that $\overline{\mathcal{L}}_{s_{j}}$ takes value in $\mathcal{A}_{Q}\left(T_{0} \Sigma\right)$, we use (3.1) to define a corresponding $\mathcal{L}_{s_{j}}$, which clearly is a competitor $\mathcal{N}$ in $B_{s_{j}}$ according to Definition 3.1. Moreover

$$
\operatorname{Lip}\left(\mathcal{L}_{s_{j}}\right) \leq C s_{j}^{I_{0}+1} \operatorname{Lip}\left(\left.\hat{f}_{s_{j}}\right|_{B_{1}}\right) \stackrel{(8.6)}{\leq} C s_{j}{ }^{\eta}
$$

Therefore we can apply Proposition 3.2 with $\overline{\mathcal{L}}=\overline{\mathcal{L}}_{s_{j}}$. In particular, taking into account Theorem 7.3 and (8.12), we conclude that

$$
\int_{B_{s_{j}}}|D \overline{\mathcal{N}}|^{2} \leq\left(1+C s_{j}\right) \int_{B_{s_{j}}}\left|D \overline{\mathcal{L}}_{s_{j}}\right|^{2}+C \boldsymbol{m}_{0}^{1 / 2} \int_{B_{s_{j}}}|z|^{\gamma_{0}-1}\left|\boldsymbol{\eta} \circ \mathcal{L}_{s_{j}}\right|+C s_{j}^{2 I_{0}+\eta} .
$$

Next, recall the inequality (4.6):

$$
\int_{B_{s_{j}}}|z|^{\gamma_{0}-1}\left|\boldsymbol{\eta} \circ \mathcal{L}_{s_{j}}\right| \leq C \int_{B_{s_{j}}}|z|^{\gamma_{0}-1}\left|\boldsymbol{\eta} \circ \overline{\mathcal{L}}_{s_{j}}\right|+C \int_{B_{s_{j}}}|z|^{\gamma_{0}-1}\left|\overline{\mathcal{L}}_{s_{j}}\right|^{2} .
$$

By (8.17) the first term in the right hand side equals indeed

$$
C \int_{B_{s_{j}}}|z|^{\gamma_{0}-1}|\boldsymbol{\eta} \circ \overline{\mathcal{N}}| \leq C s_{j}^{\eta} \mathbf{D}\left(s_{j}\right) \leq C s_{j}^{2 I_{0}+\eta} .
$$

For the second term we use the Poincaré inequality

$$
\int_{B_{s_{j}}}|z|^{\gamma_{0}-1}\left|\overline{\mathcal{L}}_{s_{j}}\right|^{2} \leq C s_{j}^{1+\gamma_{0}} \int_{B_{s_{j}}}\left|D \overline{\mathcal{L}}_{s_{j}}\right|^{2}+C s_{j}^{\gamma_{0}} \int_{\partial B_{s_{j}}}\left|\overline{\mathcal{L}}_{s_{j}}\right|^{2}
$$

whose proof will be given in Lemma A.1.

Using that

$$
\int_{\partial B_{s_{j}}}\left|\overline{\mathcal{L}}_{s_{j}}\right|^{2}=\int_{\partial B_{s_{j}}}|\overline{\mathcal{N}}|^{2}=\mathbf{H}\left(s_{j}\right) \leq C s_{j}^{2 I_{0}+1}
$$

we easily conclude that

$$
\int_{B_{s_{j}}}|D \overline{\mathcal{N}}|^{2} \leq\left(1+C s_{j}\right) \int_{B_{s_{j}}}\left|D \overline{\mathcal{L}}_{s_{j}}\right|^{2}+C s_{j}^{2 I_{0}+\eta} .
$$

Changing variables and dividing by $s_{j}^{2 I_{0}}$ we infer that

$$
\int_{B_{1}}\left|D \bar{f}_{s_{j}}\right| \leq \int_{B_{1}}\left|D \hat{f}_{s_{j}}\right|^{2}+C s_{j}^{\eta} .
$$


Using (8.13), (8.14) and (8.15), we conclude

$$
\begin{aligned}
\int_{B_{1}}\left|D \bar{f}_{s_{j}}\right|^{2} & \leq \int_{B_{1}}|D h|^{2}+C s_{j}^{\eta}+C \varepsilon+\frac{C}{\varepsilon} \int_{\partial B_{1}} \mathcal{G}\left(f_{0}, \bar{f}_{s_{j}}\right)^{2} \\
& \leq \int_{B_{1}}|D g|^{2}-\delta+C s_{j}^{\eta}+C \varepsilon+\frac{C}{\varepsilon} \int_{\partial B_{1}} \mathcal{G}\left(f_{0}, \bar{f}_{s_{j}}\right)^{2},
\end{aligned}
$$

where the constant $C$ is independent of $\varepsilon$. In particular, if we fix $\varepsilon$ sufficiently small and we then let $s_{j} \downarrow 0$, by the uniform convergence of $f_{s_{j}}$ to $f_{0}$ on $\partial B_{1}$ we conclude

$$
\limsup _{j \rightarrow \infty} \int_{B_{1}}\left|D \bar{f}_{s_{j}}\right|^{2} \leq \int_{B_{1}}|D g|^{2}-\frac{\delta}{2} .
$$

Since however $f_{s_{j}} \rightarrow g$ in $B_{1}$, the latter inequality contradicts the semicontinuity of the Dirichlet energy.

8.5. Step 5: Technical leftovers. First of all we show the existence of the map $h_{\varepsilon}$ as in (8.13) and (8.14). We consider $h \circ \mathbf{W}$, which is defined on the closed unit disk $\bar{D}_{1} \subset \mathbb{R}^{2}$. We then can apply $\left[6\right.$, Lemma 3.5] to the latter map and generate approximations $\hat{h}_{\varepsilon}$ which satisfy the bounds (8.13) and (8.14) with $D_{1}$ in place of $B_{1}$ and $h \circ \mathbf{W}$ in place of $h$. The maps $h_{\varepsilon}:=\hat{h}_{\varepsilon} \circ \mathbf{W}$ would then satisfy the desired estimates because of the conformality of $\mathbf{W}^{-1}$ (which keeps the Dirichlet energy invariant) and its regularity in $B_{1} \backslash\{0\}$ (which results into the loss of a constant factor in (8.14)). However the resulting map would not be Lipschitz because of the singularity of $\mathbf{W}^{-1}$ in the origin. To overcome this difficulty it suffices to perturb slightly $\hat{h}_{\varepsilon}$ so that it is constant in a small neighborhood of the origin. As for the condition $\boldsymbol{\eta} \circ h_{\varepsilon} \equiv 0$, this can easily be achieved subtracting the average to whichever extension satisfies (8.13) and (8.14).

Secondly we show the existence of $\hat{f}_{s_{j}}$. First of all we observe that the condition (8.17) can be easily achieved after we prove the existence of a map which satisfies the other two conditions: as above it suffices to subtract the average of this map and add back $\boldsymbol{\eta} \circ \bar{f}_{s_{j}}$. At this point we observe that it suffices, as above, to compose with the map $\mathbf{W}$, apply [4, Lemma 2.14] and [5, Lemma 3.6] and compose the resulting map with $\mathbf{W}^{-1}$ : indeed the latter would coincide with $h_{\varepsilon} \circ \mathbf{W}$ on $D_{1-\varepsilon}$ and on the complement $\mathbf{W}^{-1}$ is regular.

\section{Appendix A. Some useful Lemmas.}

The first lemma is a simple version of the Poincaré inequality for $W^{1,2}$ functions.

Lemma A.1. There exists a universal constant $C>0$ such that the following two inequalities hold for every $f \in W^{1,2}\left(B_{r}, \mathcal{A}_{Q}\right)$ with $B_{r} \subset \mathfrak{B}_{Q}$ :

$$
\begin{array}{r}
\int_{B_{r}}|f|^{2} \leq C r^{2} \int_{B_{r}}|D f|^{2}+C r \int_{\partial B_{r}}|f|^{2} \\
\int_{B_{r}}|z|^{\gamma_{0}-1}|f|^{2} \leq C r^{1+\gamma_{0}} \int_{B_{r}}|D f|^{2}+C r^{\gamma_{0}} \int_{\partial B_{1}}|f|^{2} .
\end{array}
$$


Proof. By approximation we can assume, without loss of generality, that $f$ is Lipschitz and, by scaling, it suffices to show the inequalities (A.1) and (A.2) on the ball $B_{1}$. Fixing $|z|=1$ and integrating along rays

$$
\left|f\left(r z, r^{1 / Q} w\right)\right|^{2} \leq 2|f(z, w)|^{2}+2 \int_{r}^{1}\left|D f\left(t z, t^{1 / Q} w\right)\right|^{2} d t .
$$

Using radial coordinates we then conclude

$$
\int_{B_{1}}|z|^{\gamma_{0}-1}|f|^{2} \leq C \int_{\partial B_{1}}|f|^{2}+\int_{\partial B_{1}} \int_{0}^{1} r_{0}^{\gamma} \int_{r}^{1}\left|D f\left(t z, t^{1 / Q} w\right)\right|^{2} d t d r d z .
$$

Using Fubini the latter integral can be rewritten as

$$
\int_{0}^{1} \int_{\partial B_{1}}\left|D f\left(t z, t^{1 / Q} w\right)\right|^{2} \int_{0}^{t} r^{\gamma_{0}} d t d z d r \leq \int_{0}^{1} t \int_{\partial B_{1}}\left|D f\left(t z, t^{1 / Q} w\right)\right|^{2} d z d r .
$$

This completes the proof of (A.2). The proof of (A.1) is a simple variation of this one and is left to the reader.

Lemma A.2. Let $\overline{\mathcal{L}}: \mathfrak{B}_{\bar{Q}} \rightarrow \mathcal{A}_{Q}\left(\mathbb{R}^{2+\bar{n}}\right)$ be Lipschitz and consider the map $\mathcal{L}: \mathfrak{B}_{\bar{Q}} \rightarrow$ $\mathcal{A}_{Q}\left(\mathbb{R}^{2+n}\right)$ defined by (3.1). Then there exists a constant $C:=C\left(\left\|\Psi_{0}\right\|_{C^{3}}\right)>0$ such that

$$
\begin{gathered}
\mathcal{G}(\mathcal{L}, \overline{\mathcal{L}})(z, w) \leq C r|\overline{\mathcal{L}}|(z, w)+C|\overline{\mathcal{L}}|^{2}(z, w), \quad \forall(z, w) \in B_{r} \\
\int_{B_{r}}|D \mathcal{L}|^{2} \leq(1+C r) \int_{B_{r}}|D \overline{\mathcal{L}}|^{2}+C r \int_{\partial B_{r}}|\overline{\mathcal{L}}|^{2} .
\end{gathered}
$$

Proof. For what concerns (A.3), observe that $D \Psi(0)=0$ implies $\|D \Psi\|_{L^{\infty}\left(B_{r}\right)} \leq C r$. Therefore, by the $C^{3}$ regularity of $\Psi$, we get

$$
\begin{aligned}
\mathcal{G}(\mathcal{L}, \overline{\mathcal{L}})(z, w) & =\sum_{j=1}^{Q}\left|\Psi\left(\mathbf{p}_{0}(\boldsymbol{\Psi})+\overline{\mathcal{L}}_{j}\right)-\Psi\left(\mathbf{p}_{0}(\boldsymbol{\Psi})\right)\right|(z, w) \\
& \leq\|D \Psi\|(\mathbf{\Psi}(z, w))|\overline{\mathcal{L}}|(z, w)+\left\|A_{\Sigma}\right\||\overline{\mathcal{L}}|^{2}(z, w) \\
& \leq C r|\overline{\mathcal{L}}|(z, w)+C|\overline{\mathcal{L}}|^{2} .
\end{aligned}
$$

An analogous computation gives

$$
\int_{B_{r}}|D \mathcal{L}|^{2} \leq(1+C r) \int_{B_{r}}|D \overline{\mathcal{L}}|^{2}+C \int_{B_{r}}|\overline{\mathcal{L}}|^{2}
$$

and we conclude (A.4) using Lemma A.1.

\section{REFERENCES}

[1] Frederick J. Almgren, Jr. Almgren's big regularity paper, volume 1 of World Scientific Monograph Series in Mathematics. World Scientific Publishing Co. Inc., River Edge, NJ, 2000.

[2] Costante Bellettini and Tristan Rivière. The regularity of special Legendrian integral cycles. Ann. Sc. Norm. Super. Pisa Cl. Sci. (5), 11(1):61-142, 2012.

[3] Sheldon Xu-Dong Chang. Two-dimensional area minimizing integral currents are classical minimal surfaces. J. Amer. Math. Soc., 1(4):699-778, 1988. 
[4] Camillo De Lellis and Emanuele Spadaro. Q-valued functions revisited. Mem. Amer. Math. Soc., 211(991):vi+79, 2011.

[5] Camillo De Lellis and Emanuele Spadaro. Multiple valued functions and integral currents. Preprint, 2013.

[6] Camillo De Lellis and Emanuele Spadaro. Regularity of area-minimizing currents I: gradient $L^{p}$ estimates. Preprint, 2013.

[7] Camillo De Lellis and Emanuele Spadaro. Regularity of area-minimizing currents II: center manifold. Preprint, 2013.

[8] Camillo De Lellis and Emanuele Spadaro. Regularity of area-minimizing currents III: blow-up. Preprint, 2013.

[9] Camillo De Lellis, Emanuele Spadaro, and Luca Spolaor. Regularity theory for 2-dimensional almost minimal currents II: branched center manifold. 2015.

[10] Camillo De Lellis, Emanuele Spadaro, and Luca Spolaor. Regularity theory for 2-dimensional almost minimizing currents I: Lipschitz approximation. 2015.

[11] Camillo De Lellis, Emanuele Spadaro, and Luca Spolaor. Uniqueness of tangent cones for 2dimensional almost minimizing currents. 2015.

[12] David Pumberger and Tristan Rivière. Uniqueness of tangent cones for semicalibrated integral 2cycles. Duke Math. J., 152(3):441-480, 2010.

[13] Tristan Rivière and Gang Tian. The singular set of $J$-holomorphic maps into projective algebraic varieties. J. Reine Angew. Math., 570:47-87, 2004.

[14] Leon Simon. Lectures on geometric measure theory, volume 3 of Proceedings of the Centre for Mathematical Analysis, Australian National University. Australian National University Centre for Mathematical Analysis, Canberra, 1983.

[15] Brian White. Tangent cones to two-dimensional area-minimizing integral currents are unique. Duke Math. J., 50(1):143-160, 1983.

[16] Anton Zorich. Flat surfaces. In Frontiers in number theory, physics, and geometry. I, pages 437-583. Springer, Berlin, 2006. 